\title{
An Algorithm for the Construction of Parametrizing Bimodules for Homogeneous Modules Over Tubular Canonical Algebras
}

\author{
Piotr Dowbor · Hagen Meltzer • Andrzej Mróz
}

Received: 1 November 2012 / Accepted: 21 May 2013 / Published online: 27 June 2013

(c) The Author(s) 2013. This article is published with open access at SpringerLink.com

\begin{abstract}
We give an algorithmic description of matrix bimodules parametrizing all indecomposable homogeneous $\Lambda$-modules with a fixed slope $q$ over a tubular canonical algebra $\Lambda$, for all possible slopes $q$ (Main Theorem 3.3). A crucial role in this description is played by universal extensions of bimodules and their nice properties (Theorems 3.1 and 3.2).
\end{abstract}

Keywords Parametrizing bimodule $\cdot$ Homogeneous module Canonical tubular algebra • Universal extension • Derivation • Weighted projective line $\cdot$ Coherent sheaf $\cdot$ Algorithm

AMS 2010 Classifications $16 \mathrm{G} 20 \cdot 16 \mathrm{G} 60 \cdot 16 \mathrm{G} 70 \cdot 14 \mathrm{~L} 30 \cdot 15 \mathrm{~B} 99 \cdot 68 \mathrm{Q} 99$.

\section{Introduction}

The canonical algebras take a special position in contemporary representation theory of finite dimensional algebras over a field. They have been originally introduced and

Presented by Alain Verschoren

Piotr Dowbor and Andrzej Mróz are supported by the Research Grant N N201 542138 of the Polish Ministry of Science and Higher Education.

P. Dowbor $(\varangle)$ · A. Mróz

Faculty of Mathematics and Computer Science, Nicolaus Copernicus University, Chopina 12/18, 87-100 Toruń, Poland

e-mail: dowbor@mat.uni.torun.pl

A. Mróz

e-mail: amroz@mat.uni.torun.pl

H. Meltzer

Institute of Mathematics, Szczecin University, Wielkopolska 15, 70-451 Szczecin, Poland e-mail: meltzer@wmf.univ.szczecin.pl 
intensively studied by Ringel in [19], who determined precisely their representation types and described the structure of their module categories. An important role in a better understanding of the module categories for canonical algebras has been played by the approach via coherent sheaves over the so called weighted projective lines, due to Geigle and Lenzing [11]. It has especially brought interesting effects in the case of tubular canonical algebras, which yield a model example of tame algebras having non-domestic, but polynomial growth representation type.

At present, the structure of the category $\bmod \Lambda$ of finite dimension right $\Lambda$ modules for a tubular canonical $k$-algebra $\Lambda=\Lambda(\mathbf{p}, \lambda)$ is completely understood and described. In particular, full information on the shape of the Auslander-Reiten quiver $\Gamma_{\Lambda}$ is available, with a precise description of its connected components: the preprojective and preinjective one, as well as the collection of $\mathbb{P}^{1}(k)$-families $\widetilde{\mathcal{T}}^{(q)}$ of tubes, indexed by the set $\overline{\mathbb{Q}}_{0}^{\infty}:=\mathbb{Q}_{\geq 0} \cup\{\infty\}$, alternatively $\overline{\mathbb{Q}}^{\prime}:=\mathbb{Q}_{\leq 0} \cup \mathbb{Q}_{\geq p} \cup\{\infty\}$, if we use for the "separation process" the slope instead of the index function ( $p$ denotes the greatest common divisor of the numbers $p_{1}, \ldots, p_{t}$ forming the weight sequence $\mathbf{p}$ ). In result, the classification of all indecomposable $\Lambda$-modules, up to isomorphism, referring to their dimension vectors and "tubular coordinates" is known since many years. Nevertheless, a complete list of matrix presentations representing all isomorphism classes of indecomposable $\Lambda$-modules is not known up to now and its creation remains one of the very last important open questions concerning representation theory of tubular canonical algebras. Notice, that as far as now there was even no possibility of an effective direct verification of the definition of tameness for $\Lambda$ (see e.g. [18], cf. also Section 2.1). On the other hand the existence of such a list would be very useful in studying other challenging open problems of representation theory for $\Lambda$ like determining the multiplicity vectors or isomorphism questions (see [5-7]).

In the last years a remarkable progress in matrix description of indecomposable modules over tubular canonical algebras $\Lambda$ has been achieved. In particular, a certain nontrivial effective algorithm for creating matrix presentations for indecomposables from the exceptional tubes was discovered (see [8], also [17]). There were obtained also some results [13], which allow to find matrix presentations of preprojective and preinjective indecomposables over the corresponding hereditary algebra of Euclidean type using tilting theory. Recently in [9], also the full description of matrix presentations for all the indecomposable $\Lambda$-modules from homogeneous tubes of integral slopes was presented, which is especially important in the context of this paper.

This article is in a natural way a completion of [9]. We study here the problem of constructing matrix presentations for indecomposable $\Lambda$-modules from all homogeneous tubes in the remaining (non integral) slopes. We give an effective recursive algorithmic construction, creating for each slope $q \in \overline{\mathbb{Q}}^{\prime}$ a matrix bimodule $k[\xi]_{f} B_{\Lambda}\left(={ }_{k[\xi]_{f}} B_{\Lambda}(q)\right)$, which yields a parametrization (by means of a tensor product) of all indecomposable $\Lambda$-modules in homogeneous tubes from the $\mathbb{P}^{1}(k)$-family $\tilde{\mathcal{T}}^{(q)}$ by indecomposable $k[\xi]_{f}$-modules, where $k[\xi]_{f}$ is the localization of the polynomial algebra $k[\xi]$ in one variable $\xi$, defined by the polynomial $f=f_{\mathbf{p}} \in k[\xi]$. The bottom of this recursion is the list of the bimodules $B(q):=B^{(q)}$, for $q \in \overline{\mathbb{Q}}^{\prime} \cap \mathbb{Z}$ given in [9, Table 4.7]. Roughly speaking, with any $q \in \mathbb{Q}_{\geq p}$ (respectively $q \in \mathbb{Q}_{\geq 7}$, if $\mathbf{p}=$ $(2,3,6))$ we associate a certain uniquely determined sequence $q_{0}=q, q_{1}, \ldots, q_{s} \in$ $\mathbb{Q}_{\geq 0}$, with $q_{s} \in \mathbb{Z}$, which can be easily computed by means of the Euclidean algorithm 
(similarity to the chain fraction decomposition). Next we reconstruct successively the bimodules $B\left(q_{s-1}\right), \ldots, B\left(q_{1}\right), B\left(q_{0}\right)$ from $B\left(q_{s}\right):=B^{\left(q_{s}\right)}$ by applying an effective, from the computational point of view, construction of universal extension for bimodules (cf. Proposition 5.2 and Theorem 5.2; the case $q \in \mathbb{Q}_{\leq 0}$ follows easily from the previous one). We are even able to give formulas, which in fact reduce the matrix description of the bimodules $B(q)$ to linear algebra computations over $k[\xi]_{f}$ (see the bimodules $B^{(q)}$ in Main Theorem 3.3(a), cf. Proposition 5.3). Recall that once we know the shape of the matrix bimodule $B(q)$, then for any pair $(a, l) \in(k \backslash \mathcal{Z}(f)) \times \mathbb{N}_{+}$, replacing fractions $\frac{g}{h} \in k[\xi]_{f}$ in the $k[\xi]_{f}$-matrices forming $B(q)$ by the matrices $g\left(J_{l}(a)\right) \cdot h\left(J_{l}(a)\right)^{-1} \in \mathbb{M}_{l \times l}(k)$, where $J_{l}(a)$ is the $l \times l$-Jordan block with the eigenvalue $a \in k_{f}$, we obtain the matrix $\Lambda$-module representing the indecomposable homogeneous module $\mathcal{H}_{q}(a, l)$ with slope $q$ and quasi-length $l$, over the point $a \in \mathbb{P}^{1}(k)$ (see Section 2.1 for details).

Observe that this result closes definitely the fundamental problem of the positive verification of tameness for a tubular canonical algebra $\Lambda$.

Our algorithmic construction uses mainly the technique of universal extensions of bimodules (see Main Theorem 3.3). Therefore in Section 4 we discuss general properties of this notion, which are necessary in further consideration. We prove the existence theorem (Theorem 4.1) and first of all the important result on the commutativity of universal extensions with tensor products appearing in the definition of tame algebra (Theorem 3.1). For this aim we discuss the behaviour of universal extensions of bimodules with respect to extension and restriction of the coefficient ring (Theorems 4.4 and 4.6, respectively; their proofs in some moments require a detail analysis of various natural isomorphisms), and next prove some rather delicate result which shows a real meaning of stability of the "Ext-dimension sequence" appearing in assumptions of Theorem 3.1 (Proposition 4.11). Finally, we prove Theorem 3.2 being the heart of our algorithm and describing constructively elementary changes of parametrizing bimodules together with their control on the slope level. In the proof of this last result we apply the equivalence $\mathcal{D}^{b}(\bmod \Lambda) \simeq$ $\mathcal{D}^{b}(\operatorname{coh} \mathbb{X})$ of the bounded derived categories, where coh $\mathbb{X}$ is the category of graded coherent sheaves over the weighted projective line $\mathbb{X}$ associated to $\Lambda$, and moreover, the technique of tubular mutations (see $[11,15,16]$ ).

Section 5 is fully devoted to the proof of Theorem 3.3. Its most important part deals with the "slope combinatorics", which yields a basis of our recursive procedure on the slope level (Proposition 5.2) and next allows to formulate the closed formulas for all the intermediate slopes appearing when the recursion is executed (Proposition 5.3, cf. also Main Theorem 3.3(a)). As a consequence of Theorem 3.2 we obtain first Theorem 5.2, which is a counterpart of Proposition 5.2 on the module level and in fact describes already fully our recursion. Then Theorem 5.2 together with Proposition 5.3 give the proof of the "general part" (a) of Main Theorem 3.3, which covers all the cases $(\mathbf{p}, q)$ except the case $6<q<7$, for the weight sequence $\mathbf{p}=(2,3,6)$. The assertion (b), dealing with the exceptional case, is a kind of "patch", which fills in the gap resulting of the fact that there exists no stable tube of maximal rank in $\widetilde{\mathcal{T}}^{(p)}$, for $\mathbf{p}=(2,3,6)$. To prove it we apply nice properties of the kernel construction and proceed analogously as in the universal extension part. In particular, we prove Theorem 5.4 on the commutativity of kernels with tensor products, using Proposition 5.5 explaining the role of stability of the "Hom-dimension sequence", and next we combine this result with certain information on the "slope combinatorics" and the arguments 
similar to Theorem 3.2. We finish the paper with a concrete example showing that the presented procedure is really effective and allows to compute matrix bimodules parametrizing all homogeneous modules in a fixed slope over tubular canonical algebras.

Let us clearly stress that the proposed algorithmic procedure for creating parametrizing matrix bimodules is fully effective from the computational point of view in the sense that in each of its steps one can provide concrete calculations; in particular, linear algebra calculations over $k[\xi]$ realizing the applied bimodule constructions. In result it can be converted into a computer program and implemented e.g. as a MAPLE or GAP routine package.

In the paper we use standard definitions and notation which are well known and commonly used. For example, we denote by $\mathbb{N}\left(\right.$ resp. $\left.\mathbb{N}_{+}, \mathbb{N}_{\geq m}\right)$ the set of all natural numbers with 0 (resp. positive natural numbers, natural numbers greater or equal $m$ ). We use the similar notation in case of the sets $\mathbb{Z}$ and $\mathbb{Q}$ consisting of all integers and rational numbers, respectively. Moreover, for $n \in \mathbb{N}_{+}$we set $[n]:=\{1, \ldots, n\}$. However for the benefit of the reader, we briefly recall the most important basic notions in the consecutive sections. For other information concerning modules and representation theory of algebras (respectively, derived categories, matrix calculus) we refer to [1] and [2] (respectively, [12] and [10]).

A field $k$ is usually assumed to be algebraically closed. For any polynomial $f \in$ $k[\xi]$ in one variable $\xi$ its zero set is always denoted by $\mathcal{Z}(f)$.

\section{Preliminaries}

Let $k$ be as above. For a $k$-algebra $\Lambda$, we denote by MOD $\Lambda$ the category of right $\Lambda$ modules and by mod $\Lambda$ the full subcategory formed by all finite dimensional objects of MOD $\Lambda$. We start by briefly recalling the notion of tameness for bound quiver algebras, in particular, the role of parametrizing bimodules.

Let $\Lambda=k Q / I$ be a (finite dimensional) $k$-algebra of a bound quiver $(Q, I)$ and $R$ a commutative $k$-algebra. Then $R \otimes_{k} \Lambda$ is an $R$-algebra and the category MOD $R \otimes_{k} \Lambda$ of all right $R \otimes_{k} \Lambda$-modules, in fact $R$ - $\Lambda$-bimodules, can be viewed as category of all representations $B=\left(\left\{B_{x}\right\}_{x \in Q_{0}},\left\{B_{\alpha}: B_{t(\alpha)} \rightarrow B_{s(\alpha)}\right\}_{\alpha \in Q_{1}}\right)$ of $\left(Q^{\text {op }}, I^{\text {op }}\right)$ in the category MOD $R$, where $Q_{0}$ and $Q_{1}$ denote the sets of vertices and arrows of $Q$, respectively; whereas $s(\alpha)$ and $t(\alpha)$ denote the source and the terminus of an arrow $\alpha \in Q_{1}$. (Clearly, each $B$ is an $R$-module and a $\Lambda$-module, we denote the restricted structures on $B$ by ${ }_{R} B$ and $B_{\Lambda}$, respectively). By $\bmod _{R} \Lambda$ we denote the full subcategory of MOD $R \otimes_{k} \Lambda$ formed by all $B$ such that all $R$-modules $B_{x}$ are finitely generated free, for $x \in Q_{0}$, and by $\bmod _{R}^{\text {mat }} \Lambda$ its dense subcategory formed by all $B$ such that $B_{x}=R^{m_{x}}$ for every $x \in Q_{0}$, where $m_{x} \in \mathbb{N}$ (then $B_{\alpha}$ can be uniquely regarded as matrices from $\mathbb{M}_{m_{s(\alpha)} \times m_{t(\alpha)}}(R)$ and therefore we often call $B$ a matrix bimodule). Clearly, if $R=k$ then the isomorphism $R \otimes_{k} \Lambda \cong \Lambda$ yields the identifications $\operatorname{MOD} R \otimes_{k} \Lambda=\operatorname{MOD} \Lambda$ and $\bmod _{R} \Lambda=\bmod \Lambda$; in particular, the dense subcategory $\bmod ^{\mathrm{mat}} \Lambda:=\bmod _{R}^{\mathrm{mat}} \Lambda$ of $\bmod \Lambda$ consists of all matrix representations of $\Lambda$. For $B$ in $\bmod _{R} \Lambda$, by rank vector of $B$ we mean the vector $\underline{\mathrm{rk}} B=\left(\operatorname{rk}_{R}\left(B_{x}\right)\right) \in \mathbb{N} Q_{0}$, where $\operatorname{rk}_{R}\left(B_{x}\right)$ denotes the rank of a free $R$-module $B_{x}$, for every $x \in Q_{0}$. 
Let $B=\left(\left\{B_{x}\right\}_{x \in Q_{0}},\left\{B_{\alpha}\right\}_{\alpha \in Q_{1}}\right)$ be an $R \otimes_{k} \Lambda$-module. Then for any $R$-module $N$ we have an $R \otimes_{k} \Lambda$-module (so in particular a $\Lambda$-module) $N \otimes_{R} B=(\{N \otimes$ $\left.{ }_{R} B_{x}\right\}_{x \in Q_{0}},\left\{\operatorname{id}_{N} \otimes B_{\alpha}\right\}_{\alpha \in Q_{1}}$ ). If $R=k[\xi]_{f}$, where $k[\xi]_{f}$ is the localization of the polynomial algebra $k[\xi]$ with respect to the multiplicative system defined by a nonzero polynomial $f \in k[\xi]$, then for any $a \in k_{f}$ and $l \in \mathbb{N}_{+}$, by $B(a, l)$ and $B(a)$, we mean the $\Lambda$-modules $\left(R /(\xi-a)^{l} \otimes_{R} B\right)_{\Lambda}$ and $\left(R /(\xi-a) \otimes_{R} B\right)_{\Lambda}$, respectively, where $k_{f}=$ $k \backslash \mathcal{Z}(f)$. Note that if $B$ belongs to $\bmod _{R}^{\text {mat }} \Lambda$ then for any pair $(a, l) \in(k \backslash \mathcal{Z}(f)) \times$ $\mathbb{N}_{+}$, the $\Lambda$-module $B(a, l)$ can be regarded as a matrix module with matrices $B(a, l)_{\alpha} \in$ $\mathbb{M}_{l m_{s(\alpha)} \times \operatorname{lm}_{t(\alpha)}}(k)$, obtained from the matrices $B_{\alpha}=\left[g_{i, j}^{(\alpha)}\right] \in \mathbb{M}_{m_{s(\alpha)} \times m_{t(\alpha)}}(R)$ forming $B$, by the formula $B(a, l)_{\alpha}=\left[g_{i, j}^{(\alpha)}\left(J_{l}(a)\right)\right]$.

If $R$ is as above then for any $k[\xi] \otimes_{k} \Lambda$-module $\dot{B}$, we denote by $\dot{B}_{f}$ the $R \otimes_{k} \Lambda$ module $\dot{B}_{f}:=R \otimes_{k[\xi]} \dot{B}$. Clearly, if $\dot{B}$ belongs to $\bmod _{k[\xi]}^{\text {mat }} \Lambda$ then so does $\dot{B}_{f}$; in fact, $\dot{B}_{f}$ is given by the same matrices as $\dot{B}$ but presently regarded as matrices over $R$. Moreover, for any pair $(a, l)$ as above we always have the canonical $\Lambda$-isomorphism $\dot{B}_{f}(a, l) \cong \dot{B}(a, l)$.

Using the notions and notations introduced above, we formulate below a variant of Crawley-Boevey's characterization of tameness for algebras $\Lambda=k Q / I$ (see [4], cf. also [9]). This characterization is given in terms of bimodules, which yield parametrizations of all $\Lambda$-modules belonging to the whole family of homogeneous tubes in the Auslander-Reiten quiver of $\Lambda$, simultaneously in all dimension vectors being multiplicities of the rank vector of these bimodules, by indecomposable modules over affine localizations of $k[\xi]$.

For a pair $d, d^{\prime} \in \mathbb{N} Q_{0}$ of vectors we say that $d$ divides $d^{\prime}$ (and write $d \mid d^{\prime}$ ), if $d^{\prime}=l \cdot d$, for some $l \in \mathbb{N}$; then the unique such $l$ is denoted by $\frac{d^{\prime}}{d}$.

Theorem $A$ k-algebra $\Lambda$, as above, has tame representation type if and only if there exists a family $B^{(v)}, v \in \Omega$, of $A_{v}$ - $\Lambda$-bimodules in $\bmod _{A_{v}} \Lambda$ with the rank vectors $r^{(v)}:=\underline{\mathrm{rk}} B^{(v)} \in \mathbb{N}^{Q_{0}}$, where $A_{v}:=k[\xi]_{f_{v}}$ for some $f_{v} \in k[\xi]$, satisfying the following two conditions:

- $\quad$ the functors $-\otimes_{A_{v}} B^{(v)}: \bmod A_{v} \rightarrow \bmod \Lambda$, for $v \in \Omega$, induce an injection between the sets of isoclasses of indecomposable modules and preserve AuslanderReiten sequences,

- for every $d \in \mathbb{N}^{Q_{0}}$, the set $\Omega(d):=\left\{v \in \Omega: r^{(v)} \mid d\right\}$ is finite and has the property that for a subset $\Omega^{\prime} \subseteq \Omega(d)$, the union

$$
\bigcup_{v \in \Omega^{\prime}}\left\{B^{(v)}\left(a, \frac{d}{r^{(v)}}\right): a \in k \backslash \mathcal{Z}\left(f_{v}\right)\right\}
$$

meets all but finitely many isoclasses of indecomposable $\Lambda$-modules of dimension vector $d$ if and only if $\Omega^{\prime}=\Omega(d)$.

(Note that $\Omega=\bigcup_{d \in \mathbb{N} Q_{0}} \Omega(d)$ and $\Omega(d) \subseteq \Omega\left(d^{\prime}\right)$, if $d \mid d^{\prime}$ ).

The bimodules $B^{(v)}, v \in \Omega$, satisfying the conditions above are called parametrizing bimodules for all indecomposable $\Lambda$-modules from the family of homogeneous tubes. (Observe that for a fixed $v$, the modules $B^{(v)}(a, l)$, for $(a, l) \in k \backslash \mathcal{Z}\left(f_{v}\right) \times \mathbb{N}_{+}$, form a 1-parameter family of homogeneous tubes in $\bmod \Lambda$ consisting of modules with dimension vectors in $\left.\mathbb{N}_{+} \cdot r^{(v)}\right)$. 
By a canonical algebra we mean a canonical algebra of quiver type over $k$ in the sense of [19]. Such an algebra is given, up to isomorphism, as a quotient $k Q_{\mathbf{p}} / I_{\mathbf{p}, \lambda}$, where $Q_{\mathbf{p}}$ is the quiver
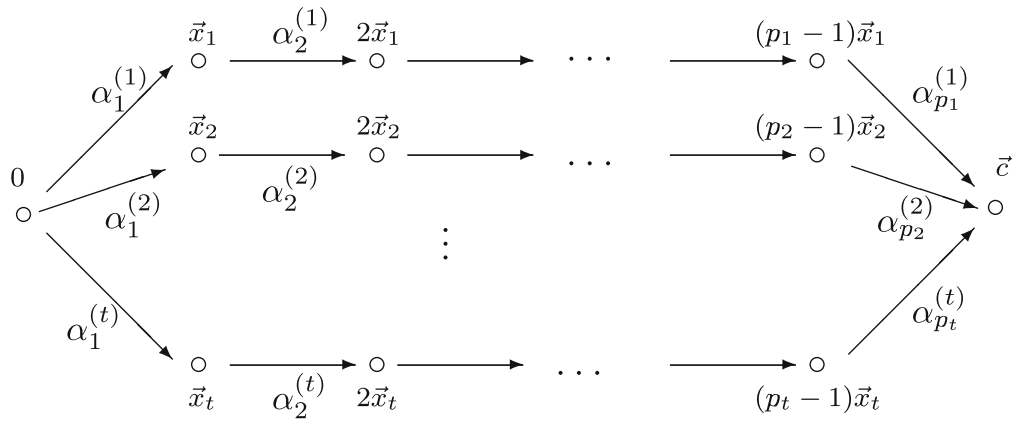

and $I_{\mathbf{p}, \lambda}$ is the ideal in the path algebra $k Q_{\mathbf{p}}$ generated by all elements $\alpha_{p_{i}}^{(i)} \cdots \alpha_{2}^{(i)}$ $\alpha_{1}^{(i)}-\alpha_{p_{1}}^{(1)} \cdots \alpha_{2}^{(1)} \alpha_{1}^{(1)}-\lambda_{i} \alpha_{p_{2}}^{(2)} \cdots \alpha_{2}^{(2)} \alpha_{1}^{(2)}, i=3, \ldots, t$, where the $\lambda_{i}$ are pairwise distinct non-zero elements of $k$. Thus $\Lambda$ depends on a sequence of natural numbers $\mathbf{p}=$ $\left(p_{1}, p_{2}, \ldots, p_{t}\right)$ and a sequence of parameters $\lambda=\left(\lambda_{3}, \ldots, \lambda_{t}\right)$. Recall that $\Lambda=$ $\Lambda(\mathbf{p}, \lambda)$ is called a tubular canonical algebra if and only if the sequence $\mathbf{p}$ is, up to permutation, one of the following $(2,2,2,2),(3,3,3),(2,4,4)$ or $(2,3,6)$. Without loss of generality one can assume that $\lambda_{3}=1$ and then, in case $t=3$, we simply write $\Lambda(\mathbf{p})$ assuming that $p_{1} \leq p_{2} \leq p_{3}$. A tubular canonical algebra $\Lambda$ of type $\mathbf{p}=(2,2,2,2)$ depends on one parameter $\lambda=\lambda_{4}$ and dependently on the context we write $\Lambda((2,2,2,2))$ or $\Lambda((2,2,2,2), \lambda)$.

We denote by $\Lambda_{0}$ (respectively, $\Lambda_{\infty} \cong \Lambda_{0}^{o p}$ ) the hereditary algebra which is obtained from $k Q_{\mathbf{p}}$ by deleting the vertex $\vec{c}$ (respectively, 0 ).

Recall that any finite-dimensional $\Lambda$-module $M$ (i.e. an object of $\bmod \Lambda$ ) is described by finite-dimensional vector spaces $M_{i}$ for each vertex $i$ of $Q_{0}$ and by linear maps $M_{\alpha}: M_{j} \rightarrow M_{i}$ for each arrow $\alpha: i \rightarrow j$ of $Q_{1}$ such that the relations of the ideal $I$ are satisfied by the linear maps, where $Q_{\mathbf{p}}=\left(Q_{0}, Q_{1}\right)$. The collections $M=\left(\left\{M_{i}\right\}_{i \in Q_{0}},\left\{M_{\alpha}\right\}_{\alpha \in Q_{1}}\right)$ as above are usually called representations of $\Lambda$. For $M$ as above we always denote by $\underline{\operatorname{dim}}(M)$ the vector $\left(\operatorname{dim}_{k}\left(M_{i}\right)\right)_{i \in Q_{0}} \in \mathbb{N}^{Q_{0}}$, called the dimension vector of $M$. To $M$ we can associate also two integers, its rank and degree, given by the formulas

$$
\begin{gathered}
\operatorname{rk}(M):=\operatorname{dim}_{k} M_{0}-\operatorname{dim}_{k} M_{\vec{c}} \\
\operatorname{deg}(M):=\sum_{i=1}^{t} \frac{p}{p_{i}}\left(\sum_{j=1}^{p_{i}-1} \operatorname{dim}_{k} M_{j \vec{x}_{i}}\right)-p \cdot \operatorname{dim}_{k} M_{\vec{c}}
\end{gathered}
$$

where $p$ denotes the least common multiple of the numbers $p_{1}, \ldots, p_{t}$ (note that $p=$ $p_{t}$, if $\mathbf{p}$ is of tubular type). They give rise to two linear forms rk, deg $: K_{0}(\Lambda) \rightarrow \mathbb{Z}$. For an indecomposable $\Lambda$-module $M$ we define also its slope $\mu(M):=\frac{\operatorname{deg}(M)}{\operatorname{rk}(M)} \in \overline{\mathbb{Q}}$, where $\overline{\mathbb{Q}}=\mathbb{Q} \cup\{\infty\}$. (Clearly, the value of $\mu(M)$ depends only on $\underline{\operatorname{dim}}(M)$ ). 
For any $q \in \overline{\mathbb{Q}}$, we denote by $\mathcal{X}_{q}$ the full subcategory of $\bmod \Lambda$ formed by all indecomposable $\Lambda$-modules with $\mu(M)=q$.

Let $\Lambda$ be a tubular canonical algebra. Then the structure of the Auslander-Reiten quiver $\Gamma_{\Lambda}$ of $\bmod \Lambda$, in particular the shape of its components, is well known [19]. Replacing by the slope the notion of index used originally by Ringel, it can be roughly described as follows:

$$
\Gamma_{\Lambda}=\mathcal{P} \sqcup\left(\coprod_{q \in \overline{\mathbb{Q}}^{\prime}} \widetilde{\mathcal{T}}^{(q)}\right) \sqcup \mathcal{Q}
$$

where $\overline{\mathbb{Q}}^{\prime}:=\mathbb{Q}_{\leq 0} \cup \mathbb{Q}_{\geq p} \cup\{\infty\}$ and

- $\quad \mathcal{P}$ is a preprojective component which coincides with the preprojective component of $\bmod \Lambda_{0}$,

- $\quad \widetilde{\mathcal{T}}=\left(\widetilde{\mathcal{T}}^{(q)}\right)_{q \in \overline{\mathbb{Q}}^{\prime}}$ consists of so-called separating $\mathbb{P}^{1}(k)$-families of tubes, all but $\widetilde{\mathcal{T}}^{(p)}$ and $\widetilde{\mathcal{T}}^{(0)}$ consisting only of stable ones (i.e. not containing a projective or injective module) of type $\mathbf{p}$,

- $\mathcal{Q}$ is a preinjective component which coincides with the preinjective component of $\bmod \Lambda_{\infty}$.

Moreover, for a fixed $q \in \overline{\mathbb{Q}}^{\prime}$ all dimension vectors of the $\Lambda$-modules from the tubes in the family $\widetilde{\mathcal{T}}^{(q)}$ have slope $q$ and $\mathcal{X}_{q}$ coincides with the full subcategory $<\widetilde{\mathcal{T}}^{(q)}>$ of $\bmod \Lambda$ formed by all indecomposable modules isomorphic to those belonging to the family $\widetilde{\mathcal{T}}^{(q)}$. (For simplicity we also denote by $\mathcal{X}_{q}^{0}$ the full subcategory of $\mathcal{X}_{q}$ formed by all homogeneous modules, i.e. the modules lying in all homogeneous tubes from $\left.\widetilde{\mathcal{T}}^{(q)}\right)$.

Recall that there exists an elementary bijection $\overline{\mathbb{Q}}^{\prime} \longleftrightarrow \overline{\mathbb{Q}}_{0}^{\infty}:=\mathbb{Q}_{\geq 0} \cup\{\infty\}$, which allows easily to translate slope to index, and conversely (see [9, 2.2]).

This very good knowledge of the structure of the category $\bmod \Lambda$ uniquely indicates that $\Lambda$ should have tame representation type, however, the precise description of all the bimodules $B^{(v)}, v \in \Omega$, parametrizing the indecomposables in all homogeneous tubes is not known (cf. Section 2.1). It is clear that $\Omega$ has to be equal to $\overline{\mathbb{Q}}^{\prime}$. Moreover, we can determine the rank vectors $r^{(q)}$ of $B^{(q)}$, for $q \in \overline{\mathbb{Q}}^{\prime}$. For any $d \in \mathbb{N}^{Q_{0}}$ we have $\Omega(d)=\left\{B^{(q)}\right\}$, if $d \in \mathbb{N}_{+} \cdot r^{(q)}$ for some $q$; otherwise, $\Omega(d)=\emptyset$.

In [9] we gave precise formulas for the bimodules $B^{(q)}$, for all $q \in \overline{\mathbb{Q}}^{\prime} \cap \mathbb{Z}$. Applying this result in this paper we construct a recursive algorithm for determining the bimodules $B^{(q)}$ for the remaining $q \in \overline{\mathbb{Q}}^{\prime}$. (The case $\overline{\mathbb{Q}}^{\prime} \cap \mathbb{Z}$ is just the bottom of the recursion.)

\section{Main Results}

\section{1}

In the construction of the algorithm we use the technique of universal extensions for bimodules. Our first result discuss the commutativity of universal extensions with tensor products appearing in the definition of tame algebra. 
For any $B$ in $\bmod _{R} \Lambda$, we denote by $\Upsilon_{\mathcal{F}}(B)$ the $R \otimes_{k} \Lambda$-module being the middle term of the universal (in fact couniversal) extension $\eta_{B}^{\mathcal{F}}$ of $B$ with respect to a fixed finite $R$-semisimple subcategory $\mathcal{F}$ of MOD $R \otimes_{k} \Lambda$ (if it exists, for the precise definitions and more details see Section 4.1, also [14, 16]).

Theorem Let $\Lambda=k Q /$ I be a finite dimensional $k$-algebra of a bound quiver $(Q, I)$, $R=k[\xi]_{f}$ the localization of $k[\xi]$, where $f \in k[\xi]$ is a nonzero polynomial, and $\mathcal{F}:=\left\{F_{1}, \ldots, F_{\rho}\right\}$ a semisimple subcategory in $\bmod \Lambda$. Then the $R$-category $\tilde{\mathcal{F}}:=$ $\left\{R \otimes_{k} F_{1}, \ldots, R \otimes_{k} F_{\rho}\right\}$ is $R$-semisimple; moreover, for any $R \otimes_{k} \Lambda$-module $B$ from $\bmod _{R} \Lambda$ such that the dimension sequence

$$
\left(\operatorname{dim}_{k} \operatorname{Ext}_{\Lambda}^{1}\left(B(a), F_{i}\right)\right)_{a \in k_{f}}
$$

is constant for every $i=1, \ldots, \rho$, the universal extension $\eta_{B}^{\widetilde{\mathcal{F}}}$ of $B$ with respect to $\widetilde{\mathcal{F}}$ in $\bmod _{R} \Lambda$ exists and

$$
\left(\Upsilon_{\mathcal{F}}(B)\right)(a, l) \cong \Upsilon_{\mathcal{F}}(B(a, l))
$$

in $\bmod \Lambda$, for all $a \in k_{f}$ and $l \in \mathbb{N}_{+}$.

The proof of this result needs deeper information on the behaviour of universal extensions for $R \otimes_{k} \Lambda$-modules with respect to extension and restriction of the coefficient ring. It will be given in Section 4.11.

Our next result plays an absolutely fundamental role in the construction of the algorithm creating the parametrizing bimodules for families of homogeneous tubes for tubular canonical algebras, proposed in the paper.

Theorem Let $\Lambda$ be a tubular canonical algebra of the tubular type $\mathbf{p}=\left(p_{1}, \ldots, p_{t}\right)$. Assume that a $k[\xi]_{f} \otimes_{k} \Lambda$-module $B$ in $\bmod _{k[\xi]_{f}} \Lambda$, with $f=f_{\mathbf{p}}$, is a parametrizing bimodule for the whole family of homogeneous tubes in $\mathcal{X}_{\mu^{\prime}}$, for $\mu^{\prime} \in \mathbb{Q}, \mu^{\prime}>p$, where

$$
f_{\mathbf{p}}:=\left\{\begin{array}{lll}
\xi(\xi-1)(\xi-\lambda) & \text { if } \quad \mathbf{p}=(2,2,2,2) \\
\xi(\xi-1) & \text { if } \quad \mathbf{p} \neq(2,2,2,2)
\end{array}\right.
$$

Then for any $\mu \in \mathbb{Q}$ such that $\mu^{\prime}>\mu \geq p$, and any stable tube $\mathcal{T}$ in $\mathcal{X}_{\mu}$, the universal extension of $B$ with respect to the $k[\xi]_{f}$-category $\mathcal{F}=\left\{k[\xi]_{f} \otimes_{k} F_{1}, \ldots, k[\xi]_{f} \otimes_{k} F_{\rho}\right\}$, for the semisimple category $\mathcal{F}=\mathcal{F}(\mathcal{T})$ formed by all quasi-simple objects $F_{i}:=$ $\tau^{i-1}\left(F_{1}\right)$ in $\mathcal{T}$, for $i=1, \ldots, \rho$, where $\rho=\rho_{\mathcal{T}}$ is the rank of $\mathcal{T}$, exists. Moreover, the $k[\xi]_{f} \otimes_{k} \Lambda$-module $\Upsilon_{\widetilde{\mathcal{F}}}(B)$ is a parametrizing bimodule for the whole family of homogeneous tubes in $\mathcal{X}_{\mu^{\prime \prime}}$, where $\mu^{\prime \prime}=\mu^{\prime \prime}\left(\mu, \mu^{\prime} ; m\right)>p$ is given by the formula

$$
\mu^{\prime \prime}=\frac{d m\left(r d^{\prime}-r^{\prime} d\right)+d^{\prime}}{r m\left(r d^{\prime}-r^{\prime} d\right)+r^{\prime}}
$$

for $d, r, d^{\prime} r^{\prime} \in \mathbb{N}$ such that $\mu=\frac{d}{r}, \mu^{\prime}=\frac{d^{\prime}}{r^{\prime}}$ are irreducible fraction presentations, and $m=m_{\mathcal{T}}:=\frac{p}{\rho_{\mathcal{T}}}$. 
The proof of this theorem heavily depends on Theorem 3.1 and it will be presented in Section 4.12.

\section{3}

To formulate our main result, being a description of the announced algorithm, we have to fix some extra notation.

Let $\Lambda$ be a canonical algebra of the type $\mathbf{p}=\left(p_{1}, \ldots, p_{t}\right)$. Then up to isomorphism the indecomposable $\Lambda$-modules of rank 1 are uniquely determined by their dimension vectors and are classified by all the sequences of the form $\left(u ;\left(u_{i}\right)\right):=$ $\left(u ; u_{1}, \ldots, u_{t}\right) \in \mathbb{N} \times\left(\left[p_{1}\right] \times \cdots \times\left[p_{t}\right]\right)$. In particular observe that for any such sequence we have the indecomposable matrix $\Lambda$-module $M^{\left(u ;\left(u_{i}\right)\right)}=\left(M_{\alpha_{j}^{(i)}}\right.$, where $M_{\alpha_{u_{1}}^{(1)}}=X_{u} \in \mathbb{M}_{(u+1) \times u}(k), M_{\alpha_{u_{2}}^{(2)}}=Y_{u} \in \mathbb{M}_{(u+1) \times u}(k)$ and $M_{\alpha_{u_{i}}^{(i)}}=X_{u}+\lambda_{i} Y_{u}$, for $i=$ $3, \ldots, t$, whereas $M_{\alpha_{j}^{(i)}}$ for all the remaining other pairs $(i, j)$ are equal to the identity matrices, $I_{u}$ or $I_{u+1}$, respectively (cf. [17, Proposition 4.3]; our construction is slightly different). In the formulas above, by $X_{u}$ and $Y_{u}$ we mean as usually the matrices $X_{u}:=\left[\begin{array}{ccc}1 & & \\ & \ddots & \\ & & 1 \\ 0 & \cdots & 0\end{array}\right], \quad Y_{u}:=\left[\begin{array}{ccc}0 & \cdots & \\ 1 & & \\ & \ddots & \\ & & 1\end{array}\right] \in \mathbb{M}_{(u+1) \times u}(k)$.

Let now $\Lambda$ be a tubular canonical algebra. Consider the family of subsets $\Theta^{(d)}=$ $\Theta_{\mathbf{p}}^{(d)} \subset \mathbb{Z} \times\left(\left[p_{1}\right] \times \cdots \times\left[p_{t}\right]\right), d \in \mathbb{Z}_{\geq p}$, defined as follows:

- $\Theta^{(p)}, \ldots, \Theta^{(2 p-1)}$ are as in the table below

\begin{tabular}{c|l}
$\mathbf{p}$ & $\Theta^{(i)}$, for $i=p, \ldots, 2 p-1$ \\
\hline$(2,2,2,2)$ & $\Theta^{(2)}:(0 ; 1,1,2,2),(0 ; 2,2,1,1) ; \quad \Theta^{(3)}:(1 ; 1,1,2,1),(0 ; 2,2,1,2)$ \\
\hline$(3,3,3)$ & $\Theta^{(3)}:(0 ; 1,2,3),(0 ; 2,3,1),(0 ; 3,1,2)$ \\
& $\Theta^{(4)}:(1 ; 1,2,1),(0 ; 2,3,2),(0 ; 3,1,3)$ \\
& $\Theta^{(5)}:(1 ; 1,2,2),(0 ; 2,3,3),(1 ; 3,1,1)$ \\
\hline$(2,4,4)$ & $\Theta^{(4)}:(0 ; 1,2,4),(0 ; 2,3,1),(0 ; 1,4,2),(0 ; 2,1,3)$ \\
& $\Theta^{(5)}:(1 ; 1,2,1),(0 ; 2,3,2),(0 ; 1,4,3),(0 ; 2,1,4)$ \\
& $\Theta^{(6)}:(1 ; 1,2,2),(0 ; 2,3,3),(0 ; 1,4,4),(1 ; 2,1,1)$ \\
& $\Theta^{(7)}:(1 ; 1,2,3),(0 ; 2,3,4),(1 ; 1,4,1),(1 ; 2,1,2)$ \\
\hline$(2,3,6)$ & $\Theta^{(6)}:(1 ; 1,1,1),(0 ; 2,2,2),(0 ; 1,3,3),(0 ; 2,1,4),(0 ; 1,2,5),(-1 ; 2,3,6)$ \\
& $\Theta^{(7)}:(1 ; 1,1,2),(0 ; 2,2,3),(0 ; 1,3,4),(0 ; 2,1,5),(0 ; 1,2,6),(0 ; 2,3,1)$ \\
& $\Theta^{(8)}:(1 ; 1,1,3),(0 ; 2,2,4),(0 ; 1,3,5),(0 ; 2,1,6),(1 ; 1,2,1),(0 ; 2,3,2)$ \\
& $\Theta^{(9)}:(1 ; 1,1,4),(0 ; 2,2,5),(0 ; 1,3,6),(1 ; 2,1,1),(1 ; 1,2,2),(0 ; 2,3,3)$ \\
& $\Theta^{(10)}:(1 ; 1,1,5),(0 ; 2,2,6),(1 ; 1,3,1),(1 ; 2,1,2),(1 ; 1,2,3),(0 ; 2,3,4)$ \\
& $\Theta^{(11)}:(1 ; 1,1,6),(1 ; 2,2,1),(1 ; 1,3,2),(1 ; 2,1,3),(1 ; 1,2,4),(0 ; 2,3,5)$
\end{tabular}

- $\quad \Theta^{(d)}$, for $d \geq 2 p$, are determined by the condition:

$$
\left(u ; u_{1}, \ldots, u_{t}\right) \in \Theta^{(d)} \Leftrightarrow\left(u-(c-1) ; u_{1}, \ldots, u_{t}\right) \in \Theta^{(p+e)}
$$

where $c=$ quo $_{p} d$ and $e=\operatorname{rem}_{p} d$.

(Note that $\Theta_{\mathbf{p}}^{(d)} \subset \mathbb{N} \times\left(\left[p_{1}\right] \times \cdots \times\left[p_{t}\right]\right)$, except the case $d=p$, for $\mathbf{p}=(2,3,6)$ ).

For any $d \in \mathbb{Z}_{\geq p}$ (resp. $d \in \mathbb{Z}_{\geq 7}$, if $\left.\mathbf{p}=(2,3,6)\right)$, we denote by $\mathcal{F}^{(d)}$ the full subcategory formed by the rank-1 $\Lambda$-modules $M^{\left(u ;\left(u_{i}\right)\right)}$, for all $\left(u ;\left(u_{i}\right)\right) \in \Theta^{(d)}$. 
Lemma For any pair $(\mathbf{p}, d)$ as above $\mathcal{F}^{(d)}$ consists of p objects and is a semisimple category of the form $\mathcal{F}(\mathcal{T})$ consisting of all quasi-simple objects in some nonhomogeneous stable tube $\mathcal{T}$ of maximal rank $\rho_{\mathcal{T}}=p$ in $\mathcal{X}_{d}$.

We give a short proof of this fact in Section 5.2.

Let $B$ be a $k[\xi] \otimes_{k} \Lambda$-module from $\bmod _{k[\xi]} \Lambda$. Then for any $d$ as above we denote by $\Upsilon_{d}(B)$ the middle term $\Upsilon_{\widetilde{\mathcal{F}}^{(d)}}(B)$ of the universal extension $\eta_{B}^{\widetilde{\mathcal{F}}^{(d)}}$ of $B$ with respect to the $k[\xi]$-semisimple category $\widetilde{\mathcal{F}}^{(d)}:=\{k[\xi] \otimes F\}_{F \in \mathcal{F}^{(d)}}$ in $\bmod _{k[\xi]} \Lambda$, if it exists. We will use the abbreviated notation $\left(\Upsilon_{d} \circ \Upsilon_{d^{\prime}}\right)(B)$ for $\Upsilon_{d}\left(\Upsilon_{d^{\prime}}(B)\right)$, if $\Upsilon_{d^{\prime}}(B)$ and $\Upsilon_{d}\left(\Upsilon_{d^{\prime}}(B)\right)$ exist; similarly, $\Upsilon_{d}^{c}(B)$ for $\left(\Upsilon_{d} \circ \cdots \circ \Upsilon_{d}\right)(B)$, where $c \in \mathbb{N}_{+}$.

Let $q \in \mathbb{Q}_{>0}$ be a positive rational number and $q=\frac{a}{b}$, with $a, b \in \mathbb{Z}_{\geq 1}$, the irreducible fraction presentation of $q$. With $q$ we associate the number $n=n(q) \in$ $\mathbb{N}$ and two sequences $a(q):=\left(a_{0}, a_{1}, \ldots, a_{n+1}, a_{n+2}\right)$ and $c(q):=\left(c_{1}, \ldots, c_{n+1}\right)$ of nonnegative integers. They are inductively defined by the formulas $c_{i}=$ quo $_{a_{i}} a_{i-1}$ and $a_{i+1}=\operatorname{rem}_{a_{i}} a_{i-1}$, for $i=1, \ldots, n+1$, where $a_{0}=a, a_{1}=b, a_{n+1}=1, a_{n+2}=0$, and are formed when Euclidean algorithm for computing $\operatorname{gcd}(a, b)$ is executed.

For $d \in \mathbb{Z}_{\geq p}$, by $B^{(d)}$ we always mean the matrix bimodule in $\bmod _{k[\xi]}^{\text {mat }} \Lambda$ constructed in [9] such that $\left(B^{(d)}\right)_{f}$, for $f=f_{\mathbf{p}}$, is a parametrizing bimodule for $\mathcal{X}_{d}^{0}$ (see [9, Theorem 4.1 and Table 4.7]).

Now we can formulate the main result of this paper.

Main Theorem Let $\Lambda$ be a tubular canonical algebra of the type $\mathbf{p}=\left(p_{1}, \ldots, p_{t}\right)$ and $f=f_{\mathbf{p}}$.

(a) For any $q \in \mathbb{Q}_{\geq p}$, if $\mathbf{p}=(2,2,2,2),(3,3,3)$, $(2,4,4)$; respectively, $q \in\{6\} \cup$ $\mathbb{Q}_{\geq 7}$, if $\mathbf{p}=(2,3,6)$, the $k[\xi] \otimes_{k} \Lambda$-module $B^{(q)}$ given by the formula

$$
B^{(q)}:= \begin{cases}\left(\Upsilon_{c_{1}}^{c_{2}} \cdots \cdots \circ \Upsilon_{c_{1}+c_{3} \cdots+c_{2 m-1}}^{c_{2 m}}\right)\left(B^{\left(c_{1}+c_{3}+\cdots+c_{2 m+1}\right)}\right) & \text { if } n=2 m, \\ \left.\left(\Upsilon_{c_{1}}^{c_{2}} \circ \cdots \circ \Upsilon_{c_{1}+c_{3} \cdots+c_{2 m-1}}^{c_{2 m}}\right) \circ \Upsilon_{c_{1}+c_{3} \cdots+c_{2 m+1}}^{c_{2 m+2}-1}\right) & \text { if } n=2 m+1 . \\ \quad \times\left(B^{\left(c_{1}+c_{3}+\cdots+c_{2 m+1}+1\right)}\right) & \end{cases}
$$

where $c(q)=\left(c_{1}, \ldots c_{n+1}\right)$, is well defined, belongs to $\bmod _{k[\xi]} \Lambda$ and its localization $\left(B^{(q)}\right)$ f yields a parametrizing bimodule for all indecomposable $\Lambda$-modules from the family of all homogeneous tubes in $\mathcal{X}_{q}$.

(b) If $\mathbf{p}=(2,3,6)$ then for any $6<q=\frac{a}{b}<7$ with $a, b \in \mathbb{Z}_{\geq 1}$, the fraction $\hat{q}:=$ $\frac{64 b-9 a}{7 b-a}$ belongs to $\mathbb{Q}_{\geq 7}$, the $k[\xi] \otimes_{k} \Lambda$-module

$$
B^{(q)}:=\operatorname{Ker}(\text { can })
$$

where $\quad \operatorname{can}=\operatorname{can}_{B^{(\hat{q})}}^{\widetilde{\mathcal{F}}^{(8)}}: \bigoplus_{\widetilde{F}_{\mathrm{ob}} \tilde{\mathcal{F}}^{(8)}} \operatorname{Hom}_{k[\xi] \otimes \Lambda}\left(\widetilde{F}, B^{(\hat{q})}\right) \otimes_{k[\xi]} \widetilde{F} \rightarrow B^{(\hat{q})}$ is the canonical map and $B^{(\hat{q})}$ is a $k[\xi] \otimes_{k} \Lambda$-module in $\bmod _{k[\xi]} \Lambda$ defined for $\hat{q}$ as in (a), belongs to $\bmod _{k[\xi]} \Lambda$ and its localization $\left(B^{(q)}\right)_{f}$ yields a parametrizing bimodule for all indecomposable $\Lambda$-modules from the family of all homogeneous tubes in $\mathcal{X}_{q}$. 
Note that if $q \in \mathbb{Z}_{\geq p}$ then $a_{2}=0, n=0$, so the formula says that $B^{(q)}=B^{\left(c_{1}\right)}$ (clearly, $q=c_{1}$ ). Observe also that the integers $c_{1}+c_{3}+\cdots+c_{2 m+1}$ and $c_{1}+c_{3}+$ $\cdots+c_{2 m+1}+1$ belong to $\mathbb{Z}_{\geq p}$ (resp. in $\mathbb{Z}_{\geq 7}$, if $q \geq 7$ ); moreover, all the integers of the form $c_{1}+c_{3}+\cdots+c_{2 j+1}$, for $j \geq 0$, belong to $\mathbb{Z}_{\geq p}$ (resp. in $\mathbb{Z}_{\geq 7}$, if $q \geq 7$ ).

To prove this result we use the previous theorems and also some information on the "slope combinatorics" as well as nice properties of the kernel construction, which we discuss in Section 5. The proof of Theorem 3.3 will be given in Sections 5.3 and 5.6.

Corollary For any $q \in \mathbb{Q}_{\leq 0}$, the localization $\left(B^{(q)}\right)_{f}$ of the $k[\xi] \otimes \Lambda^{\mathrm{op}}$-module $B^{(q)}:=\operatorname{Hom}_{k[\xi]}\left(B^{(p-q)}, k[\xi]\right)$, regarded as a module in $\bmod _{R} \Lambda$ via the canonical k-algebra isomorphism $\Lambda \stackrel{\sim}{\rightarrow} \Lambda^{\mathrm{op}}$ (see [9, 4.1] for details), yields a parametrizing bimodule for $\mathcal{X}_{q}^{0}$.

Proof Follows immediately from the theorem by the arguments as in [9, 4.2].

\section{Universal Extensions of Bimodules}

In this section we introduce the notion of a universal extension in the categories $\mathcal{C}$ of modules over $R$-algebras $\Xi$, where $R$ is a commutative $k$-algebra, and we discuss its properties necessary for the proof of Theorem 3.1, in case $\Xi=R \otimes_{k} \Lambda$, where $\Lambda$ is a finite dimensional $k$-algebra.

\section{1}

We start with a rather general situation. Let $R$ be a commutative ring, $\mathcal{C}$ an abelian $R$-category, admitting the functor $\otimes_{R}:(\bmod R) \times \mathcal{C} \rightarrow \mathcal{C}($ e.g. $\mathcal{C}=$ MOD $\Xi$, where $\Xi$ is an $R$-algebra), and $\mathcal{F}$ a full subcategory of $\mathcal{C}$ with nonisomorphic distinct objects. Denote by $\mathcal{F}^{!}$the additive closure of $\mathcal{F}$ in $\mathcal{C}$ in the sense of finite direct sums and isomorphisms. Then for an object $B$ in $\mathcal{C}$ we can ask, if the functor

$$
\operatorname{Ext}_{\mathcal{C}}^{1}(B,-)_{\mid \mathcal{F}^{!}}: \mathcal{F}^{!} \rightarrow \operatorname{MOD} R
$$

is representable; equivalently (by the Yoneda lemma), if there exist an object $\Omega_{\mathcal{F}}(B)$ in $\mathcal{F}^{!}$and an exact sequence

$$
\eta_{B}^{\mathcal{F}}: \quad 0 \rightarrow \Omega_{\mathcal{F}}(B) \longrightarrow \Upsilon_{\mathcal{F}}(B) \longrightarrow B \rightarrow 0
$$

in $\mathcal{C}$ with the property, that for any object $F \in \mathcal{F}^{!}$and any extension $[\eta] \in \operatorname{Ext}_{\mathcal{C}}^{1}(B, F)$ there exists a unique morphism $g \in \operatorname{Hom}_{\mathcal{C}}\left(\Omega_{\mathcal{F}}(B), F\right)$ such that $[\eta]=g \cdot\left[\eta_{B}^{\mathcal{F}}\right]$.

The extension $\eta_{B}^{\mathcal{F}}$ with the property as above (if it exists) is called a co-universal extension of $B$ with respect to $\mathcal{F}$. In this paper we will use simply the name universal extension.

The problem of the existence of universal extensions has a rather simple solution for certain natural classes of subcategories $\mathcal{F}$.

Recall that a subcategory $\mathcal{F}=\left\{F_{i}\right\}_{i \in I}$ of $\mathcal{C}$ is called $R$-semisimple, if

$$
\operatorname{Hom}_{\mathcal{C}}\left(F_{i}, F_{j}\right) \cong \begin{cases}R & \text { if } i=j, \\ 0 & \text { if } i \neq j\end{cases}
$$


(Note that then each $F_{i}$ is indecomposable, if $R$ is a domain or local artinian; moreover, that $\mathcal{F}$ is semisimple if $R$ is a field).

We denote by $D^{R}$ the functor $\operatorname{Hom}_{R}(-, R):$ MOD $R \rightarrow$ MOD $R$. If $E$ is a finitely generated free $R$-module and $\mathcal{E}=\left\{e_{1}, \ldots, e_{m}\right\}$ is an $R$-base of $E$ then by $\mathcal{E}^{R}$ we denote the dual base for $\mathcal{E}$, i.e. the set $\mathcal{E}^{R}:=\left\{e_{j}^{R}: E \rightarrow R\right\}_{j=1, \ldots, m}$ consisting of the canonical projections with respect to the base $\mathcal{E}$. In case $R$ is a field, we use the classical notation, $D$ for $D^{R}, \mathcal{E}^{*}$ and $e_{j}^{*}$ for $\mathcal{E}^{R}$ and $e_{j}^{R}$, respectively.

Theorem Let $R$ be a commutative ring and $\mathcal{F}=\left\{F_{1}, \ldots, F_{\rho}\right\}$ an $R$-semisimple finite subcategory of $\mathcal{C}$. The universal extension $\eta_{B}^{\mathcal{F}}$, for an object $B$ in $\mathcal{C}$, exists if and only if $\operatorname{Ext}_{\mathcal{C}}^{1}\left(B, F_{i}\right) \cong R^{m_{i}}$ for every $i=1, \ldots, \rho$, where $m_{i} \in \mathbb{N}$. In this case we can assume that

$$
\Omega_{\mathcal{F}}(B):=\bigoplus_{i=1}^{\rho} D^{R}\left(\operatorname{Ext}_{\mathcal{C}}^{1}\left(B, F_{i}\right)\right) \otimes_{R} F_{i}
$$

and then for any collection $\mathcal{E}:=\left\{\mathcal{E}_{i}\right\}_{i=1, \ldots, \rho}$ of bases of the $R$-modules $\operatorname{Ext}_{\mathcal{C}}^{1}\left(B, F_{i}\right)$

$$
\left[\eta_{B}^{\mathcal{F}}\right]=\varepsilon \cdot e
$$

where $e:=e(\mathcal{E}) \in \operatorname{Ext}_{\mathcal{C}}^{1}\left(B, \bigoplus_{i=1}^{\rho} \bigoplus_{j=1}^{m_{i}} F_{i}\right) \quad\left(=\bigoplus_{i=1}^{\rho} \bigoplus_{j=1}^{m_{i}} \operatorname{Ext}_{\mathcal{C}}^{1}\left(B, F_{i}\right)\right)$ is the extension whose components $e_{j}^{(i)} \in \operatorname{Ext}_{\mathcal{C}}^{1}\left(B, F_{i}\right)$ are given by $\mathcal{E}_{i}$ in the sense that $\mathcal{E}_{i}=$ $\left\{e_{j}^{(i)}\right\}_{j=, 1, \ldots, m_{i}}$ for every $i=1, \ldots, \rho$, and

$$
\varepsilon=\varepsilon(\mathcal{E}): \bigoplus_{i=1}^{\rho} \bigoplus_{j=1}^{m_{i}} F_{i} \rightarrow \Omega_{\mathcal{F}}(B)
$$

is the diagonal $R$-isomorphism $\varepsilon:=\bigoplus_{i=1}^{\rho} \varepsilon_{i}$ whose components are row matrix maps $\varepsilon_{i}:=\left[\left(e_{j}^{(i)}\right)^{R} \otimes-\right]_{j}: \bigoplus_{j=1}^{m_{i}} F_{i} \rightarrow D^{R}\left(\operatorname{Ext}_{\mathcal{C}}^{1}\left(B, F_{i}\right)\right) \otimes_{R} F_{i}$, for $i=1, \ldots, \rho$, defined by the dual bases $\mathcal{E}_{i}^{R}:=\left\{\left(e_{j}^{(i)}\right)^{R}\right\}_{j=1, \ldots, m_{i}}$ for $\mathcal{E}_{i}$.

Proof We give only a brief sketch of the proof which is in principle similar as in the case $R=k$ (cf. [14]). The necessity of the "Ext-condition" follows immediately, since $\Omega_{\mathcal{F}}(B) \cong \bigoplus_{i=1}^{\rho} F_{i}^{m_{i}^{\prime}}$, for some $m_{i}^{\prime} \in \mathbb{N}$, so by our assumptions we have $\operatorname{Ext}_{\mathcal{C}}^{1}\left(B, F_{i}\right) \cong$ $\operatorname{Hom}_{\mathcal{C}}\left(\Omega_{\mathcal{F}}(B), F_{i}\right) \cong R^{m_{i}^{\prime}}$.

To prove the converse implication, for a fixed $B$ we consider the composed (natural with respect to $B$ ) $R$-homomorphisms $\zeta_{i}^{\prime}$

$$
E_{i} \rightarrow E_{i} \otimes_{R} \operatorname{Hom}_{\mathcal{C}}\left(F_{i}, F_{i}\right) \rightarrow \operatorname{Hom}_{\mathcal{C}}\left(D^{R}\left(E_{i}\right) \otimes_{R} F_{i}, F_{i}\right)
$$

given by the formula $\zeta_{i}^{\prime}(e)(\gamma \otimes f)=\gamma(e) f$, for $e \in E_{i}, f \in F_{i}$ and $\gamma \in D^{R}\left(E_{i}\right)$, where $E_{i}:=\operatorname{Ext}_{\mathcal{C}}^{1}\left(B, F_{i}\right)$, for $i=1, \ldots, \rho$. Note that by our assumptions $\zeta_{i}^{\prime}$ is an isomorphism. Denote by $\zeta_{F_{i}}$ the map

$$
E_{i} \stackrel{\zeta_{i}^{\prime}}{\longrightarrow} \operatorname{Hom}_{\mathcal{C}}\left(D^{R}\left(E_{i}\right) \otimes_{R} F_{i}, F_{i}\right) \stackrel{w_{i}}{\longrightarrow} \operatorname{Hom}_{\mathcal{C}}\left(\Omega_{\mathcal{F}}(B), F_{i}\right)
$$

where $\Omega_{\mathcal{F}}(B):=\bigoplus_{i=1}^{\rho} D^{R}\left(E_{i}\right) \otimes_{R} F_{i}$ and $w_{i}$ is induced by the standard projection $\pi_{i}: \Omega_{\mathcal{F}}(B) \rightarrow D^{R}\left(E_{i}\right) \otimes_{R} F_{i}$. 
The family $\left\{\zeta_{F_{i}}\right\}_{i=1, \ldots, \rho}$ yields an isomorphism of functors

$$
\operatorname{Ext}_{\mathcal{C}}^{1}(B,-)_{\mid \mathcal{F}} \cong \operatorname{Hom}_{\mathcal{C}}\left(\Omega_{\mathcal{F}}(B),-\right)_{\mid \mathcal{F}}
$$

( $\mathcal{F}$ is $R$-semisimple!), and in consequence it extends (not uniquely) to an isomorphism $\zeta:=\left\{\zeta_{F}\right\}_{F \in \text { ob } \mathcal{F} \text { : of functors }}$

$$
\operatorname{Ext}_{\mathcal{C}}^{1}(B,-)_{\mid \mathcal{F} !} \cong \operatorname{Hom}_{\mathcal{C}}\left(\Omega_{\mathcal{F}}(B),-\right)_{\mid \mathcal{F} !}
$$

To show that

$$
\left[\eta_{B}^{\mathcal{F}}\right]:=\zeta_{\Omega_{\mathcal{F}}(B)}^{-1}\left(\operatorname{id}_{\Omega_{\mathcal{F}}(B)}\right)
$$

has the form as in the statement observe that by definition of $\zeta$, for any $e^{\prime} \in E_{i}$ we have the equality

$$
e^{\prime}=\left[\zeta_{F_{i}}\left(e^{\prime}\right) \cdot \eta_{B}^{\mathcal{F}}\right]
$$

Now fix a collection $\mathcal{E}$ consisting of bases $\mathcal{E}_{i}=\left\{e_{j}^{(i)}\right\}_{j=, 1, \ldots, m_{i}}$ of the $R$-modules $\operatorname{Ext}_{\mathcal{C}}^{1}\left(B, F_{i}\right)$, for $i=1, \ldots, \rho$. Then for any $i$ we have the equality

$$
\sum_{j=1}^{m_{i}}\left(\left(e_{j}^{(i)}\right)^{R} \otimes-\right) \circ\left(\zeta_{i}^{\prime}\left(e_{j}^{(i)}\right)\right)=\mathrm{id}_{D^{R}\left(E_{i}\right) \otimes_{R} F_{i}}
$$

Then applying the formulas (ii) and (iii) we get

$$
\begin{aligned}
{\left[\eta_{B}^{\mathcal{F}}\right] } & =\left[\operatorname{id}_{\Omega_{\mathcal{F}}(B)} \cdot \eta_{B}^{\mathcal{F}}\right]=\sum_{i=1}^{\rho}\left(\iota_{i} \operatorname{id}_{D^{R}\left(E_{i}\right) \otimes F_{i}} \pi_{i}\right) \cdot\left[\eta_{B}^{\mathcal{F}}\right] \\
& =\sum_{i=1}^{\rho} \iota_{i}\left(\sum_{j=1}^{m_{i}}\left(\left(e_{j}^{(i)}\right)^{R} \otimes-\right) \cdot e_{j}^{(i)}\right)=\sum_{i=1}^{\rho} \iota_{i}\left(\varepsilon_{i}\left(\left(e_{j}^{(i)}\right)_{j}\right)\right)=\varepsilon \cdot e
\end{aligned}
$$

where $\iota_{i}: D^{R}\left(E_{i}\right) \otimes_{R} F_{i} \rightarrow \Omega_{\mathcal{F}}(B)$ denotes the canonical embedding, for $i=$ $1, \ldots, \rho$, and $e \in \operatorname{Ext}_{\mathcal{C}}^{1}\left(B, \bigoplus_{i=1}^{\rho} \bigoplus_{j=1}^{m_{i}} F_{i}\right)$ is the extension with the components $e_{j}^{(i)} \in$ $\operatorname{Ext}_{\mathcal{C}}^{1}\left(B, F_{i}\right)$ and $\varepsilon=\varepsilon(\mathcal{E})\left(=\bigoplus_{i=1}^{\rho} \varepsilon_{i}\right)$ is as in the statement of the theorem.

Finally, note that the value of $\left[\eta_{B}^{\mathcal{F}}\right]$ does not depend on the choice of the isomorphism $\zeta$ (cf. formulas $(* *)$ and (i)).

Remark If we know that $\eta_{B}^{\mathcal{F}}$ exists then the formulas $(*)$ and (**), for a fixed base collection $\mathcal{E}=\left\{\mathcal{E}_{i}\right\}_{i=1, \ldots, \rho}$, can be used as a definition of $\left[\eta_{B}^{\mathcal{F}}\right]$.

From now on we will work with the category $\mathcal{C}=\bmod R \otimes_{k} \Lambda$, where $\Lambda=k Q / I$ is the $k$-algebra of a bound quiver $(Q, I)$, and $R$ is a commutative $k$-algebra. Without loss of generality we can assume that $I$ is generated by a finite set $R e l$, consisting of relations $\varrho_{i}=\sum_{j=1}^{t_{i}} \lambda_{i, j} \alpha_{p_{i, j}}^{(i, j)} \ldots \alpha_{1}^{(i, j)}$, with starting and terminus vertices $s\left(\varrho_{i}\right)$ and $t\left(\varrho_{i}\right)$, respectively, for $i=1, \ldots, \mid$ Rel $\mid$.

A crucial role in our considerations plays the classical description of the extension groups $\operatorname{Ext}_{R \otimes \Lambda}^{1}\left(B, B^{\prime}\right)$, for $R \otimes_{k} \Lambda$-modules $B, B^{\prime}$, in terms of Hochschild cohomologies; more precisely, derivation modules. 
Let $B, B^{\prime}$ be a pair of $R \otimes_{k} \Lambda$-modules from $\bmod R \otimes_{k} \Lambda$. We consider the $R$-modules of derivations and inner derivations of $\Lambda$ in the $\Lambda-\Lambda$ bimodule $\operatorname{Hom}_{R}\left(B, B^{\prime}\right):=\bigoplus_{x, y \in Q_{0}} \operatorname{Hom}_{R}\left(B_{x}, B_{y}^{\prime}\right)$, respectively. The module $\operatorname{Der}\left(\Lambda, \operatorname{Hom}_{R}\left(B, B^{\prime}\right)\right)$ consists of all sequences $\delta=\left(\delta_{\alpha}\right) \in \bigoplus_{\alpha \in Q_{1}} \operatorname{Hom}_{R}\left(B_{t(\alpha)}\right.$, $\left.B_{s(\alpha)}^{\prime}\right)$, satisfying the equalities

$$
\sum_{j=1}^{t_{i}} \sum_{u=1}^{p_{i, j}} \lambda_{i, j} B_{\alpha_{1}^{(i, j)}}^{\prime} \ldots B_{\alpha_{u-1}^{(i, j)}}^{\prime} \delta_{\alpha_{u}} B_{\alpha_{u+1}^{(i, j)}} \ldots B_{\alpha_{p_{i, j}}^{(i, j)}}=0
$$

for all $i=1, \ldots,|\operatorname{Rel}|$, and $\operatorname{Der}^{0}\left(\Lambda, \operatorname{Hom}_{R}\left(B, B^{\prime}\right)\right)$ of all $\delta=\left(\delta_{\alpha}\right)$ with the property that there exists a sequence $\chi=\left(\chi_{x}\right) \in \bigoplus_{x \in Q_{0}} \operatorname{Hom}_{R}\left(B_{x}, B_{x}^{\prime}\right)$ such that

$$
\delta_{\alpha}=\chi_{s(\alpha)} B_{\alpha}-B_{\alpha}^{\prime} \chi_{t(\alpha)}
$$

in $\operatorname{Hom}_{R}\left(B_{t(\alpha)}, B_{s(\alpha)}^{\prime}\right)$, for every $\alpha \in Q_{1}$.

To any $\delta \in \operatorname{Der}\left(\Lambda, \operatorname{Hom}_{R}\left(B, B^{\prime}\right)\right)$ we associate the extension

$$
\eta(\delta): \quad 0 \rightarrow B^{\prime} \rightarrow E \rightarrow B \rightarrow 0
$$

in $\bmod R \otimes_{k} \Lambda$, with $E=E(\delta)$, given by $E_{x}=B_{x}^{\prime} \oplus B_{x}$ and $E_{\alpha}=\left[\begin{array}{cc}B_{\alpha}^{\prime} & \delta_{\alpha} \\ 0 & B_{\alpha}\end{array}\right]$, for $x \in$ $Q_{0}$ and $\alpha \in Q_{1}$.

Lemma For $B$ and $B^{\prime}$ as above, the mapping $\delta \mapsto \eta(\delta)$ induces an R-monomorphism

$$
\operatorname{Der}\left(\Lambda, \operatorname{Hom}_{R}\left(B, B^{\prime}\right)\right) / \operatorname{Der}^{0}\left(\Lambda, \operatorname{Hom}_{R}\left(B, B^{\prime}\right)\right) \rightarrow \operatorname{Ext}_{R \otimes \Lambda}^{1}\left(B, B^{\prime}\right)
$$

which is an isomorphism, provided B belongs to $\bmod _{R} \Lambda$.

Proof Repeat the arguments from the classical situation $R=k$ (see [3]).

Now we discuss the behaviour of universal extensions of $R \otimes_{k} \Lambda$-modules with respect to extension and restriction of the coefficient ring. For this aim we fix the following notation.

Let $h: R \rightarrow \bar{R}$ be a homomorphism of commutative $k$-algebras. Then the functor $\bar{R} \otimes_{R}-:$ MOD $R \rightarrow$ MOD $\bar{R}$ induces functors MOD $R \otimes_{k} \Lambda \rightarrow \operatorname{MOD} \bar{R} \otimes_{k} \Lambda$ and $\bmod _{R} \Lambda \rightarrow \bmod _{\bar{R}} \Lambda$, which we denote also by $\bar{R} \otimes_{R}-$. Consequently, for $B, B^{\prime}$ in $\bmod R \otimes_{k} \Lambda$, we have an induced $R$-homomorphism

$$
h_{B, B^{\prime}}^{\text {hom }}: \operatorname{Hom}_{R \otimes \Lambda}\left(B, B^{\prime}\right) \rightarrow \operatorname{Hom}_{\bar{R} \otimes \Lambda}\left(\bar{R} \otimes_{R} B, \bar{R} \otimes_{R} B^{\prime}\right)
$$

and in case $B$ is in $\bmod _{R} \Lambda$, also

$$
h_{B, B^{\prime}}^{\text {ext }}: \operatorname{Ext}_{R \otimes \Lambda}^{1}\left(B, B^{\prime}\right) \rightarrow \operatorname{Ext}_{\bar{R} \otimes \Lambda}^{1}\left(\bar{R} \otimes_{R} B, \bar{R} \otimes_{R} B^{\prime}\right)
$$

mapping an exact sequence

$$
e: \quad 0 \rightarrow B^{\prime} \stackrel{g_{1}}{\longrightarrow} E \stackrel{g_{2}}{\longrightarrow} B \rightarrow 0
$$

in $\bmod R \otimes_{k} \Lambda$ to the sequence

$$
\bar{R} \otimes_{R} e: \quad 0 \rightarrow \bar{R} \otimes_{R} B^{\prime} \stackrel{\bar{g}_{1}}{\longrightarrow} \bar{R} \otimes_{R} E \stackrel{\bar{g}_{2}}{\longrightarrow} \bar{R} \otimes_{R} B \rightarrow 0
$$


in $\bmod \bar{R} \otimes_{k} \Lambda$, where $\bar{g}_{1}=\bar{R} \otimes_{R} g_{1}$ and $\bar{g}_{2}=\bar{R} \otimes_{R} g_{2}$, which is exact, since $e$ splits in $\bmod R$. It is easy to observe that they induce in a standard way $\bar{R}$-homomorphisms

$$
\breve{h}_{B, B^{\prime}}^{\text {hom }}: \bar{R} \otimes_{R} \operatorname{Hom}_{R \otimes \Lambda}\left(B, B^{\prime}\right) \rightarrow \operatorname{Hom}_{\bar{R} \otimes \Lambda}\left(\bar{R} \otimes_{R} B, \bar{R} \otimes_{R} B^{\prime}\right)
$$

and

$$
\breve{h}_{B, B^{\prime}}^{\text {ext }}: \bar{R} \otimes_{R} \operatorname{Ext}_{R \otimes \Lambda}^{1}\left(B, B^{\prime}\right) \rightarrow \operatorname{Ext}_{\bar{R} \otimes \Lambda}^{1}\left(\bar{R} \otimes_{R} B, \bar{R} \otimes_{R} B^{\prime}\right)
$$

respectively.

The question when $\breve{h}_{B, B^{\prime}}$ and $\breve{h}^{\text {ext }}{ }_{B, B^{\prime}}$ are $\bar{R}$-isomorphisms is very important in the context of the existence of universal extensions.

Lemma If $R$ is a field $k$ then the $\bar{R}$-homomorphism $\breve{h}_{B, B^{\prime}}^{\text {hom }}$ is an isomorphism for any pair $B, B^{\prime}$ in $\bmod R \otimes_{k} \Lambda(=\bmod \Lambda)$.

Proof For any pair $V, V^{\prime}$ of $k$-spaces such that $\operatorname{dim}_{k} V$ is finite consider the following composed $\bar{R}$-isomorphism $\vartheta=\vartheta_{V, V^{\prime}}$ :

$$
\begin{gathered}
\bar{R} \otimes_{k} \operatorname{Hom}_{k}\left(V, V^{\prime}\right) \cong \operatorname{Hom}_{k}\left(V, \bar{R} \otimes_{k} V^{\prime}\right) \cong \\
\operatorname{Hom}_{k}\left(V, \operatorname{Hom}_{\bar{R}}\left(\bar{R}, \bar{R} \otimes_{k} V^{\prime}\right)\right) \cong \operatorname{Hom}_{\bar{R}}\left(\bar{R} \otimes_{k} V, \bar{R} \otimes_{k} V^{\prime}\right)
\end{gathered}
$$

where the first homomorphism is given by the mapping $s \otimes g \mapsto s \otimes g(-)$, for $s \in \bar{R}$ and $g \in \operatorname{Hom}_{k}\left(V, V^{\prime}\right)$ (it is an isomorphism, since $\operatorname{dim}_{k} V$ is finite!), the second one is induced by the standard $\bar{R}$-isomorphism $\operatorname{Hom}_{\bar{R}}(\bar{R}, M) \cong M$, for the $\bar{R}$-module $M=$ $\bar{R} \otimes_{k} V^{\prime}$, and the third one is the respective adjunction formula. It is easy to check that

$$
\vartheta\left(s_{1} \otimes g\right)\left(s_{2} \otimes v\right)=s_{1}\left(s_{2} \otimes g(v)\right)
$$

for any $s_{1}, s_{2} \in \bar{R}, g \in \operatorname{Hom}_{k}\left(V, V^{\prime}\right)$ and $v \in V$.

Observe now that if $B, B^{\prime}$ is a pair of $\Lambda$-modules such that $B$ is finite dimensional, then $\vartheta_{B, B^{\prime}}$ induces an $\bar{R}$-isomorphism

$$
\left(\vartheta_{B, B^{\prime}}\right)_{\mid}: \bar{R} \otimes_{k} \operatorname{Hom}_{R \otimes \Lambda}\left(B, B^{\prime}\right) \rightarrow \operatorname{Hom}_{\bar{R} \otimes \Lambda}\left(\bar{R} \otimes_{R} B, \bar{R} \otimes_{R} B^{\prime}\right)
$$

(the isomorphisms $\vartheta_{V, V^{\prime}}$ are natural!). In consequence, $\breve{h}_{B, B^{\prime}}^{\text {hom }}$ is an $\bar{R}$-isomorphism, since $\breve{h}_{B, B^{\prime}}^{\text {hom }}=\left(\vartheta_{B, B^{\prime}}\right)$, by the formula (i).

Remark Similarly, we have also $R$-homomorphisms $h_{B, B^{\prime}}^{d e r} \operatorname{Der}\left(\Lambda, \operatorname{Hom}_{R}\right.$ $\left.\left(B, B^{\prime}\right)\right) \rightarrow \operatorname{Der}\left(\Lambda, \operatorname{Hom}_{\bar{R}}\left(\bar{R} \otimes_{R} B, \bar{R} \otimes_{R} B^{\prime}\right)\right)$ and $h^{d e r^{0}}{ }_{B, B^{\prime}}: \operatorname{Der}^{0}\left(\Lambda, \operatorname{Hom}_{R}\left(B, B^{\prime}\right)\right) \rightarrow$ $\operatorname{Der}^{0}\left(\Lambda, \operatorname{Hom}_{\bar{R}}\left(\bar{R} \otimes_{R} B, \bar{R} \otimes_{R} B^{\prime}\right)\right)$. They yield $\bar{R}$-homomorphisms $\breve{h}_{B, B^{\prime}}^{\text {der }}: \bar{R} \otimes_{R} \operatorname{Der}$ $\left(\Lambda, \operatorname{Hom}_{R}\left(B, B^{\prime}\right)\right) \rightarrow \operatorname{Der}\left(\Lambda, \operatorname{Hom}_{\bar{R}}\left(\bar{R} \otimes_{R} B, \bar{R} \otimes_{R} B^{\prime}\right)\right)$ and $\breve{h}^{\text {der }^{0}}{ }_{B, B^{\prime}}: \bar{R} \otimes_{R} \operatorname{Der}^{0}$ $\left(\Lambda, \operatorname{Hom}_{R}\left(B, B^{\prime}\right)\right) \rightarrow \operatorname{Der}^{0}\left(\Lambda, \operatorname{Hom}_{\bar{R}}\left(\bar{R} \otimes_{R} B, \bar{R} \otimes_{R} B^{\prime}\right)\right)$, which induce $\breve{h}^{\text {ext }}{ }_{B, B^{\prime}}$, if $B$ is in $\bmod _{R} \Lambda$ (cf. the commutative diagram in 4.10).

The last observation can be used for deciding whether $\breve{h^{e x t}}{ }_{B, B^{\prime}}$ is an isomorphism, in some situations. 
4.4

The following general result yields an answer to the question concerning the extension of the coefficient ring.

Theorem Let $\Lambda=k Q / I$ be a finite dimensional algebra of a bound quiver $(Q, I), R$ a commutative algebra, $B$ an $R \otimes_{k} \Lambda$-module from $\bmod _{R} \Lambda$ and $\mathcal{F}:=$ $\left\{F_{1}, \ldots, F_{\rho}\right\}$ an $R$-semisimple subcategory in $\bmod _{R} \Lambda$ such that for any $i=$ $1, \ldots, \rho$, we have an $R$-isomorphism $\operatorname{Ext}_{R \otimes \Lambda}^{1}\left(B, F_{i}\right) \cong R^{m_{i}}$, for some $m_{i} \in \mathbb{N}_{+}$. Assume that $h: R \rightarrow \bar{R}$ is a ring homomorphism such that the associated $R$ homomorphisms $\breve{h}^{\text {hom }}{ }_{F_{i}, F_{j}}: \bar{R} \otimes_{R} \operatorname{Hom}_{R \otimes \Lambda}\left(F_{i}, F_{j}\right) \rightarrow \operatorname{Hom}_{\bar{R} \otimes \Lambda}\left(\bar{F}_{i}, \bar{F}_{j}\right)$ and $\breve{h}_{B, F_{i}}^{\text {ext }}$ : $\bar{R} \otimes_{R} \operatorname{Ext}_{R \otimes \Lambda}^{1}\left(B, F_{i}\right) \rightarrow \operatorname{Ext}_{\bar{R} \otimes \Lambda}^{1}\left(\bar{B}, \bar{F}_{i}\right)$ are isomorphisms, for all $i, j=1, \ldots, \rho$, where $\bar{B}=\bar{R} \otimes_{R} B$ and $\bar{F}_{i}=\bar{R} \otimes_{R} F_{i}$. Then

$$
\Upsilon_{\overline{\mathcal{F}}}(\bar{B}) \cong \bar{R} \otimes_{R} \Upsilon_{\mathcal{F}}(B)
$$

in $\bmod _{\bar{R}} \Lambda$, where $\overline{\mathcal{F}}:=\left\{\bar{F}_{1}, \ldots \bar{F}_{\rho}\right\}$.

Note that under our assumptions, the existence of $\eta_{B}^{\mathcal{F}}$ and consequently of $\Upsilon_{\mathcal{F}}(B)$ is clear, moreover, $\overline{\mathcal{F}}$ is $\bar{R}$-semisimple and $\operatorname{Ext}_{\bar{R} \otimes \Lambda}^{1}\left(\bar{B}, \bar{F}_{i}\right) \cong \bar{R}^{m_{i}}$, for all $i$, so $\eta_{\bar{B}}^{\overline{\mathcal{F}}}$ and $\Upsilon_{\overline{\mathcal{F}}}(\bar{B})$ are well defined (see Theorem 4.1).

To prove the theorem (see Section 4.5 for the proof), we show that $\left[\eta_{\bar{B}}^{\overline{\mathcal{F}}}\right]=\left[\bar{\eta}_{B}^{\mathcal{F}}\right]$ under the identification

$$
\operatorname{Ext}_{R \otimes \Lambda}^{1}(\bar{B}, \ddot{\psi}): \operatorname{Ext}_{\bar{R} \otimes \Lambda}^{1}\left(\bar{B}, \bar{R} \otimes_{R} \Omega_{\mathcal{F}}(B)\right) \longrightarrow \operatorname{Ext}_{\bar{R} \otimes \Lambda}^{1}\left(\bar{B}, \Omega_{\overline{\mathcal{F}}}(\bar{B})\right)
$$

induced by some standard natural $\bar{R}$-isomorphism

$$
\ddot{\psi}: \bar{R} \otimes_{R} \Omega_{\mathcal{F}}(B) \longrightarrow \Omega_{\overline{\mathcal{F}}}(\bar{B})
$$

where

$$
\begin{aligned}
& \Omega_{\mathcal{F}}(B):=\bigoplus_{i=1}^{\rho} D^{R}\left(\operatorname{Ext}_{R \otimes \Lambda}^{1}\left(B, F_{i}\right)\right) \otimes_{R} F_{i}, \\
& \Omega_{\overline{\mathcal{F}}}(\bar{B}):=\bigoplus_{i=1}^{\rho} D^{\bar{R}}\left(\operatorname{Ext}_{\bar{R} \otimes \Lambda}^{1}\left(\bar{B}, \bar{F}_{i}\right)\right) \otimes_{\bar{R}} \bar{F}_{i}
\end{aligned}
$$

and $\bar{\eta}_{B}^{\mathcal{F}}:=\bar{R} \otimes_{R} \eta_{B}^{\mathcal{F}}$. More precisely, we show that

$$
\left[\ddot{\psi} \cdot \bar{\eta}_{B}^{\mathcal{F}}\right]=\left[\eta_{\bar{B}}^{\overline{\mathcal{F}}}\right]
$$

Notice that $\bar{R} \otimes_{R} \eta_{B}^{\mathcal{F}}$ is exact, since $\eta_{B}^{\mathcal{F}}$ splits over $R$. In consequence, there exists a canonical $\bar{R} \otimes_{k} \Lambda$-isomorphism $\hat{\ddot{\psi}}: \bar{R} \otimes_{R} \Upsilon_{\mathcal{F}}(B) \rightarrow \Upsilon_{\overline{\mathcal{F}}}(\bar{B})$ such that the diagram

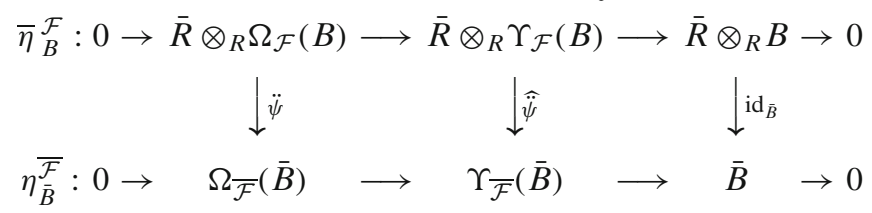

with exact rows, is commutative in $\bmod _{R} \Lambda$. 
The proof of the theorem needs some preparatory facts. They are mainly devoted to the construction of the map $\ddot{\psi}$.

Lemma Let $h: R \rightarrow \bar{R}$ be a commutative ring homomorphism and $E$ an $R$-module. Then there exists a canonical natural $\bar{R}$-homomorphism

$$
\psi=\psi_{E}: \bar{R} \otimes_{R} D^{R}(E) \longrightarrow D^{\bar{R}}\left(\bar{R} \otimes_{R} E\right)
$$

such that $\psi(s \otimes \sigma)\left(s^{\prime} \otimes e\right)=s s^{\prime} h(\sigma(e))$, for all $\sigma \in D^{R}(E), s, s^{\prime} \in \bar{R}$, and $e \in E$. Moreover, $\psi$ is an $\bar{R}$-isomorphism provided ${ }_{R} E$ is finitely generated free.

Proof An easy check on the definition.

Let $\Lambda=k Q / I$ be a finite dimensional $k$-algebra, where $(Q, I)$ is a bound quiver. Then for any $R \otimes_{k} \Lambda$-modules $X, Y$ in $\bmod _{R} \Lambda$ such that the $\bar{R}$-homomorphism

$$
\breve{h}_{X, Y}:=\breve{h}_{X, Y}^{\text {ext }}: \bar{R} \otimes_{R} \operatorname{Ext}_{R \otimes \Lambda}^{1}(X, Y) \rightarrow \operatorname{Ext}_{\bar{R} \otimes \Lambda}^{1}\left(\bar{R} \otimes_{R} X, \bar{R} \otimes_{R} Y\right)
$$

is an isomorphism, we denote by $\dot{\psi}_{X, Y}$ the composed $\bar{R}$-homomorphism

$$
\begin{aligned}
\bar{R} \otimes_{R} D^{R}\left(\operatorname{Ext}_{R \otimes \Lambda}^{1}(X, Y)\right) & \rightarrow D^{\bar{R}}\left(\bar{R} \otimes_{R} \operatorname{Ext}_{R \otimes \Lambda}^{1}(X, Y)\right) \\
& \rightarrow D^{\bar{R}}\left(\operatorname{Ext}_{\bar{R} \otimes \Lambda}^{1}\left(\bar{R} \otimes_{R} X, \bar{R} \otimes_{R} Y\right)\right)
\end{aligned}
$$

given by the formula

$$
\dot{\psi}_{X, Y}:=\operatorname{Hom}_{\bar{R}}\left(\left(\breve{h}_{X, Y}\right)^{-1}, \bar{R}\right) \circ \psi_{\operatorname{Ext}_{R \otimes \Lambda}^{1}(X, Y)}
$$

Proposition Let $\Lambda, h, X, Y$ and $\dot{\psi}_{X, Y}$ be as above.

(i) We have $\dot{\psi}_{X, Y}(s \otimes \sigma)\left(\breve{h}_{X, Y}\left(s^{\prime} \otimes e\right)\right)=s s^{\prime} h(\sigma(e))$, for all $s, s^{\prime} \in \bar{R}$, e $\in \operatorname{Ext}_{R \otimes \Lambda}^{1}$ $(X, Y)$ and $\sigma \in D^{R}\left(\operatorname{Ext}_{R \otimes \Lambda}^{1}(X, Y)\right)$,

(ii) If $\operatorname{Ext}_{R \otimes \Lambda}^{1}(X, Y)$ is a finitely generated free $R$-module then the homomorphism $\dot{\psi}_{X, Y}$ is an $\bar{R}$-isomorphism. Moreover, if $\mathcal{B}=\left\{e_{1}, \ldots, e_{m}\right\}$ is an $R$-basis of $\operatorname{Ext}_{R \otimes \Lambda}^{1}(X, Y)$ then $\overline{\mathcal{B}}:=\left\{\bar{e}_{1}, \ldots, \bar{e}_{m}\right\}$ is an $\bar{R}$-basis of $\operatorname{Ext}_{\bar{R} \otimes \Lambda}^{1}\left(\bar{R} \otimes_{R} X, \bar{R} \otimes_{R}\right.$ $Y)$, where $\bar{e}_{j}=\breve{h}_{X, Y}^{\text {ext }}\left(1 \otimes e_{j}\right)=h_{X, Y}^{\text {ext }}\left(e_{j}\right)$, and

$$
\dot{\psi}_{X, Y}\left(1 \otimes e_{j}^{R}\right)=\bar{e}_{j}^{\bar{R}}
$$

for any $j=1, \ldots, m$, where $e_{j}^{R} \in \mathcal{B}^{R}\left(\right.$ resp. $\left.\bar{e}_{j}^{\bar{R}} \in \overline{\mathcal{B}}^{\bar{R}}\right)$.

Proof The assertion (i) follows immediately from the lemma above. To show (ii) note that $\overline{\mathcal{B}}$ is an $\bar{R}$-basis, since $\breve{h}^{\text {ext }}{ }_{X, Y}$ is an $\bar{R}$-isomorphism. The main formula follows, since by (i) and the definition of $\overline{\mathcal{B}}$ we have the equalities

$$
\dot{\psi}_{X, Y}\left(1 \otimes e_{j}^{R}\right)\left(\bar{e}_{j^{\prime}}\right)=\dot{\psi}_{X, Y}\left(1 \otimes e_{j}^{R}\right)\left(\breve{h}_{X, Y}^{\text {ext }}\left(1 \otimes e_{j^{\prime}}\right)\right)=h\left(e_{j}^{R}\left(e_{j^{\prime}}\right)\right)=\bar{e}_{j}^{\bar{R}}\left(\bar{e}_{j^{\prime}}\right)
$$

for any pair $j, j^{\prime}=1, \ldots, m$. 
4.5

In the proof of Theorem 4.4 we use the fact that the extension groups behave nicely under the change of coefficients.

Lemma Let $h: R \rightarrow \bar{R}$ and $\Lambda$ be as above. Then for $R \otimes_{k} \Lambda$-modules $X, Y, Z$ such that $X$ belongs to $\bmod _{R} \Lambda$ and an $R \otimes_{k} \Lambda$-homomorphism $\phi: Y \rightarrow Z$ the diagram

$$
\begin{gathered}
h_{X, Y}^{\text {ext }}: \operatorname{Ext}_{R \otimes \Lambda}^{1}(X, Y) \longrightarrow \operatorname{Ext}_{\bar{R} \otimes \Lambda}^{1}\left(\bar{R} \otimes_{R} X, \bar{R} \otimes_{R} Y\right) \\
\qquad \operatorname{Ext}_{R \otimes \Lambda}^{1}(X, \phi) \\
h_{X, Z}^{\text {ext }}: \operatorname{Ext}_{R \otimes \Lambda}^{1}(X, Z) \longrightarrow \operatorname{Ext}_{\bar{R} \otimes \Lambda}^{1}(\bar{R} \otimes X, \bar{R} \otimes \phi) \\
\bar{R} \otimes \Lambda \\
1
\end{gathered}
$$

is a commutative square in $\bmod R$.

Proof Follows easily by applying the functor $\bar{R} \otimes_{R}-$ to the diagram defining the extension $\phi \cdot e$ induced by the map $\phi$, for $e \in \operatorname{Ext}_{R \otimes \Lambda}^{1}(X, Y)$. (Note that the respective maps and constructions are well defined, since $X$ belongs to $\bmod _{R} \Lambda$ ).

Proof of Theorem 4.4 We start by defining the announced $\bar{R} \otimes_{k} \Lambda$-isomorphism

$$
\ddot{\psi}: \bar{R} \otimes_{R}\left(\bigoplus_{i=1}^{\rho} D^{R}\left(\operatorname{Ext}_{R \otimes \Lambda}^{1}\left(B, F_{i}\right)\right) \otimes_{R} F_{i}\right) \longrightarrow \bigoplus_{i=1}^{\rho} D^{\bar{R}}\left(\operatorname{Ext}_{\bar{R} \otimes \Lambda}^{1}\left(\bar{B}, \bar{F}_{i}\right)\right) \otimes_{\bar{R}} \bar{F}_{i}
$$

setting $\ddot{\psi}:=\bigoplus_{i=1}^{\rho} \ddot{\psi}_{i}$, under the standard identification

$$
\bar{R} \otimes_{R}\left(\bigoplus_{i=1}^{\rho} D^{R}\left(\operatorname{Ext}_{R \otimes \Lambda}^{1}\left(B, F_{i}\right)\right) \otimes_{R} F_{i}\right)=\bigoplus_{i=1}^{\rho} \bar{R} \otimes_{R} D^{R}\left(\operatorname{Ext}_{R \otimes \Lambda}^{1}\left(B, F_{i}\right)\right) \otimes_{R} F_{i} .
$$

Each $\ddot{\psi}_{i}$, for $i=1, \ldots, \rho$, is a composed map

$$
\begin{aligned}
\bar{R} \otimes_{R} D^{R}\left(\operatorname{Ext}_{R \otimes \Lambda}^{1}\left(B, F_{i}\right)\right) \otimes_{R} F_{i} & \rightarrow D^{\bar{R}}\left(\operatorname{Ext}_{\bar{R} \otimes \Lambda}^{1}\left(\bar{B}, \bar{F}_{i}\right)\right) \otimes_{R} F_{i} \\
& \rightarrow D^{\bar{R}}\left(\operatorname{Ext}_{\bar{R} \otimes \Lambda}^{1}\left(\bar{B}, \bar{F}_{i}\right)\right) \otimes_{\bar{R}} \bar{R} \otimes_{R} F_{i}
\end{aligned}
$$

given by the formula

$$
\ddot{\psi}_{i}=\left(\kappa_{i}^{-1} \otimes \operatorname{id}_{F_{i}}\right) \circ\left(\dot{\psi}_{i} \otimes \mathrm{id}_{F_{i}}\right)
$$

where

$$
\dot{\psi}_{i}=\dot{\psi}_{B, F_{i}}: \bar{R} \otimes_{R} D^{R}\left(\operatorname{Ext}_{R \otimes \Lambda}^{1}\left(B, F_{i}\right)\right) \rightarrow D^{\bar{R}}\left(\operatorname{Ext}_{\bar{R} \otimes \Lambda}^{1}\left(\bar{B}, \bar{F}_{i}\right)\right)
$$

and

$$
\kappa_{i}: D^{\bar{R}}\left(\operatorname{Ext}_{\bar{R} \otimes \Lambda}^{1}\left(\bar{B}, \bar{F}_{i}\right)\right) \otimes_{\bar{R}} \bar{R} \rightarrow D^{\bar{R}}\left(\operatorname{Ext}_{\bar{R} \otimes \Lambda}^{1}\left(\bar{B}, \bar{F}_{i}\right)\right)
$$

is the standard $\bar{R}$-isomorphism (recall that $\bar{B}:=\bar{R} \otimes_{R} B$ and $\bar{F}_{i}:=\bar{R} \otimes_{R} F_{i}$ ). It is clear that each $\ddot{\psi}_{i}$ is an $\bar{R} \otimes_{R} \Lambda$-isomorphism, since $\dot{\psi}_{i}$ and $\kappa_{i}$ are $\bar{R}$-isomorphisms (see 
Proposition 4.4(ii)). Moreover, for any $s \in \bar{R}, \sigma \in D^{R}\left(\operatorname{Ext}_{R \otimes \Lambda}^{1}\left(B, F_{i}\right)\right)$ and $f \in F_{i}$, we have

$$
\ddot{\psi}_{i}(s \otimes \sigma \otimes f)=s \dot{\psi}_{i}(1 \otimes \sigma) \otimes 1 \otimes f=\dot{\psi}_{i}(1 \otimes \sigma) \otimes s \otimes f .
$$

As we mentioned before to prove the assertion it suffices to show that

$$
\left[\ddot{\psi} \cdot \bar{\eta}_{B}^{\mathcal{F}}\right]=\left[\eta_{\bar{B}}^{\overline{\mathcal{F}}}\right]
$$

where $\bar{\eta}_{B}^{\mathcal{F}}:=\bar{R} \otimes_{R} \eta_{B}^{\mathcal{F}}$. For this aim we fix a collection $\mathcal{E}$ of $R$-bases $\mathcal{E}_{i}:=\left\{e_{j}^{(i)}\right\}_{j=1, \ldots, m_{i}}$ of the finitely generated free $R$-modules $\operatorname{Ext}_{R \otimes \Lambda}^{1}\left(B, F_{i}\right)$, for $i=1, \ldots, \rho$. Then we have the diagonal $R \otimes_{k} \Lambda$-homomorphism $\varepsilon=\varepsilon(\mathcal{E})$ as in Theorem 4.1,

$$
\varepsilon:=\bigoplus_{i=1}^{\rho} \varepsilon_{i}: \bigoplus_{i=1}^{\rho} \bigoplus_{j=1}^{m_{i}} F_{i} \longrightarrow \bigoplus_{i=1}^{\rho} D^{R}\left(\mathrm{Ext}_{R \otimes \Lambda}^{1}\left(B, F_{i}\right)\right) \otimes_{R} F_{i}
$$

where each $\varepsilon_{i}:=\left[\left(e_{j}^{(i)}\right)^{R} \otimes-\right]_{j}: \bigoplus_{j=1}^{m_{i}} F_{i} \longrightarrow D^{R}\left(\operatorname{Ext}_{R \otimes \Lambda}^{1}\left(B, F_{i}\right)\right) \otimes_{R} F_{i}$, for $i=$ $1, \ldots, \rho$ is a row matrix map; moreover, $\left[\eta_{B}^{\mathcal{F}}\right]=\varepsilon \cdot e$, where $e=\left(e_{j}^{(i)}\right)_{i, j} \in$ $\operatorname{Ext}_{R \otimes \Lambda}^{1}(B, F) \quad\left(=\bigoplus_{i=1}^{\rho} \bigoplus_{j=1}^{m_{i}} \operatorname{Ext}_{R \otimes \Lambda}^{1}\left(B, F_{i}\right)\right)$, for $F:=\bigoplus_{i=1}^{\rho} \bigoplus_{j=1}^{m_{i}} F_{i}$.

For any $i=1, \ldots, \rho$, we denote by $\overline{\mathcal{E}}_{i}$ the image $h^{\text {ext }}{ }_{B, F_{i}}\left(\mathcal{E}_{i}\right)=\left\{\bar{e}_{j}^{(i)}\right\}_{j=1, \ldots, m_{i}}$ $\left(\subseteq \operatorname{Ext}_{\bar{R} \otimes \Lambda}^{1}\left(\bar{B}, \bar{F}_{i}\right)\right)$, where $\bar{e}_{j}^{(i)}=h^{\text {ext }}{ }_{B, F_{i}}\left(e_{j}^{(i)}\right)$ for every $j=1, \ldots, m_{i}$. Then by Lemma 4.5 we have

$$
h_{B, \Omega_{\mathcal{F}}(B)}^{\text {ext }} \circ \operatorname{Ext}_{R \otimes \Lambda}^{1}(B, \varepsilon)=\operatorname{Ext}_{\bar{R} \otimes \Lambda}^{1}(\bar{B}, \bar{R} \otimes \varepsilon) \circ h^{\text {ext }}{ }_{B, F} .
$$

Consequently,

$$
\left[\bar{\eta}_{B}^{\mathcal{F}}\right]=(\bar{R} \otimes \varepsilon) \cdot \bar{e} \quad\left(\in \operatorname{Ext}_{\bar{R} \otimes \Lambda}^{1}\left(\bar{B}, \bar{R} \otimes_{R} \Omega_{\mathcal{F}}(B)\right)\right)
$$

where $\bar{e}=\left(\bar{e}_{j}^{(i)}\right)_{i, j} \in \operatorname{Ext}_{\bar{R} \otimes \Lambda}^{1}\left(\bar{B}, \bar{R} \otimes_{R} F\right) \quad\left(=\bigoplus_{i=1}^{\rho} \bigoplus_{j=1}^{m_{i}} \operatorname{Ext}_{\bar{R} \otimes \Lambda}^{1}\left(\bar{B}, \bar{F}_{i}\right)\right)$, since

$$
\left[\bar{\eta}_{B}^{\mathcal{F}}\right]=h^{\text {ext }}{ }_{B, \Omega_{\mathcal{F}}(B)}\left(\left[\eta_{B}^{\mathcal{F}}\right]\right)=h_{B, \Omega_{\mathcal{F}}(B)}^{\text {ext }}(\varepsilon \cdot e)
$$

and

$$
h^{e x t}{ }_{B, F}(e)=\bar{e} .
$$

On the other hand, observe that by our assumptions, $\operatorname{Ext}_{\bar{R} \otimes \Lambda}^{1}\left(\bar{B}, \bar{F}_{i}\right)$ is a free finitely generated $\bar{R}$-module of rank $m_{i}$ and $\overline{\mathcal{E}}_{i}$ forms an $\bar{R}$-basis of $\operatorname{Ext}_{\bar{R} \otimes \Lambda}^{1}\left(\bar{B}, \bar{F}_{i}\right)$, for any $i=1, \ldots, \rho$ (see Proposition 4.4(ii)). Analogously as before, consider the $\bar{R} \otimes_{k} \Lambda$-homomorphism $\overline{\mathcal{E}}:=\varepsilon(\overline{\mathcal{E}})$, for $\overline{\mathcal{E}}:=\left\{\overline{\mathcal{E}}_{i}\right\}_{i=1, \ldots, \rho}$,

$$
\bar{\varepsilon}:=\bigoplus_{i=1}^{\rho} \bar{\varepsilon}_{i}: \bigoplus_{i=1}^{\rho} \bigoplus_{j=1}^{m_{i}} \bar{F}_{i} \longrightarrow \bigoplus_{i=1}^{\rho} D^{\bar{R}}\left(\operatorname{Ext}_{\bar{R} \otimes \Lambda}^{1}\left(\bar{B}, \bar{F}_{i}\right)\right) \otimes_{\bar{R}} \bar{F}_{i}
$$

where $\bar{\varepsilon}_{i}$, for $i=1, \ldots, \rho$, is a row matrix map $\left[\left(\bar{e}_{j}^{(i)}\right)^{\bar{R}} \otimes-\right]_{j}: \bigoplus_{j=1}^{m_{i}} \bar{F}_{i} \longrightarrow$ $D^{\bar{R}}\left(\operatorname{Ext}_{\bar{R} \otimes \Lambda}^{1}\left(\bar{B}, \bar{F}_{i}\right)\right) \otimes_{\bar{R}} \bar{F}_{i}$. Note that we have $\left[\eta_{\bar{B}}^{\overline{\mathcal{F}}}\right]=\bar{\varepsilon} \cdot \bar{e}$ (see Theorem 4.1).

Now to prove $(*)$ it suffices to show the equality

$$
\ddot{\psi} \circ(\bar{R} \otimes \varepsilon)=\bar{\varepsilon}
$$


under the standard identification $\bar{R} \otimes_{R} F=\bigoplus_{i=1}^{\rho} \bigoplus_{j=1}^{m_{i}} \bar{F}_{i}$, or equivalently the equalities

$$
\ddot{\psi}_{i} \circ\left(\bar{R} \otimes \varepsilon_{i}\right)=\bar{\varepsilon}_{i}
$$

for every $i=1, \ldots, \rho$, since (ii) and (**) imply

$$
\ddot{\psi} \cdot\left[\bar{\eta}_{B}^{\mathcal{F}}\right]=\ddot{\psi} \cdot((\bar{R} \otimes \varepsilon) \cdot \bar{e})=(\ddot{\psi} \circ(\bar{R} \otimes \varepsilon)) \cdot \bar{e}=\bar{\varepsilon} \cdot \bar{e}=\left[\eta_{\bar{B}}^{\overline{\mathcal{F}}}\right]
$$

Now the formula $(* *)_{i}$, for a fixed $i=1, \ldots, \rho$, is almost straightforward, since by (i), Proposition 4.4(ii) and the definition of $\bar{\varepsilon}_{i}$, for any $\left(s_{j} \otimes f_{j}\right)_{j} \in \bigoplus_{j=1}^{m_{i}} \bar{F}_{i}$ $\left(=\bigoplus_{j=1}^{m_{i}} \bar{R} \otimes_{R} F_{i}\right)$ we have

$$
\begin{aligned}
\ddot{\psi}_{i}\left(\left(\bar{R} \otimes \varepsilon_{i}\right)\left(\left(s_{j} \otimes f_{j}\right)_{j}\right)\right. & =\ddot{\psi}_{i}\left(\sum_{j=1}^{m_{i}} s_{j} \otimes\left(e_{j}^{(i)}\right)^{R} \otimes f_{j}\right) \\
& =\sum_{j=1}^{m_{i}} \dot{\psi}_{i}\left(1 \otimes\left(e_{j}^{(i)}\right)^{R}\right) \otimes s_{j} \otimes f_{j} \\
& =\sum_{j=1}^{m_{i}}\left(\bar{e}_{j}^{(i)}\right)^{\bar{R}} \otimes\left(s_{j} \otimes f_{j}\right)=\bar{\varepsilon}\left(\left(s_{j} \otimes f_{j}\right)_{j}\right) .
\end{aligned}
$$

In this way the proof of $(* *)$ and $(*)$ is complete. Consequently, the theorem is proved.

4.6

The next result describes the behaviour of universal extensions of bimodules under the "restriction of coefficients" to the ground field $k$, in some specific situation.

Theorem Let $\Lambda=k Q /$ I be a finite dimensional algebra of a bound quiver $(Q, I), A$ a commutative finite dimensional selfinjective $k$-algebra, $C$ an $A \otimes_{k} \Lambda$-module from $\bmod _{A} \Lambda$ and $\mathcal{F}:=\left\{F_{1}, \ldots, F_{\rho}\right\}$ a semisimple subcategory in $\bmod \Lambda$. Assume that for any $i=1, \ldots, \rho$, we have an $A$-isomorphism $\operatorname{Ext}_{A \otimes \Lambda}^{1}\left(C, \bar{F}_{i}\right) \cong A^{m_{i}}$, for some $m_{i} \in$ $\mathbb{N}_{+}$, where $\bar{F}_{i}:=A \otimes_{k} F_{i}$. Then

$$
\Upsilon_{\overline{\mathcal{F}}}(C)_{\Lambda} \cong \Upsilon_{\mathcal{F}}\left(C_{\Lambda}\right)
$$

in $\bmod \Lambda$, where $\overline{\mathcal{F}}:=\left\{\bar{F}_{1}, \ldots \bar{F}_{\rho}\right\}$.

Note that by Lemma 4.3, $\overline{\mathcal{F}}$ is $A$-semisimple, since $\mathcal{F}$ is semisimple, so $\Upsilon_{\overline{\mathcal{F}}}(C)$ is well defined (see Theorem 4.1). Clearly, the same holds true for $\Upsilon_{\mathcal{F}}(C):=\Upsilon_{\mathcal{F}}\left(C_{\Lambda}\right)$.

To prove the theorem (see Section 4.7 for the proof), we show that $\left[\eta_{C}^{\overline{\mathcal{F}}}\right]=\left[\eta_{C}^{\mathcal{F}}\right]$ under the identification

$$
\operatorname{Ext}^{1}(C, \ddot{\varphi}): \operatorname{Ext}_{A \otimes \Lambda}^{1}\left(C, \Omega_{\overline{\mathcal{F}}}(C)\right) \longrightarrow \operatorname{Ext}_{A \otimes \Lambda}^{1}\left(C, \Omega_{\mathcal{F}}(C)\right)
$$

induced by some standard natural $A$-isomorphism

$$
\ddot{\varphi}: \Omega_{\mathcal{F}}(C) \longrightarrow \Omega_{\mathcal{F}}(C)
$$


where

$$
\Omega_{\overline{\mathcal{F}}}(C):=\bigoplus_{i=1}^{\rho} D^{A}\left(\operatorname{Ext}_{A \otimes \Lambda}^{1}\left(C, \bar{F}_{i}\right)\right) \otimes_{A} \bar{F}_{i}
$$

and

$$
\Omega_{\mathcal{F}}(C):=\bigoplus_{i=1}^{\rho} D\left(\operatorname{Ext}_{\Lambda}^{1}\left(C, F_{i}\right)\right) \otimes_{k} F_{i}
$$

(In fact we set here $\eta_{C}^{\mathcal{F}}:=\eta_{C_{\Lambda}}^{\mathcal{F}}$ and $\Omega_{\mathcal{F}}(C):=\Omega_{\mathcal{F}}\left(C_{\Lambda}\right)$ ). More precisely, we show that

$$
\left[\ddot{\varphi} \cdot \eta_{C}^{\overline{\mathcal{F}}}\right]=\left[\eta_{C}^{\mathcal{F}}\right]
$$

(see the remark below). In consequence, there exists a $\Lambda$-isomorphism $\hat{\ddot{\varphi}}: \Upsilon_{\overline{\mathcal{F}}}(C) \rightarrow$ $\Upsilon_{\mathcal{F}}(C)$ such that the diagram

$$
\begin{aligned}
& \eta_{C}^{\overline{\mathcal{F}}}: \quad 0 \rightarrow \Omega_{\overline{\mathcal{F}}}(C) \longrightarrow \Upsilon_{\overline{\mathcal{F}}}(C) \longrightarrow C \rightarrow 0 \\
& \eta_{C}^{\mathcal{F}}: \quad 0 \rightarrow \Omega_{\mathcal{F}}(C) \longrightarrow \Upsilon_{\mathcal{F}}(C) \longrightarrow C \rightarrow 0
\end{aligned}
$$

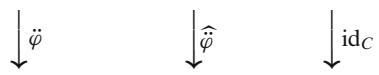

with exact rows, is commutative in $\bmod \Lambda$.

Remark Let ${ }_{A} X_{\Lambda},{ }_{A} Y_{\Lambda}$ be $A$ - $\Lambda$-bimodules and $Z_{\Lambda}$ be a $\Lambda$-module. Then a $\Lambda$-homomorphism $\phi: Y \rightarrow Z$ induces an $A$-homomorphism $\operatorname{Ext}^{1}(X, \phi)$ : $\operatorname{Ext}_{A \otimes \Lambda}^{1}(X, Y) \rightarrow \operatorname{Ext}_{\Lambda}^{1}(X, Z)$ which is defined as a composed map

$$
\operatorname{Ext}_{A \otimes \Lambda}^{1}(X, Y) \longrightarrow \operatorname{Ext}_{\Lambda}^{1}(X, Y) \stackrel{\operatorname{Ext}_{\Lambda}^{1}(X, \phi)}{\longrightarrow} \operatorname{Ext}_{\Lambda}^{1}(X, Z)
$$

and is given by the mapping $[v] \mapsto[\phi \cdot v]$, for $[v] \in \operatorname{Ext}_{A \otimes \Lambda}^{1}(X, Y)$.

The main proof needs some preparatory facts, necessary mainly for the construction of $\ddot{\varphi}$. To formulate our first result we fix a notation. For any $\gamma \in A^{*}=D(A)$ we denote by $\tilde{\gamma}=\tilde{\gamma}^{M}$ the composed $k$-homomorphism

$$
A \otimes_{k} M \stackrel{\gamma \otimes \text { id }}{\longrightarrow} k \otimes_{k} M \stackrel{\cong}{\longrightarrow} M
$$

where $M$ is a $k$-space.

Lemma Let $A$ be a commutative selfinjective finite dimensional $k$-algebra and $\theta=$ $\theta_{(-)}: A \rightarrow A^{*}$ a fixed $A$-isomorphism. Then for any $A$-module $P$ and $k$-space $M$ there exists a canonical, natural with respect to both coordinates, $A$-isomorphism

$$
\varphi=\varphi_{P, M}: \operatorname{Hom}_{A}\left(P, A \otimes_{k} M\right) \rightarrow \operatorname{Hom}_{k}(P, M)
$$

dependent on $\theta$, satisfying the following conditions:

(i) $\varphi(g)=\sum_{i \in I} \theta_{(1)}\left(g_{i}(-)\right) \cdot b_{i}$, for $g \in \operatorname{Hom}_{A}\left(P, A \otimes_{k} M\right)$, where $\mathcal{B}=\left\{b_{i}\right\}_{i \in I}$ is an arbitrary $k$-basis of $M$ and $g=\sum_{i \in I} g_{i}(-) \otimes b_{i}$ is a canonical presentation of $g$ with respect to $\mathcal{B}$,

(ii) $a \varphi(g)=\widetilde{\theta_{(a)}} \circ g$, for any $a \in A$ and $g \in \operatorname{Hom}_{A}\left(P, A \otimes_{k} M\right)$. 
Proof Denote by $\varphi=\varphi_{P, M}$ the composed $A$-isomorphism

$$
\begin{aligned}
\operatorname{Hom}_{A}\left(P, A \otimes_{k} M\right) & \cong \operatorname{Hom}_{A}\left(P, \operatorname{Hom}_{k}\left(A^{*}, M\right)\right) \\
& \cong \operatorname{Hom}_{k}\left(A^{*} \otimes_{A} P, M\right) \cong \operatorname{Hom}_{k}\left(A \otimes_{A} P, M\right) \\
& \cong \operatorname{Hom}_{k}(P, M)
\end{aligned}
$$

where the first isomorphism is induced by the standard $A$-isomorphism $A \otimes_{k} M \cong$ $\operatorname{Hom}_{k}\left(A^{*}, M\right)$, the second one is given by the respective adjointness formula, the third one is induced by $\theta: A \rightarrow A^{*}$ and the forth one by the canonical $A$ isomorphism $P \cong A \otimes_{A} P$.

Take $g \in \operatorname{Hom}_{A}\left(P, A \otimes_{k} M\right)$. Then for any $p \in P$ we have $g(p)=\sum_{i \in I} g_{i}(p) \otimes b_{i}$, where $\mathcal{B}=\left\{b_{i}\right\}_{i \in I}$ is a fixed $k$-basis of $M$. (Note that $g_{i}(p)=0$ for almost all $i \in I$ and that $g_{i} \in \operatorname{Hom}_{A}(P, A)$ for all $\left.i \in I\right)$. Denote by $g^{(1)}, g^{(2)}, g^{(3)}, g^{(4)}=\varphi(g)$ the images of $g$ via consecutive "left factors" of $\varphi$. Then for $p \in P$ and $\gamma \in A^{*}$ we have $g^{(2)}(\gamma \otimes$ $p)=g^{(1)}(p)(\gamma)=\sum_{i \in I} \gamma\left(g_{i}(p)\right) \cdot b_{i}$, hence

$$
\varphi(g)(p)=g^{(3)}(1 \otimes p)=g^{(2)}\left(\theta_{(1)} \otimes p\right)=\sum_{i \in I} \theta_{(1)}\left(g_{i}(p)\right) \cdot b_{i}
$$

and (i) is proved.

To show (ii) observe that for $a \in A, g \in \operatorname{Hom}_{A}\left(P, A \otimes_{k} M\right)$ and $p \in P$ we have

$$
\begin{aligned}
(a \varphi(g))(p) & =\sum_{i \in I} \theta_{(1)}\left(g_{i}(a p)\right) \cdot b_{i}=\sum_{i \in I} \theta_{(1)}\left(a g_{i}(p)\right) \cdot b_{i} \\
& =\sum_{i \in I}\left(a \theta_{(1)}\right)\left(g_{i}(p)\right) \cdot b_{i}=\sum_{i \in I} \theta_{(a)}\left(g_{i}(p)\right) b_{i}
\end{aligned}
$$

and moreover,

$$
\left(\widetilde{\theta_{(a)}} \circ g\right)(p)=\widetilde{\theta_{(a)}}\left(\sum_{i \in I} g_{i}(p) \otimes b_{i}\right)=\sum_{i \in I} \theta_{(a)}\left(g_{i}(p)\right) \cdot b_{i}
$$

Proposition Let $A, \theta$ be as above and $\Lambda=k Q / I$ be a finite dimensional algebra, where $(Q, I)$ is a bound quiver. Then for any $A \otimes \Lambda$-module $P$ and $\Lambda$-module $M$ the map

$$
\bigoplus_{\alpha \in Q_{1}} \varphi_{P_{s(\alpha)}, M_{t(\alpha)}}: \bigoplus_{\alpha \in Q_{1}} \operatorname{Hom}_{A}\left(P_{s(\alpha)}, A \otimes_{k} M_{t(\alpha)}\right) \rightarrow \bigoplus_{\alpha \in Q_{1}} \operatorname{Hom}_{k}\left(P_{s(\alpha)}, M_{t(\alpha)}\right)
$$

induces A-isomorphisms

$$
\varphi_{P, M}:=\varphi_{P, M}^{d e r}: \operatorname{Der}\left(\Lambda, \operatorname{Hom}_{A}\left(P, A \otimes_{k} M\right)\right) \longrightarrow \operatorname{Der}\left(\Lambda, \operatorname{Hom}_{k}(P, M)\right)
$$

respectively, $\varphi_{P, M}^{d e r^{0}}: \operatorname{Der}^{0}\left(\Lambda, \operatorname{Hom}_{A}\left(P, A \otimes_{k} M\right)\right) \longrightarrow \operatorname{Der}^{0}\left(\Lambda, \operatorname{Hom}_{k}(P, M)\right)$ such that

$$
a \varphi_{P, M}(\delta)=\widetilde{\theta_{(a)}} \circ \delta
$$


for any $a \in A$ and $\delta=\left(\delta_{\alpha}\right)_{\alpha \in Q_{1}} \in \operatorname{Der}\left(\Lambda, \operatorname{Hom}_{A}\left(P, A \otimes_{k} M\right)\right)$, where $\widetilde{\theta_{(a)}}:=$ $\left(\widetilde{\theta_{(a)}}{ }^{M_{t(\alpha)}}\right)_{\alpha \in Q_{1}}$ and $\widetilde{\theta_{(a)}} \circ \delta=\left(\widetilde{\theta_{(a)}}{ }^{M_{t(\alpha)}} \circ \delta_{\alpha}\right)_{\alpha}$. In particular, if P belongs to $\bmod _{A} \Lambda$ then also the A-isomorphism

$$
\varphi_{P, M}:=\varphi_{P, M}^{e x t}: \operatorname{Ext}_{A \otimes \Lambda}^{1}\left(P, A \otimes_{k} M\right) \longrightarrow \operatorname{Ext}_{\Lambda}^{1}(P, M)
$$

such that

$$
a \varphi_{P, M}(e)=\widetilde{\theta_{(a)}} \cdot e
$$

for any $a \in A$ and $e \in \operatorname{Ext}_{A \otimes \Lambda}^{1}\left(P, A \otimes_{k} M\right)$, where $\widetilde{\theta_{(a)}}:=\left(\widetilde{\theta_{(a)}}{ }^{M_{x}}\right)_{x \in Q_{0}}$.

Proof The maps $\varphi_{P, M}^{d e r}$ and $\varphi_{P, M}^{d e 0^{0}}$ are well defined and are $A$-isomorphisms, since the isomorphism $\varphi$ from the lemma is natural with respect to both coordinates. Then the formula in the assertion is an immediate consequence of that from Lemma 4.6(ii). The assertions concerning $\varphi_{P, M}^{e x t}$ follow from the previous, by Lemma 4.2.

\section{7}

Let $A, \theta$ be as above. Then for any $A$-module $N$ there exists a canonical natural $A$-isomorphism

$$
\dot{\theta}_{N}: D^{A}(N) \rightarrow D(N)
$$

defined by the sequence of $A$-isomorphisms

$$
\operatorname{Hom}_{A}(N, A) \cong \operatorname{Hom}_{A}\left(N, A^{*}\right) \cong \operatorname{Hom}_{k}\left(A \otimes_{A} N, k\right) \cong \operatorname{Hom}_{k}(N, k)
$$

where the first one is given by $\operatorname{Hom}_{A}(N, \theta)$, the second by the adjunction formula and the third is induced by the canonical isomorphism $N \cong A \otimes_{A} N$. It is easily seen that

$$
\dot{\theta}(\sigma)=\theta_{(1)} \circ \sigma
$$

for $\sigma \in D^{A}(N)$.

For $P, M$ and $\Lambda$ as in the Proposition 4.6 we denote by $\dot{\varphi}$ the composed $A$ homomorphism

$$
\dot{\varphi}_{P, M}: D^{A}\left(\operatorname{Ext}_{A \otimes \Lambda}^{1}\left(P, A \otimes_{k} M\right)\right) \rightarrow D^{A}\left(\operatorname{Ext}_{\Lambda}^{1}(P, M)\right) \rightarrow D\left(\operatorname{Ext}_{\Lambda}^{1}(P, M)\right)
$$

given by the formula

$$
\dot{\varphi}_{P, M}:=\dot{\theta}_{\operatorname{Ext}_{\Lambda}^{1}(P, M)} \circ \operatorname{Hom}_{A}\left(\varphi_{P, M}^{-1}, A\right) .
$$

\section{Lemma}

(i) $\dot{\varphi}_{P, M}$ is an A-isomorphism.

(ii) $\dot{\varphi}_{P, M}(\sigma)=\theta_{(1)} \circ \sigma \circ \varphi_{P, M}^{-1}$, for any $\sigma \in D^{A}\left(\operatorname{Ext}_{A \otimes \Lambda}^{1}\left(P, A \otimes_{k} M\right)\right)$.

Proof Follows from Proposition 4.6 and the definition of $\dot{\varphi}$. 
Proof of Theorem 4.6 We start by defining the $A$-homomorphism

$$
\ddot{\varphi}: \bigoplus_{i=1}^{\rho} D^{A}\left(\operatorname{Ext}_{A \otimes \Lambda}^{1}\left(C, \bar{F}_{i}\right)\right) \otimes_{A} \bar{F}_{i} \longrightarrow \bigoplus_{i=1}^{\rho} D\left(\operatorname{Ext}_{\Lambda}^{1}\left(C, F_{i}\right)\right) \otimes_{k} F_{i}
$$

(see Section 4.6), setting $\ddot{\varphi}:=\bigoplus_{i=1}^{\rho} \ddot{\varphi}_{i}$. Each $\ddot{\varphi}_{i}$, for $i=1, \ldots, \rho$, is the composed map $\ddot{\varphi}_{i}: D^{A}\left(\operatorname{Ext}_{A \otimes \Lambda}^{1}\left(C, \bar{F}_{i}\right)\right) \otimes_{A} \bar{F}_{i} \longrightarrow D\left(\operatorname{Ext}_{\Lambda}^{1}\left(C, F_{i}\right)\right) \otimes_{A} \bar{F}_{i} \longrightarrow D\left(\operatorname{Ext}_{\Lambda}^{1}\left(C, F_{i}\right)\right) \otimes_{k} F_{i}$ given by the formula

$$
\ddot{\varphi}_{i}:=\left(\kappa_{i} \otimes \operatorname{id}_{F_{i}}\right) \circ\left(\dot{\varphi}_{i} \otimes \operatorname{id}_{\bar{F}_{i}}\right)
$$

where

$$
\dot{\varphi}_{i}:=\dot{\varphi}_{C, F_{i}}: D^{A}\left(\operatorname{Ext}_{A \otimes \Lambda}^{1}\left(C, \bar{F}_{i}\right)\right) \longrightarrow D\left(\operatorname{Ext}_{\Lambda}^{1}\left(C, F_{i}\right)\right)
$$

and

$$
\kappa_{i}: D\left(\operatorname{Ext}_{\Lambda}^{1}\left(C, F_{i}\right)\right) \otimes_{A} A \longrightarrow D\left(\operatorname{Ext}_{\Lambda}^{1}\left(C, F_{i}\right)\right)
$$

is the standard $A$-isomorphism $\left(\bar{F}_{i}=A \otimes_{k} F_{i} !\right)$. It is clear that each $\ddot{\varphi}_{i}$ is a $\Lambda$ isomorphism (in fact $A \otimes \Lambda$-isomorphism), since $\dot{\varphi}_{i}$ and $\kappa_{i}$ are $A$-isomorphisms (see Lemma 4.7(i)). Moreover, for $\sigma \in D^{A}\left(\operatorname{Ext}_{A \otimes \Lambda}^{1}\left(C, \bar{F}_{i}\right)\right), a \in A$ and $f \in F_{i}$, we have

$$
\ddot{\varphi}_{i}(\sigma \otimes a \otimes f)=\left(\theta_{(a)} \circ \sigma \circ \varphi_{i}^{-1}\right) \otimes f
$$

where $\varphi_{i}=\varphi_{C, F_{i}}$, since by Lemma 4.7(ii)

$$
\left(\dot{\varphi}_{i} \otimes \operatorname{id}_{\bar{F}_{i}}\right)(\sigma \otimes a \otimes f)=\left(\theta_{(1)} \circ \sigma \circ \varphi_{i}^{-1}\right) \otimes a \otimes f
$$

and applying $\left(\kappa_{i} \otimes \mathrm{id}_{F_{i}}\right)$ we obtain

$$
\begin{aligned}
\ddot{\varphi}_{i}(\sigma \otimes a \otimes f) & =a\left(\theta_{(1)} \circ \sigma \circ \varphi_{i}^{-1}\right) \otimes f=\left(\theta_{(1)} \circ \sigma \circ \varphi_{i}^{-1}(a-)\right) \otimes f \\
& =\left(\theta_{(1)}\left(a \sigma \circ \varphi_{i}^{-1}(-)\right)\right) \otimes f=\left(\left(a \theta_{(1)}\right) \circ \sigma \circ \varphi_{i}^{-1}(-)\right) \otimes f \\
& =\left(\theta_{(a)} \circ \sigma \circ \varphi_{i}^{-1}\right) \otimes f .
\end{aligned}
$$

As we mentioned before to prove the assertion it suffices to show that

$$
\left[\ddot{\varphi} \cdot \eta_{C}^{\overline{\mathcal{F}}}\right]=\left[\eta_{C}^{\mathcal{F}}\right]
$$

For this aim we fix a collection $\mathcal{E}$ of $A$-bases $\mathcal{E}_{i}:=\left\{e_{j}^{(i)}\right\}_{j=1, \ldots, m_{i}}$ of the finitely generated free $A$-modules $\operatorname{Ext}_{A \otimes \Lambda}^{1}\left(C, \bar{F}_{i}\right)$, for $i=1, \ldots, \rho$. $\mathcal{E}$ yields the diagonal $A \otimes_{k} \Lambda$-homomorphism $\varepsilon=\varepsilon(\mathcal{E})$,

$$
\varepsilon:=\bigoplus_{i=1}^{\rho} \varepsilon_{i}: \bigoplus_{i=1}^{\rho} \bigoplus_{j=1}^{m_{i}} \bar{F}_{i} \longrightarrow \bigoplus_{i=1}^{\rho} D^{A}\left(\mathrm{Ext}_{A \otimes \Lambda}^{1}\left(C, \bar{F}_{i}\right)\right) \otimes_{A} \bar{F}_{i},
$$

as in Theorem 4.1, where each $\varepsilon_{i}:=\left[\left(e_{j}^{(i)}\right)^{A} \otimes-\right]_{j}: \bigoplus_{j=1}^{m_{i}} \bar{F}_{i} \longrightarrow D^{A}\left(\mathrm{Ext}_{A \otimes \Lambda}^{1}\right.$ $\left.\left(C, \bar{F}_{i}\right)\right) \otimes_{A} \bar{F}_{i}$, for $i=1, \ldots, \rho$, is the map whose $j$ th component is given by the mapping $a \otimes f \mapsto\left(e_{j}^{(i)}\right)^{A} \otimes a \otimes f$, for $a \otimes f \in \bar{F}_{i}=A \otimes_{k} F_{i}$. 
We also fix a $k$-basis $\mathcal{B}=\left\{b_{u}\right\}_{u=1, \ldots, l}$ of $A$, where $l=\operatorname{dim}_{k} A$. For any $i=1, \ldots, \rho$ and $u=1, \ldots, l$, we denote by $\pi_{u}^{(i)}$ the $k$-homomorphism

$$
\pi_{u}^{(i)}:=\widetilde{b_{u}^{*}}: A \otimes_{k} F_{i} \rightarrow F_{i}
$$

which is in fact $\Lambda$-homomorphism, where $b_{u}^{*} \in \mathcal{B}^{*}$ (see Section 4.1), and for any $j=$ $1, \ldots, m_{i}$, by $e_{j, u}^{(i)}$ the element

$$
e_{j, u}^{(i)}:=\pi_{u}^{(i)} \cdot e_{j}^{(i)}=\operatorname{Ext}^{1}\left(C, \pi_{u}^{(i)}\right)\left(e_{j}^{(i)}\right) \in \operatorname{Ext}_{\Lambda}^{1}\left(C, F_{i}\right) .
$$

For any $i=1, \ldots, \rho$, we denote by $\pi^{(i)}$ the column matrix map

$$
\left[\pi_{u}^{(i)}\right]_{u}: \bar{F}_{i} \rightarrow \bigoplus_{u=1}^{l} F_{i}
$$

and we set

$$
\pi:=\bigoplus_{i=1}^{\rho} \bigoplus_{j=1}^{m_{i}} \pi^{(i)}: \bigoplus_{i=1}^{\rho} \bigoplus_{j=1}^{m_{i}} \bar{F}_{i} \longrightarrow \bigoplus_{i=1}^{\rho} \bigoplus_{j=1}^{m_{i}}\left(\bigoplus_{u=1}^{l} F_{i}\right) .
$$

The proof of $(*)$ reduces to two claims. First we show that

$1^{o} \quad$ The set $\mathcal{E}_{i}^{\prime}:=\left\{e_{j, u}^{(i)}\right\}_{j=1, \ldots, m_{i} ; u=1, \ldots, l}$ forms a $k$-basis of the space $\operatorname{Ext}_{\Lambda}^{1}\left(C, F_{i}\right)$, for every $i=1, \ldots, \rho$.

Assume that $1^{o}$ is already proved. Then we can define the map

$$
\varepsilon^{\prime}=\bigoplus_{i=1}^{\rho} \varepsilon_{i}^{\prime}: \bigoplus_{i=1}^{\rho}\left(\bigoplus_{j=1}^{m_{i}} \bigoplus_{u=1}^{l} F_{i}\right) \longrightarrow \bigoplus_{i=1}^{\rho} D\left(\operatorname{Ext}_{\Lambda}^{1}\left(C, F_{i}\right)\right) \otimes_{k} F_{i}
$$

$\varepsilon^{\prime}=\varepsilon(\mathcal{E})$ for $\mathcal{E}=\left\{\mathcal{E}_{i}^{\prime}\right\}_{1, \ldots, \rho}$, where $\varepsilon_{i}^{\prime}$, for $i=1, \ldots, \rho$, is the row matrix map $\left[\left(e_{j, u}^{(i)}\right)^{*} \otimes\right.$ $-]_{j, u}: \bigoplus_{j=1}^{m_{i}} \bigoplus_{u=1}^{l} F_{i} \longrightarrow D\left(\operatorname{Ext}_{\Lambda}^{1}\left(C, F_{i}\right)\right) \otimes_{k} F_{i}$ whose $(j, u)$ th component is given by the mapping $f_{j, u} \mapsto\left(e_{j, u}^{(i)}\right)^{*} \otimes f_{j, u}$, for $f_{j, u} \in F_{i}$. Now we can formulate the second claim.

$2^{o} \quad \ddot{\varphi} \circ \varepsilon=\varepsilon^{\prime} \circ \pi$.

Observe that

$$
\left[\eta_{C}^{\overline{\mathcal{F}}}\right]=\varepsilon \cdot e=\operatorname{Ext}^{1}(C, \varepsilon)(e) \in \operatorname{Ext}_{A \otimes \Lambda}^{1}\left(C, \bigoplus_{i=1}^{\rho} D^{A}\left(\operatorname{Ext}_{A \otimes \Lambda}^{1}\left(C, \bar{F}_{i}\right)\right) \otimes_{A} \bar{F}_{i}\right)
$$

where $e=\left(e_{j}^{(i)}\right)_{i, j} \in \operatorname{Ext}_{A \otimes \Lambda}^{1}\left(C, \bigoplus_{i=1}^{\rho} \bigoplus_{j=1}^{m_{i}} \bar{F}_{i}\right)\left(=\bigoplus_{i=1}^{\rho} \bigoplus_{j=1}^{m_{i}} \operatorname{Ext}_{A \otimes \Lambda}^{1}\left(C, \bar{F}_{i}\right)\right)$, that

$$
\left[\eta_{C}^{\mathcal{F}}\right]=\varepsilon^{\prime} \cdot e^{\prime}=\operatorname{Ext}^{1}\left(C, \varepsilon^{\prime}\right)\left(e^{\prime}\right) \in \operatorname{Ext}_{\Lambda}^{1}\left(C, \bigoplus_{i=1}^{\rho} D\left(\operatorname{Ext}_{\Lambda}^{1}\left(C, F_{i}\right)\right) \otimes_{k} F_{i}\right)
$$

where $\quad e^{\prime}=\left(e_{j, u}^{(i)}\right)_{i, j, u} \in \operatorname{Ext}_{\Lambda}^{1}\left(C, \bigoplus_{i=1}^{\rho} \bigoplus_{j=1}^{m_{i}} \bigoplus_{u=1}^{l} F_{i}\right) \quad\left(=\bigoplus_{i=1}^{\rho} \bigoplus_{j=1}^{m_{i}} \bigoplus_{u=1}^{l} \operatorname{Ext}_{\Lambda}^{1}\right.$ $\left(C, F_{i}\right)$ ), by Theorem 4.1 , and that $e^{\prime}=\pi \cdot e=\operatorname{Ext}^{1}(C, \pi)(e)$. Consequently, the equality $2^{o}$ immediately implies $(*)$. 
To prove $1^{o}$ and $2^{o}$ we set $c_{u}:=\theta^{-1}\left(b_{u}^{*}\right)(\in A)$, for any $u=1, \ldots, l$. Clearly, $\mathcal{B}^{\prime}:=$ $\left\{c_{u}\right\}_{u=1, \ldots, l}$ forms a $k$-basis of $A$ and $b_{u}^{*}=\theta_{\left(c_{u}\right)}$, for every $u$. Note that for any $i=$ $1, \ldots, \rho$, we have

$$
\pi_{u}^{(i)} \cdot d=c_{u} \varphi_{i}(d)
$$

for all $d \in \operatorname{Ext}_{A \otimes \Lambda}^{1}\left(C, \bar{F}_{i}\right)$, since $\pi_{u}^{(i)} \cdot d=\widetilde{b_{u}^{*}} \cdot d=\widetilde{\theta_{\left(c_{u}\right)}} \cdot d=c_{u} \varphi_{i}(d)$ (see Proposition 4.6). Now, for a fixed $i$, applying (i) for $d=e_{j}^{(i)}$, we obtain the equalities $e_{j, u}^{(i)}=\pi_{u}^{(i)} \cdot e_{j}^{(i)}=c_{u} \varphi_{i}\left(e_{j}^{(i)}\right)$, for all $j=1, \ldots, m_{i}$ and $u=1, \ldots, l$. Consequently, $\mathcal{E}_{i}^{\prime}=$ $\left\{c_{u} \varphi_{i}\left(e_{j}^{(i)}\right)\right\}_{j=1, \ldots, m_{i} ; u=1, \ldots, l}$ and $1^{o}$ is proved.

Before we prove $2^{o}$, observe that for a fixed $i$ we have the following equalities in $D\left(\operatorname{Ext}_{\Lambda}^{1}\left(C, F_{i}\right)\right)$ :

$$
\left(e_{j, u}^{(i)}\right)^{*}=c_{u}^{*} \circ\left(e_{j}^{(i)}\right)^{A} \circ \varphi_{i}^{-1}
$$

for all $j, u$, where $c_{u}^{*} \in\left(\mathcal{B}^{\prime}\right)^{*}$, and

$$
\theta_{(a)} \circ\left(e_{j}^{(i)}\right)^{A} \circ \varphi_{i}^{-1}=\sum_{u=1}^{l} b_{u}^{*}(a)\left(e_{j, u}^{(i)}\right)^{*}
$$

for all $j$ and $a \in A$. The equality (ii) is satisfied, since by (i) $e_{j, u}^{(i)}=c_{u} \varphi_{i}\left(e_{j}^{(i)}\right)$ for all $j, u$, and hence $\left(e_{j, u}^{(i)}\right)^{*}=c_{u}^{*} \circ \varphi_{i}\left(e_{j}^{(i)}\right)^{A}=c_{u}^{*} \circ\left(e_{j}^{(i)}\right)^{A} \circ \varphi_{i}^{-1}$, where $\varphi_{i}\left(e_{j}^{(i)}\right)^{A} \in \varphi_{i}\left(\mathcal{E}_{i}\right)^{A}$ for the $A$-basis $\varphi_{i}\left(\mathcal{E}_{i}\right)$ of $\operatorname{Ext}_{\Lambda}^{1}\left(C, F_{i}\right)$. To show (iii) for a fixed $j$, note that for any $a \in A$ we have $\theta_{(a)}=\sum_{u=1}^{l} b_{u}^{*}(a) c_{u}^{*}$, since $b_{u}^{*}=c_{u} \theta_{(1)}$ and $\sum_{u=1}^{l}\left(c_{u} \theta_{(1)}\right)(a) c_{u}^{*}=$ $\sum_{u=1}^{l} \theta_{(a)}\left(c_{u}\right) c_{u}^{*}=\theta_{(a)}$. Now applying the equality above and (ii) we obtain

$$
\theta_{(a)} \circ\left(e_{j}^{(i)}\right)^{A} \circ \varphi_{i}^{-1}=\sum_{u=1}^{l} b_{u}^{*}(a)\left(c_{u}^{*} \circ\left(e_{j}^{(i)}\right)^{A} \circ \varphi_{i}^{-1}\right)=\sum_{u=1}^{l} b_{u}^{*}(a)\left(e_{j, u}^{(i)}\right)^{*}
$$

To prove $2^{o}$ it suffices to show that $\ddot{\varphi}_{i} \circ \varepsilon_{i}=\varepsilon_{i}^{\prime} \circ\left(\bigoplus_{j=1}^{m_{i}} \pi^{(i)}\right)$ for every $i$.

Fix $i$ and an element $\bar{f}=\left(a_{j} \otimes f_{j}\right)_{j} \in \bigoplus_{j=1}^{m_{i}} \bar{F}$. Then applying the definitions of all the maps we have:

$$
\left(\ddot{\varphi}_{i} \circ \varepsilon_{i}\right)(\bar{f})=\ddot{\varphi}_{i}\left(\sum_{j=1}^{m_{i}}\left(e_{j}^{(i)}\right)^{A} \otimes a_{j} \otimes f_{j}\right)=\sum_{j=1}^{m_{i}}\left(\theta_{\left(a_{j}\right)} \circ\left(e_{j}^{(i)}\right)^{A} \circ \varphi_{i}^{-1}\right) \otimes f_{j}
$$

and

$$
\begin{aligned}
\left(\varepsilon_{i}^{\prime} \circ\left(\bigoplus_{j=1}^{m_{i}} \pi^{(i)}\right)\right)(\bar{f}) & =\varepsilon_{i}^{\prime}\left(\left(b_{u}^{*}\left(a_{j}\right) f_{j}\right)_{j, u}\right)=\sum_{j=1}^{m_{i}} \sum_{u=1}^{l}\left(e_{j, u}^{(i)}\right)^{*} \otimes b_{u}^{*}\left(a_{j}\right) f_{j} \\
& =\sum_{j=1}^{m_{i}}\left(\sum_{u=1}^{l}\left(b_{u}^{*}\left(a_{j}\right) e_{j, u}^{(i)}\right)^{*}\right) \otimes f_{j} .
\end{aligned}
$$

Consequently, by (iii)

$$
\left(\ddot{\varphi}_{i} \circ \varepsilon_{i}\right)(\bar{f})=\left(\varepsilon_{i}^{\prime} \circ\left(\bigoplus_{j=1}^{m_{i}} \pi^{(i)}\right)\right)(\bar{f}) .
$$

In this way the proof of $2^{\circ}$ is complete and the theorem is proved. 


\section{8}

Let $\Lambda=k Q / I$ be a $k$-algebra of the bound quiver $(Q, I)$, where $I$ is generated by the finite set $R e l$ of relations $\varrho_{i}$ as in Section 4.2. Moreover, let $B, B^{\prime}$ be a pair of $R \otimes_{k} \Lambda$-modules from $\bmod _{R}^{\text {mat }} \Lambda$ with rank vectors $\underline{\mathrm{rk}} B=\left(m_{x}\right)_{x \in Q_{0}}$ and $\underline{\text { rk }} B^{\prime}=\left(m_{x}^{\prime}\right)_{x \in Q_{0}}$, where $R$ is a commutative $k$-algebra. Then $\operatorname{Der}\left(\Lambda, \operatorname{Hom}_{R}\left(B, B^{\prime}\right)\right)$ consists of all sequences $\delta=\left(\delta_{\alpha}\right) \in \bigoplus_{\alpha \in Q_{1}} \mathbb{M}_{m_{s(\alpha)}^{\prime} \times m_{t(\alpha)}}(R)$ satisfying the system of $R$-linear equations given by the (matrix) equalities $(*)$ from Section 4.2 , in $\mathbb{M}_{m_{s\left(Q_{i}\right)}^{\prime} \times m_{t\left(Q_{i}\right)}}(R)$, for every $i=1, \ldots,|\operatorname{Rel}|$. Similarly, $\operatorname{Der}^{0}\left(\Lambda, \operatorname{Hom}_{R}\left(B, B^{\prime}\right)\right)$ consists of all $\delta=\left(\delta_{\alpha}\right) \in \bigoplus_{\alpha \in Q_{1}} \mathbb{M}_{m_{s(\alpha)}^{\prime} \times m_{t(\alpha)}}(R)$ with the property that there exists a sequence $\chi=\left(\chi_{x}\right) \in \bigoplus_{x \in Q_{0}} \mathbb{M}_{m_{x}^{\prime} \times m_{x}}(R)$ such that the equalities $(* *)$ from Section 4.2 hold in $\mathbb{M}_{m_{s(\alpha)}^{\prime} \times m_{t(\alpha)}}(R)$, for every $\alpha \in Q_{1}$.

Fix linear ordering on the sets $Q_{0}$ and $Q_{1}$. Then setting

$$
v_{0}:=\sum_{x \in Q_{0}} m_{x}^{\prime} \cdot m_{x}, \quad v_{1}:=\sum_{\alpha \in Q_{1}} m_{s(\alpha)}^{\prime} \cdot m_{t(\alpha)}, \quad v_{2}:=\sum_{i=1}^{|R e l|} m_{s\left(\varrho_{i}\right)}^{\prime} \cdot m_{t\left(\varrho_{i}\right)}
$$

(depend only on $\underline{\mathrm{rk}} B$ and $\underline{\mathrm{rk}} B^{\prime}$ ), we have the induced bijections

$$
\begin{aligned}
& {\left[v_{0}\right] \longleftrightarrow \bigsqcup_{x \in Q_{0}}\left[m_{x}^{\prime}\right] \times\left[m_{x}\right], \quad\left[v_{1}\right] \longleftrightarrow \bigsqcup_{\alpha \in Q_{1}}\left[m_{s(\alpha)}^{\prime}\right] \times\left[m_{t(\alpha)}\right],} \\
& {\left[v_{2}\right] \longleftrightarrow \bigsqcup_{i=1}^{|\operatorname{Rel}|}\left[m_{s\left(\varrho_{i}\right)}^{\prime}\right] \times\left[m_{t\left(Q_{i}\right)}\right]}
\end{aligned}
$$

where the induced linear orderings in all the sets $\left[\mathrm{m}^{\prime}\right] \times[\mathrm{m}]$ are given by the sequence $(1,1), \ldots,(1, m),(2,1), \ldots,\left(m^{\prime}, m\right)$, and the associated isomorphisms

$$
R^{v_{0}} \cong \bigoplus_{x \in Q_{0}} \mathbb{M}_{m_{x}^{\prime} \times m_{x}}(R), \quad R^{v_{1}} \cong \bigoplus_{\alpha \in Q_{1}} \mathbb{M}_{m_{s(\alpha)}^{\prime} \times m_{t(\alpha)}}(R), \quad R^{v_{2}} \cong \bigoplus_{i=1}^{|R e l|} \mathbb{M}_{m_{s\left(e_{i}\right)}^{\prime} \times m_{t\left(Q_{i}\right)}}(R)
$$

Lemma There exist matrices $\mathcal{M}:=\mathcal{M}\left(B, B^{\prime}\right) \in \mathbb{M}_{v_{2} \times v_{1}}(R), \mathcal{M}^{0}:=\mathcal{M}^{0}\left(B, B^{\prime}\right) \in$ $\mathbb{M}_{v_{1} \times v_{0}}(R)$ canonically determined by the the pair $\left(B, B^{\prime}\right)$ such that under the identifications above

(a) $\operatorname{Der}\left(\Lambda, \operatorname{Hom}_{R}\left(B, B^{\prime}\right)\right)=\operatorname{Ker} \mathcal{M}$,

(b) $\operatorname{Der}^{0}\left(\Lambda, \operatorname{Hom}_{R}\left(B, B^{\prime}\right)\right)=\operatorname{Im} \mathcal{M}^{0} \cdot$ and $\operatorname{Hom}_{R \otimes \Lambda}\left(B, B^{\prime}\right)=\operatorname{Ker} \mathcal{M}^{0}$,

where $\mathcal{M} \cdot: R^{v_{1}} \rightarrow R^{v_{2}}, \mathcal{M}^{0} \cdot: R^{v_{0}} \rightarrow R^{v_{1}}$.

Proof The precise descriptions of $\mathcal{M}$ and $\mathcal{M}^{0}$ follow directly from the equalities (*) and $(* *)$ from Section 4.2 . 
4.9

Let $h: R \rightarrow \bar{R}$ be a commutative ring homomorphism. Then for any pair $m_{1}, m_{2} \in$ $\mathbb{N}_{m}, h$ induces a $k$-homomorphisms

$$
\hat{h}:=\hat{h}_{m_{1}, m_{2}}: \mathbb{M}_{m_{1} \times m_{2}}(R) \rightarrow \mathbb{M}_{m_{1} \times m_{2}}(\bar{R})
$$

given by $\hat{h}(\mathcal{A})=\overline{\mathcal{A}}$, where $\overline{\mathcal{A}}:=\left[\bar{a}_{i, j}\right] \in \mathbb{M}_{m_{1} \times m_{2}}(\bar{R})$, for $\mathcal{A}=\left[a_{i, j}\right] \in \mathbb{M}_{m_{1} \times m_{2}}(R)$. (We use the notation $\bar{a}:=h(a)$, for $a \in R$ ). Note that the restriction of the functor $\bar{R} \otimes_{R}-: \bmod _{R} \Lambda \rightarrow \bmod _{\bar{R}} \Lambda$ to $\bmod _{R}^{\text {mat }} \Lambda$, under the standard identifications $\bar{R} \otimes$ ${ }_{R} R^{m} \cong \bar{R}^{m}$, for $m \in \mathbb{N}$, can be regarded as a functor

$$
(\cdot): \bmod _{R}^{\mathrm{mat}} \Lambda \rightarrow \bmod _{\bar{R}}^{\mathrm{mat}} \Lambda
$$

given on objects by the mapping $B=\left(B_{\alpha}\right)_{\alpha \in Q_{1}} \mapsto \bar{B}:=\left(\bar{B}_{\alpha}\right)_{\alpha \in Q_{1}}$.

Lemma Let $B, B^{\prime}$ be a pair of $R \otimes_{k} \Lambda$-modules from $\bmod _{R} \Lambda$ and $h$ be as above. Then

(a) $\operatorname{Der}\left(\Lambda, \operatorname{Hom}_{\bar{R}}\left(\bar{B}, \bar{B}^{\prime}\right)\right)=\operatorname{Ker} \overline{\mathcal{M}}$,

(b) $\operatorname{Der}^{0}\left(\Lambda, \operatorname{Hom}_{\bar{R}}\left(\bar{B}, \bar{B}^{\prime}\right)\right)=\operatorname{Im} \overline{\mathcal{M}}^{0} \cdot$ and $\operatorname{Hom}_{\bar{R} \otimes \Lambda}\left(\bar{B}, \bar{B}^{\prime}\right)=\operatorname{Ker} \overline{\mathcal{M}}^{0}$,

where $\bar{B}=\bar{R} \otimes_{R} B, \bar{B}^{\prime}=\bar{R} \otimes_{R} B^{\prime}, \mathcal{M}:=\mathcal{M}\left(B, B^{\prime}\right), \mathcal{M}^{0}:=\mathcal{M}^{0}\left(B, B^{\prime}\right)$ and $\overline{\mathcal{M}} \cdot$ : $\bar{R}^{v_{1}} \rightarrow \bar{R}^{v_{2}}, \overline{\mathcal{M}}^{0} \cdot: \bar{R}^{v_{0}} \rightarrow \bar{R}^{v_{1}}$

Proof From the description of the functor $(\cdot)$ and the construction of the respective matrices we have $\mathcal{M}\left(\bar{B}, \bar{B}^{\prime}\right)=\overline{\mathcal{M}}$ and $\mathcal{M}^{0}\left(\bar{B}, \bar{B}^{\prime}\right)=\overline{\mathcal{M}}^{0}$. Hence, the assertions follow immediately from Lemma 4.8 .

\section{Corollary}

(a) Assume that

$$
\mathcal{U M V}=\mathcal{D}:=\left[\begin{array}{ccccc}
d_{1} & \ldots & 0 & \ldots & 0 \\
\vdots & \ddots & \vdots & & \vdots \\
0 & \ldots & d_{r} & \ldots & 0 \\
\vdots & & \vdots & & \vdots \\
0 & \ldots & 0 & \ldots & 0
\end{array}\right] \in \mathbb{M}_{v_{2} \times v_{1}}(R)
$$

for some invertible matrices $\mathcal{U} \in \mathrm{u}\left(\mathbb{M}_{v_{2} \times v_{2}}(R)\right)$ and $\mathcal{V} \in \mathrm{u}\left(\mathbb{M}_{v_{1} \times v_{1}}(R)\right)$, where $d_{1}, \ldots, d_{r} \neq 0$. Then $\operatorname{Der}\left(\Lambda, \operatorname{Hom}_{\bar{R}}\left(\bar{B}, \bar{B}^{\prime}\right)\right) \cong \bar{R}^{v_{1}-r} \oplus \operatorname{ann}_{\bar{R}} \bar{d}_{1} \oplus \cdots \oplus \operatorname{ann}_{\bar{R}} \bar{d}_{r}$ in $\bmod \bar{R}$.

(b) Assume that

$$
\mathcal{W} \mathcal{M}^{0} \mathcal{X}=\mathcal{D}^{0}:=\left[\begin{array}{ccccc}
d_{1}^{0} & \ldots & 0 & \ldots & 0 \\
\vdots & \ddots & \vdots & & \vdots \\
0 & \ldots & d_{r^{0}}^{0} & \ldots & 0 \\
\vdots & & \vdots & & \vdots \\
0 & \ldots & 0 & \ldots & 0
\end{array}\right] \in \mathbb{M}_{v_{1} \times v_{0}}(R)
$$

for some invertible matrices $\mathcal{W} \in \mathrm{u}\left(\mathbb{M}_{v_{1} \times v_{1}}(R)\right)$ and $\mathcal{X} \in \mathrm{u}\left(\mathbb{M}_{v_{0} \times v_{0}}(R)\right)$, where $d_{1}^{0}, \ldots, d_{r^{0}}^{0} \neq 0$. Then $\bar{R}^{v_{1}} / \operatorname{Der}^{0}\left(\Lambda, \operatorname{Hom}_{\bar{R}}\left(\bar{B}, \bar{B}^{\prime}\right)\right) \cong \bar{R} /\left(\bar{d}_{1}^{0}\right) \oplus \cdots \oplus \bar{R} /\left(\bar{d}_{r^{0}}^{0}\right) \oplus$ $\bar{R}^{v_{1}-r_{0}}$ in $\bmod \bar{R}$. 
Proof (a) Note that under our assumptions we have the equality $\overline{\mathcal{U}} \overline{\mathcal{M}} \overline{\mathcal{V}}=\overline{\mathcal{D}}$ in $\epsilon$ $\mathbb{M}_{v_{2} \times v_{1}}(\bar{R})$. Moreover, the matrices $\overline{\mathcal{U}} \in \mathbb{M}_{v_{2} \times v_{2}}(\bar{R})$ and $\overline{\mathcal{V}} \in \mathbb{M}_{v_{1} \times v_{1}}(\bar{R})$ are invertible. Now the assertion (a) follows immediately from Lemma 4.9(a).

The arguments in the proof of (b) are analogous.

The next result explains the role of the shape of the matrices $\mathcal{D}$ and $\mathcal{D}^{0}$ as above in case $R$ satisfies the following condition:

if $F_{1}$ is a direct summand of $F$ and $F_{1}, F$ are finitely generated free $R$-modules then so is $F / F_{1}$.

(Note that each principal ideal domain $R$ has this property).

Lemma Let $R$ be a ring satisfying $(*)$ and $\left(F_{1}^{0}, F\right),\left(F_{1}, F\right)$ two pairs of finitely generated free $R$-modules as above such that $F_{1}^{0} \subseteq F_{1}$. Then $F_{1} / F_{1}^{0}$ is also a finitely generated free $R$-module.

Proof Follows immediately, if we consider the canonical exact sequence

$$
0 \rightarrow F_{1} / F_{1}^{0} \rightarrow F / F_{1}^{0} \rightarrow F / F_{1} \rightarrow 0 .
$$

Proposition Let $R$ be a ring satisfying (*). Assume that for $B, B^{\prime}$ from $\bmod _{R}^{\text {mat }} \Lambda$, the matrices $\mathcal{M}=\mathcal{M}\left(B, B^{\prime}\right)$ and $\mathcal{M}^{0}=\mathcal{M}^{0}\left(B, B^{\prime}\right)$ satisfy simultaneously the assumptions $(a)$ and $(b)$ of Corollary 4.9. If additionally $d_{1}, \ldots, d_{r}, d_{1}^{0}, \ldots, d_{r^{0}}^{0}$ are invertible then

(E1) $\operatorname{Ext}_{R \otimes \Lambda}^{1}\left(B, B^{\prime}\right)$ is a finitely generated free $R$-module,

(E2) $\breve{h}_{B, B^{\prime}}^{\text {ext }}: \bar{R} \otimes_{R} \operatorname{Ext}_{R \otimes \Lambda}^{1}\left(B, B^{\prime}\right) \rightarrow \operatorname{Ext}_{\bar{R} \otimes \Lambda}^{1}\left(\bar{B}, \bar{B}^{\prime}\right)$ is a $\bar{R}$-isomorphism, for every ring homomorphism $h: R \rightarrow \bar{R}$, where $\bar{B}=\bar{R} \otimes_{R} B$ and $\bar{B}^{\prime}=\bar{R} \otimes_{R} B^{\prime}$ (see Section 4.3).

Proof We start by observing that $R^{v_{1}}=\bigoplus_{i=1}^{v_{1}} R \mathcal{V}^{(i)}=\bigoplus_{i=1}^{v_{1}} R\left(\mathcal{W}^{-1}\right)^{(i)}$, since $\mathcal{V}$ and $\mathcal{W}^{-1}$ are invertible, and by the assumptions, $\operatorname{Der}\left(\Lambda, \operatorname{Hom}_{R}\left(B, B^{\prime}\right)\right)=\operatorname{Ker} \mathcal{M} \cdot=$ $\bigoplus_{i=r+1}^{v_{1}} R \mathcal{V}^{(i)}$ and $\operatorname{Der}^{0}\left(\Lambda, \operatorname{Hom}_{R}\left(B, B^{\prime}\right)\right)=\operatorname{Im} \mathcal{M}^{0} \cdot=\bigoplus_{i=1}^{r^{0}} R\left(\mathcal{W}^{-1}\right)^{(i)}$ (cf. Lemma 4.8). Thus, by Lemmata 4.2 and 4.10, $\operatorname{Ext}_{R \otimes \Lambda}^{1}\left(B, B^{\prime}\right)$ is a finitely generated free $R$ module and (E1) is proved.

To prove (E2) consider the commutative diagram

$$
\begin{aligned}
& 0 \rightarrow \bar{R} \otimes_{R} \operatorname{Der}^{0}\left(\Lambda, \operatorname{Hom}_{R}\left(B, B^{\prime}\right)\right) \rightarrow \bar{R} \otimes_{R} \operatorname{Der}\left(\Lambda, \operatorname{Hom}_{R}\left(B, B^{\prime}\right)\right) \rightarrow \bar{R} \otimes_{R} \operatorname{Ext}_{R \otimes \Lambda}^{1}\left(B, B^{\prime}\right) \rightarrow 0 \\
& \downarrow_{\breve{h}^{\text {der }}{ }_{B, B^{\prime}}} \quad \downarrow_{\breve{h}^{\text {der }}}{ }_{B, B^{\prime}} \quad \downarrow^{\text {ext }}{ }_{B, B^{\prime}} \\
& 0 \rightarrow \quad \operatorname{Der}^{0}\left(\Lambda, \operatorname{Hom}_{\bar{R}}\left(\bar{B}, \bar{B}^{\prime}\right)\right) \rightarrow \operatorname{Der}\left(\Lambda, \operatorname{Hom}_{\bar{R}}\left(\bar{B}, \bar{B}^{\prime}\right)\right) \rightarrow \operatorname{Ext}_{\bar{R} \otimes \Lambda}^{1}\left(\bar{B}, \bar{B}^{\prime}\right) \rightarrow 0
\end{aligned}
$$

in $\bmod \bar{R}$, with exact rows $\bar{R} \otimes_{R} v_{B, B^{\prime}}$ and $v_{\bar{B}, \bar{B}^{\prime}}$, where

$v_{B, B^{\prime}}: \quad 0 \rightarrow \operatorname{Der}^{0}\left(\Lambda, \operatorname{Hom}_{R}\left(B, B^{\prime}\right)\right) \rightarrow \operatorname{Der}\left(\Lambda, \operatorname{Hom}_{R}\left(B, B^{\prime}\right)\right) \rightarrow \operatorname{Ext}_{R \otimes \Lambda}^{1}\left(B, B^{\prime}\right) \rightarrow 0$ 
and $v_{\bar{B}, \bar{B}^{\prime}}$ is defined in an analogous way $\left(\bar{R} \otimes_{R} v_{B, B^{\prime}}\right.$ is exact since $v_{B, B^{\prime}}$ splits by (E1), cf. also Remark 4.3). It suffices to show that $\breve{h}_{B, B^{\prime}}$ and $\breve{h}^{\text {der }}{ }_{B, B^{\prime}}$ are $\bar{R}$ isomorphisms.

Observe that $\bar{R}^{v_{1}}=\bigoplus_{i=1}^{v_{1}} R \overline{\mathcal{V}}^{(i)}=\bigoplus_{i=1}^{v_{1}} R\left(\overline{\mathcal{W}}^{-1}\right)^{(i)}, \quad \operatorname{Der}\left(\Lambda, \operatorname{Hom}_{\bar{R}}\left(\bar{B}, \bar{B}^{\prime}\right)\right)=$ $\operatorname{Ker} \overline{\mathcal{M}} \cdot=\bigoplus_{i=r+1}^{v_{1}} R \overline{\mathcal{V}}^{(i)}$ and $\operatorname{Der}^{0}\left(\Lambda, \operatorname{Hom}_{\bar{R}}\left(\bar{B}, \bar{B}^{\prime}\right)\right)=\operatorname{Im} \overline{\mathcal{M}}^{0} \cdot=\bigoplus_{i=1}^{r^{0}} R\left(\overline{\mathcal{W}}^{-1}\right)^{(i)}$ (see Lemma 4.9 and proof of Corollary 4.9.). Moreover, the standard isomorphism $\breve{h}: \bar{R} \otimes_{R} R^{v_{1}} \rightarrow \bar{R}^{v_{1}}$ sends $1 \otimes \mathcal{V}^{(i)}$ to $\overline{\mathcal{V}}^{(i)}$ and $1 \otimes\left(\mathcal{W}^{-1}\right)^{(i)}$ to $\left(\overline{\mathcal{W}}^{-1}\right)^{(i)}$. Consequently, $\breve{h}^{\text {der }}{ }_{B, B^{\prime}}$ and $\breve{h}^{\text {der }}{ }_{B, B^{\prime}}$ are the restrictions of $\breve{h}$ to the respective direct summands, so they are $R$-isomorphisms and the proof is complete.

\section{Remark}

(a) If (E1) and (E2) are satisfied for $B, B^{\prime}$ in $\bmod _{R} \Lambda$ then we have

(E3) There exists $m \in \mathbb{N}_{+}$such that $\operatorname{Ext}_{\bar{R} \otimes \Lambda}^{1}\left(\bar{B}, \bar{B}^{\prime}\right) \cong \bar{R}^{m}$, for any $R$-algebra $\bar{R}$ (i.e. ring homomorphism $h: R \rightarrow \bar{R}$ ).

(b) If $R=k[X]$, where $X$ is an affine variety, and (E3) is satisfied for $B, B^{\prime}$ in $\bmod _{R} \Lambda$, then we have

(E4) The dimension sequence $\left(\operatorname{dim}_{k} \operatorname{Ext}_{\Lambda}^{1}\left(R / m_{x} \otimes_{R} B, R / m_{x} \otimes_{R} B^{\prime}\right)\right)_{x \in X}$ is constant, where $m_{x}$ denotes the maximal ideal in $R$ corresponding to the point $x \in X$ (note that $R / m_{x} \cong k$, for any $x \in X$ ).

\subsection{1}

In fact for rings $R=k[\xi]_{f}$ all the conditions (E1 + E2), (E3) and (E4) are equivalent by the following result, which will be important in further considerations.

Proposition Let $R=k[\xi]_{f}$ be a localization of the polynomial ring $k[\xi]$, where $f \in$ $k[\xi]$, and $B, B^{\prime}$ a pair of $R \otimes_{k} \Lambda$-modules from $\bmod _{R} \Lambda$. If the dimension sequence

$$
\left(\operatorname{dim}_{k} \operatorname{Ext}_{\Lambda}^{1}\left(B(a), B^{\prime}(a)\right)\right)_{a \in k_{f}}
$$

is constant, then the conditions (E1) and (E2), for the pair $B, B^{\prime}$, are satisfied.

Proof Without loss of generality we can assume that $B, B^{\prime}$ belong to $\bmod _{R}^{\text {mat }} \Lambda$. Then it suffices only to show that the assumptions of Proposition 4.10 are satisfied.

Since $R$ is a principal ideal domain, so for $\mathcal{M}=\mathcal{M}\left(B, B^{\prime}\right)$ and $\mathcal{M}^{0}=\mathcal{M}^{0}\left(B, B^{\prime}\right)$ there exist two pairs of invertible matrices $\mathcal{U} \in \mathrm{u}\left(\mathbb{M}_{v_{2} \times v_{2}}(R)\right), \mathcal{V} \in \mathrm{u}\left(\mathbb{M}_{v_{1} \times v_{1}}(R)\right)$ and $\mathcal{W} \in \mathrm{u}\left(\mathbb{M}_{v_{1} \times v_{1}}(R)\right), \mathcal{X} \in \mathrm{u}\left(\mathbb{M}_{v_{0} \times v_{0}}(R)\right)$ such that $\mathcal{U} \mathcal{M} \mathcal{V}=\mathcal{D}$ and $\mathcal{W} \mathcal{M}^{0} \mathcal{X}=\mathcal{D}^{0}$, where $\mathcal{D} \in \mathbb{M}_{v_{2} \times v_{1}}(R)$ and $\mathcal{D}^{0} \in \mathbb{M}_{v_{1} \times v_{0}}(R)$ are diagonal matrices as in Corollary 4.9 , with $d_{1}, \ldots, d_{r}, d_{1}^{0}, \ldots, d_{r^{0}}^{0} \neq 0$ (see [20]). We prove that $d_{1}, \ldots, d_{r}, d_{1}^{0}, \ldots, d_{r^{0}}^{0} \in$ $\mathrm{u}(R)$.

Let $\partial: k_{f} \rightarrow \mathbb{N}$ be the function given by

$$
\partial(a)=\operatorname{dim}_{k} \operatorname{Ext}_{\Lambda}^{1}\left(B(a), B^{\prime}(a)\right)
$$


for $a \in k_{f}$. Note that for any $a \in k_{f}$, under the standard isomorphism $R /(\xi-a) \cong k$ induced by the projection $\pi_{a}: R \rightarrow k$, given by the mapping $\frac{g}{f^{n}} \mapsto \frac{g(a)}{f(a)^{n}}$ for $\frac{g}{f^{n}} \in R$, we have the following $k$-isomorphisms

$$
\begin{aligned}
\operatorname{Ext}_{\Lambda}^{1}\left(B(a), B^{\prime}(a)\right) & \cong \operatorname{Ext}_{\bar{R} \otimes \Lambda}^{1}\left(B(a), B^{\prime}(a)\right) \\
& \cong \operatorname{Der}\left(\Lambda, \operatorname{Hom}_{\bar{R}}\left(B(a), B^{\prime}(a)\right)\right) / \operatorname{Der}^{0}\left(\Lambda, \operatorname{Hom}_{\bar{R}}\left(B(a), B^{\prime}(a)\right)\right)
\end{aligned}
$$

where $\bar{R}=R /(\xi-a)$. Thus, $\partial(a)=\partial^{1}(a)-\partial^{0}(a)$, where $\partial^{1}(a)=\operatorname{dim}_{k} \operatorname{Der}\left(\Lambda, \operatorname{Hom}_{\bar{R}}\right.$ $\left.\left(B(a), B^{\prime}(a)\right)\right)$ and $\partial^{0}(a)=\operatorname{dim}_{k} \operatorname{Der}^{0}\left(\Lambda, \operatorname{Hom}_{\bar{R}}\left(B(a), B^{\prime}(a)\right)\right)$. By the isomorphisms from Corollary 4.9 applied to the canonical projections $\pi_{a}: R \rightarrow \bar{R} \cong k$, we obtain the equalities:

$$
\partial^{1}(a)=v_{1}-r+\epsilon^{1}(a)
$$

where $\epsilon^{1}(a):=\left|\left\{j: d_{j}(a)=0\right\}\right| \quad\left(\operatorname{ann}_{\bar{R}} \bar{d}_{j}=\bar{R} \cong k\right.$, if $\bar{d}_{j}=d_{j}(a)=0$; otherwise, $\left.\operatorname{ann}_{\bar{R}} \bar{d}_{j}=0\right)$,

$$
-\partial^{0}(a)=-r^{0}+\epsilon^{0}(a)
$$

where $\epsilon^{0}(a):=\left|\left\{j: d_{j}^{0}(a)=0\right\}\right| \quad\left(\bar{R} /\left(\bar{d}_{j}^{0}\right)=\bar{R} \cong k\right.$, if $\bar{d}_{j}^{0}=d_{j}(a)=0 ;$ otherwise, $\left.\bar{R} /\left(\bar{d}_{j}^{0}\right)=0\right)$, and hence

$$
\partial(a)=v_{1}-r-r^{0}+\epsilon^{1}(a)+\epsilon^{0}(a) .
$$

On the other hand, by assumption, $\partial: k_{f} \rightarrow \mathbb{N}$ is a constant function, so by the finiteness of the subset $Z:=\bigcup_{j=1}^{r} \mathcal{Z}_{k_{f}}\left(d_{j}\right) \cup \bigcup_{j=1}^{r^{0}} \mathcal{Z}_{k_{f}}\left(d_{j}^{0}\right) \subset k_{f}$ (all elements $d_{j}$ and $d_{j}^{0}$ are nonzero in $R$ !), we infer that $\partial(a)=v_{1}-r-r^{0}$, for every $a \in k_{f}$, and $Z=\emptyset$. Consequently, $d_{1}, \ldots, d_{r}, d_{1}^{0}, \ldots, d_{r^{0}}^{0} \in \mathrm{u}(R)$ and the proof is complete.

Now we are ready to prove Theorem 3.1.

Proof of Theorem 3.1 Fix $B$ in $\bmod _{R} \Lambda$, for $R:=k[\xi]_{f}$. Observe first that by the assumption and Proposition 4.11, the conditions (E1) and (E2) hold for all the pairs $\left(B, \tilde{F}_{i}\right), i=1, \ldots, \rho$, where $\tilde{F}_{i}:=R \otimes_{k} F_{i}$ (note that $\left.\tilde{F}_{i}(a)=R /(\xi-a) \otimes_{R} \tilde{F}_{i} \cong F_{i}\right)$. Moreover, for any commutative $k$-algebra $\bar{R}$ and algebra homomorphism $h: R \rightarrow$ $\bar{R}$, all $\bar{R}$-homomorphisms $\breve{h}^{\text {hom }} \tilde{F}_{i}, \tilde{F}_{j}$ for any $i, j=1, \ldots, \rho$, are isomorphisms, since $\breve{h}^{\text {hom }}{ }_{\tilde{F}_{i}, \tilde{F}_{j}} \circ\left(\operatorname{id}_{\bar{R}} \otimes \breve{h}_{0}^{\text {hom }}{ }_{F_{i}, F_{j}}\right)=\breve{h}_{1}^{\text {hom }}{ }_{F_{i}, F_{j}}$, where $h_{0}: k \rightarrow R$ and $h_{1}: k \rightarrow \bar{R} \otimes_{R} R$ are the canonical embeddings, and both, $\breve{h}_{0}^{\text {hom }}{ }_{F_{i}, F_{j}}$ and $\breve{h}_{1}^{\text {hom }}{ }_{F_{i}}, F_{j}$, are isomorphisms by Lemma 4.3. In particular, the category $\tilde{\mathcal{F}}$ is $R$-semisimple and the category $\overline{\mathcal{F}}=$ $\left\{\bar{F}_{1}, \ldots, \bar{F}_{\rho}\right\}$, where $\bar{F}_{i}=\bar{R} \otimes_{k} F_{i}$ for any $i=1, \ldots, \rho$, is $\bar{R}$-semisimple, since $\mathcal{F}$ is semisimple (note that $\bar{R} \otimes_{R} R \cong \bar{R}$ and $\bar{R} \otimes_{R} \tilde{F}_{i} \cong \bar{F}_{i}$ for any $i=1, \ldots, \rho$ ).

Consequently, by Theorem 4.1, the universal extension $\eta_{B}^{\widetilde{\mathcal{F}}}$ of $B$ with respect to $\widetilde{\mathcal{F}}$ exists. (Notice that the middle term $\Upsilon_{\widetilde{\mathcal{F}}}(B)$ of $\eta_{B}^{\widetilde{\mathcal{F}}}$ belongs to $\bmod _{R} \Lambda$ ). Moreover, by Theorem 4.4 , for any $\bar{R}$ and $h$ as above the universal extension $\eta_{\bar{B}}^{\overline{\mathcal{F}}}$ of $\bar{B}:=\bar{R} \otimes$ ${ }_{R} B$ with respect to $\overline{\mathcal{F}}$ exists and its middle term $\Upsilon_{\overline{\mathcal{F}}}(\bar{B})$ is described by the $\bar{R} \otimes_{k} \Lambda$ isomorphism

$$
\Upsilon_{\overline{\mathcal{F}}}(\bar{B}) \cong \bar{R} \otimes_{R} \Upsilon_{\widetilde{\mathcal{F}}}(B)
$$


Now assume that $\bar{R}=R /(\xi-a)^{l}$, for a pair $(a, l) \in k_{f} \times \mathbb{N}_{+}$, and that $h$ is a canonical projection $R \rightarrow R /(\xi-a)^{l}$. Since the algebra $A:=R /(\xi-a)^{l}$ is selfinjective and all the other assumptions of Theorem 4.6 for the $A \otimes_{k} \Lambda$-module $C:=\bar{B}=R /(\xi-$ $a)^{l} \otimes_{R} B$ are satisfied (they have been already positively verified above), we have a $\Lambda$-isomorphism

$$
\Upsilon_{\overline{\mathcal{F}}}(\bar{B})_{\Lambda} \cong \Upsilon_{\mathcal{F}}\left(\bar{B}_{\Lambda}\right)
$$

and hence, by $(*)$

$$
\Upsilon_{\widetilde{\mathcal{F}}}(B)(a, l) \cong \Upsilon_{\mathcal{F}}(B(a, l))
$$

in $\bmod \Lambda$.

As a consequence of the proof above we obtain a result which says that in good situations the universal extension construction commutes with localizations and therefore then we can simply work over $k[\xi]$ instead of over $k[\xi]_{f}$ (see also Corollary 4.12 and Remark 4.12). Note that this is definitely much more profitable also from the computational point of view (cf. Section 5.7).

Corollary Let $\Lambda, \mathcal{F}:=\left\{F_{1}, \ldots, F_{\rho}\right\}$ be as above and $\tilde{\mathcal{F}}:=\left\{\tilde{F}_{1}, \ldots, \tilde{F}_{\rho}\right\}$ be a $k[\xi]-$ category, where $\tilde{F}_{i}:=k[\xi] \otimes_{k} F_{i}$ for $i=1, \ldots, \rho$. Then $\tilde{\mathcal{F}}_{\tilde{\mathcal{F}}}$ is $k[\xi]$-semisimple and for any nonzero polynomial $f \in k[\xi]$, the $k[\xi]_{f}$-category $\widetilde{\mathcal{F}}_{f}:=\left\{\left(\tilde{F}_{1}\right)_{f}, \ldots,\left(\tilde{F}_{\rho}\right)_{f}\right\}$ is $k[\xi]_{f}$-semisimple. Moreover, if $B$ is a $k[\xi] \otimes_{k} \Lambda$-module from $\bmod _{k[\xi]} \Lambda$ such that the dimension sequence

$$
\left(\operatorname{dim}_{k} \operatorname{Ext}_{\Lambda}^{1}\left(B(a), F_{i}\right)\right)_{a \in k}
$$

is constant for every $i=1, \ldots, \rho$, then the universal extensions $\eta_{B}^{\widetilde{\mathcal{F}}}$ of $B$ with respect to $\widetilde{\mathcal{F}}$ and $\eta_{B_{f}}^{\widetilde{\mathcal{F}}_{f}}$ of $B_{f}$ with respect to $\widetilde{\mathcal{F}}_{f}$ exist,

$$
\Upsilon_{\widetilde{\mathcal{F}}_{f}}\left(B_{f}\right) \cong\left(\Upsilon_{\widetilde{\mathcal{F}}}(B)\right)_{f}
$$

in $\bmod _{k[\xi]_{f}} \Lambda$, and consequently,

$$
\left(\Upsilon_{\widetilde{\mathcal{F}}_{f}}\left(B_{f}\right)\right)(a, l) \cong\left(\Upsilon_{\widetilde{\mathcal{F}}}(B)\right)(a, l) \cong \Upsilon_{\mathcal{F}}(B(a, l))
$$

in $\bmod \Lambda$, for all $a \in k_{f}$ and $l \in \mathbb{N}_{+}$.

Proof Apply the first part of the proof and the formula $(*)$ for the case $R=k[\xi]$, $\bar{R}=k[\xi]_{f}$ and the canonical embedding $h: k[\xi] \hookrightarrow k[\xi]_{f}$.

Now we prove Theorem 3.2. We use the approach to modules over canonical algebras proposed by Geigle and Lenzing in [11]. To any canonical algebra $\Lambda=\Lambda(\mathbf{p}, \lambda)$ we associate the so-called weighted projective line $\mathbb{X}=\mathbb{X}(\mathbf{p}, \lambda)$ defined by setting $\mathbb{X}:=$ $\operatorname{Proj}^{\mathbf{L}}(S)$, where $S$ is the commutative $k$-algebra

$$
S=S(\mathbf{p}, \lambda):=k\left[X_{1}, \ldots, X_{t}\right] /\left(\left\{X_{i}^{p_{i}}-X_{1}^{p_{1}}-\lambda_{i} X_{2}^{p_{2}}\right\}_{i=3, \ldots, t}\right)
$$


admitting a natural grading $S=\bigoplus_{\vec{x} \in \mathbf{L}} S_{\vec{x}}$ by the rank one abelian ordered group $\mathbf{L}=\mathbf{L}(\mathbf{p})$ on generators $\vec{x}_{1}, \vec{x}_{2}, \ldots, \vec{x}_{t}$ with relations $p_{1} \vec{x}_{1}=p_{2} \vec{x}_{2}=\cdots=p_{t} \vec{x}_{t}=: \vec{c}$, such that the degree of each $X_{i}$ is just $\vec{x}_{i}$ and $\mathbf{L}_{+}:=\sum_{i=1}^{t} \mathbb{N} \vec{x}_{i}$. Consider the abelian hereditary category coh $\mathbb{X}$ consisting of all coherent sheaves of $\mathbf{L}$-graded modules over $\mathbb{X}$. Then the sheaf $T:=\bigoplus_{0<\vec{x}<\vec{c}} \mathcal{O}(\vec{x})$, formed by summing up the all shifted structure sheaves $\mathcal{O}(\vec{x}):=\mathcal{O}_{\mathbb{X}}(\vec{x})$ by elements in $\mathbf{L}$ such that $0 \leq \vec{x} \leq \vec{c}$, is a tilting object in coh $\mathbb{X}$ and $\operatorname{End}_{\mathbb{X}}(T) \cong \Lambda$. Moreover, the full right derived functor $\Psi:=$ $\mathbb{R} \operatorname{Hom}_{\mathbb{X}}(T,-): \mathcal{D}^{b}(\operatorname{coh} \mathbb{X}) \rightarrow \mathcal{D}^{b}(\bmod \Lambda)$, for the functor $\operatorname{Hom}_{\mathbb{X}}(T,-): \operatorname{coh} \mathbb{X} \rightarrow$ $\bmod \Lambda$, yields the triangle equivalence $\mathcal{D}^{b}(\operatorname{coh} \mathbb{X}) \simeq \mathcal{D}^{b}(\bmod \Lambda)$ (see [11] for all details).

Recall that each indecomposable element in coh $\mathbb{X}$ is a locally free sheaf, called a vector bundle, or a sheaf of finite length. If we denote by vect $\mathbb{X}$ (respectively $\operatorname{coh}_{0} \mathbb{X}$ ) the category of vector bundles (respectively finite length sheaves) on $\mathbb{X}$ and for any $\mu \in \overline{\mathbb{Q}}$, by $\mathcal{C}_{\mu}$ the full subcategory of coh $\mathbb{X}$ formed by all indecomposable sheaves $Y$ such that $\mu_{\mathbb{X}}(Y)=\mu$, where $\mu_{\mathbb{X}}(Y)$ is the slope of the sheaf $Y$, then clearly coh $\mathbb{X}=$ vect $\mathbb{X} \vee \operatorname{coh}_{0} \mathbb{X}, \operatorname{coh}_{0} \mathbb{X}=\operatorname{add} \mathcal{C}_{\infty}$ and vect $\mathbb{X}=\bigvee_{\mu \in \mathbb{Q}}\left(\operatorname{add} \mathcal{C}_{\mu}\right)$.

Let $\operatorname{coh}_{+} \mathbb{X}$ be the full subcategory of vect $\mathbb{X}$ formed by all vector bundles whose indecomposable summands $X$ satisfy the condition $\operatorname{Ext}_{\mathbb{X}}^{1}(T, X)=0$ and $\bmod _{+}(\Lambda)$ the full subcategory of $\bmod \Lambda$ formed by all $\Lambda$-modules whose indecomposable summands have positive rank. Then in particular, $\operatorname{coh}_{+} \mathbb{X}$ corresponds to $\bmod _{+}(\Lambda)$ by means of the mapping $X \mapsto \operatorname{Hom}_{\mathbb{X}}(T, X)$ under the equivalence $\Psi: \mathcal{D}^{b}(\operatorname{coh} \mathbb{X}) \stackrel{\sim}{\rightrightarrows}$ $\mathcal{D}^{b}(\bmod \Lambda)$.

Let now $\Lambda$ be a tubular canonical algebra of the type p. Then all $\mathcal{C}_{\mu}$, for $\mu \in \overline{\mathbb{Q}}$, are abelian uniserial categories, enjoying the same as $\operatorname{coh}_{0} \mathbb{X}$ tubular structure of the Auslander-Reiten quiver, given by a $\mathbb{P}^{1}(k)$-family of stable tubes of the type $\mathbf{p}$ indexed by the points of the line $\mathbb{X}$. One shows that $\mathcal{C}_{\mu} \subset \operatorname{coh}_{+} \mathbb{X}$, for every $\mu \in \mathbb{Q}_{>p}$; moreover, $\mathcal{C}_{p}^{\prime} \subset \operatorname{coh}_{+} \mathbb{X}$, where $\mathcal{C}_{p}^{\prime}$ it a full subcategory of $\mathcal{C}_{p}$ formed by all sheaves nonisomorphic to those lying on the coray ending with $\mathcal{O}(\vec{c}+\vec{\omega})$, which belongs to the stable tube of maximal rank (we denote it by $\mathcal{T}^{0}$ ), where $\vec{\omega} \in \mathbf{L}(\mathbf{p})$ is the socalled dualizing element (see $[9,11])$. Then $\mathcal{C}_{\mu}$ as above and $\mathcal{C}_{p}^{\prime}$ correspond via $\Psi$ to the subcategories $\mathcal{X}_{\mu}$, for $\mu>p$, and $\mathcal{X}_{p}$ of $\bmod _{+} \Lambda$ respectively. Note that this correspondence preserves tubes with the one exception, where "the intersection" $\mathcal{T}^{0} \cap \mathcal{C}_{p}^{\prime}$ corresponds to the unique nonstable tube in $\mathcal{X}_{p}$.

Proof of Theorem 3.2 Let $\mu \in \mathbb{Q}$ be a fixed rational number and $\mathcal{F}=\mathcal{F}(\mathcal{T})$ the semisimple $k$-category formed by all quasi-simple objects in a fixed (stable) tube $\mathcal{T}$ in $\mathcal{C}_{\mu} \subset$ coh $\mathbb{X}$ of the rank $\rho=\rho_{\mathcal{T}}$, where $\mathbb{X}$ is the weighted projective line associated to $\Lambda$. By [15] there exists a pair of mutually inverse equivalences

$$
\mathcal{D}^{b}(\operatorname{coh} \mathbb{X}) \underset{\mathcal{R}}{\stackrel{\mathcal{L}}{\rightleftarrows}} \mathcal{D}^{b}(\operatorname{coh} \mathbb{X})
$$

called tubular mutations with respect to $\mathcal{F}$. They have various nice properties, in particular preserve tubes. Moreover, for an indecomposable $X$ in coh $\mathbb{X}$ we have

$$
\mathcal{R}(X) \cong \Upsilon_{\mathcal{F}}(X)
$$

provided $\mu(X)>\mu$ (a slight generalization of [16, Corollary 5.2.3]). Observe that this formula allows to determine easily the slope $\mu(\mathcal{R}(X))$ for any indecomposable object $X$ in $\mathcal{C}_{\mu^{\prime}}$, for a fixed $\mu^{\prime}>\mu$. 
To compute precisely $\mu(\mathcal{R}(X))$, for a fixed $X$ in $\mathcal{C}_{\mu^{\prime}}$ with quasi-length $l:=\ell(X)$ and $\tau$-period $\rho^{\prime}:=\rho(X)$, we use the Riemann-Roch formula for the pair $\left(X, F_{1}\right)$. Since $\mu<\mu^{\prime}$ implies $\operatorname{Hom}\left(X, F_{i}\right)=0$, it has the form

$$
-\sum_{j=1}^{p} \operatorname{dim}_{k} \operatorname{Ext}^{1}\left(X, \tau^{j} F_{1}\right)=\left|\begin{array}{cc}
\operatorname{rk} X & \operatorname{rk} F_{1} \\
\operatorname{deg} X & \operatorname{deg} F_{1}
\end{array}\right|
$$

(see [11]), and this immediately yields the equality

$$
\sum_{i=1}^{\rho} \operatorname{dim}_{k} \operatorname{Ext}^{1}\left(X, F_{i}\right)=\operatorname{lm}\left(r d^{\prime}-r^{\prime} d\right)
$$

where $d^{\prime}:=\frac{\operatorname{deg} X}{l m^{\prime}}, r^{\prime}:=\frac{\mathrm{rk} X}{l m^{\prime}}, d:=\frac{\operatorname{deg} F_{1}}{m}, r:=\frac{\mathrm{rk} F_{1}}{m}, m:=\frac{p}{\rho}, m^{\prime}:=\frac{p}{\rho^{\prime}}(\in \mathbb{N})$. (It is well known that then $\mu^{\prime}=\mu(X)=\frac{d^{\prime}}{r^{\prime}}$ and $\mu=\mu\left(F_{1}\right)=\frac{d}{r}$ are irreducible fraction presentations. Applying the additive functions deg and rk to the exact sequence $\eta_{B}^{\mathcal{F}}$ (see Section 4.1), we obtain by $(*)$ the equalities

$$
\operatorname{deg} \mathcal{R}(X)=l m^{\prime}\left(r d^{\prime}-r^{\prime} d\right) \operatorname{deg} F_{1}+\operatorname{deg} X
$$

and

$$
\operatorname{rk} \mathcal{R}(X)=l m^{\prime}\left(r d^{\prime}-r^{\prime} d\right) \operatorname{rk} F_{1}+\operatorname{rk} X
$$

(deg and rk are $\tau$-invariant!). Consequently, we have

$$
\mu(\mathcal{R}(X))=\frac{\operatorname{deg} \mathcal{R}(X)}{\operatorname{rk} \mathcal{R}(X)}=\frac{\operatorname{lm}\left(r d^{\prime}-r^{\prime} d\right) m d+l m^{\prime} d^{\prime}}{l m^{\prime}\left(r d^{\prime}-r^{\prime} d\right) m r+l m^{\prime} r^{\prime}}=\frac{d m\left(r d^{\prime}-r^{\prime} d\right)+d^{\prime}}{r m\left(r d^{\prime}-r^{\prime} d\right)+r^{\prime}}\left(=: \mu^{\prime \prime}\right)
$$

(so in fact $\mu(\mathcal{R}(X))=\mu^{\prime \prime}\left(\mu, \mu^{\prime}, \rho_{\mathcal{T}}\right)$ !).

In particular, we also obtain that for any $\mu^{\prime}>\mu, \mathcal{R}$ induces a functor

$$
\mathcal{R}_{\mid}: \mathcal{C}_{\mu^{\prime}} \longrightarrow \mathcal{C}_{\mu^{\prime \prime}}
$$

where $\mu^{\prime \prime}=\mu^{\prime \prime}\left(\mu, \mu^{\prime}, \rho_{\mathcal{T}}\right)$ is as above.

Assume that $p \leq \mu<\mu^{\prime}$ (hence $d, r, d^{\prime}, r^{\prime} \in \mathbb{Z}_{\geq 1}$ ) and $\mathcal{T} \neq \mathcal{T}^{0}$, if $\mu=p$. Then we have $\mu^{\prime \prime}>p$, since

$$
p\left(r m\left(r d^{\prime}-r^{\prime} d\right)+r^{\prime}\right)<\mu r m\left(r d^{\prime}-r^{\prime} d\right)+\mu^{\prime} r^{\prime}=d m\left(r d^{\prime}-r^{\prime} d\right)+d^{\prime}
$$

Thus, $\mathcal{F}, \mathcal{C}_{\mu^{\prime}}$ and $\mathcal{C}_{\mu^{\prime \prime}}$ are contained in $\operatorname{coh}_{+} \mathbb{X}$ so they can be regarded as subcategories of $\bmod _{+} \Lambda$ and identified with $\Psi(\mathcal{F}), \mathcal{X}_{\mu^{\prime}}$ and $\mathcal{X}_{\mu^{\prime \prime}}$, respectively. Moreover, observe that then via $\Psi_{\mid \operatorname{coh}_{+} \mathbb{X}}=\operatorname{Hom}(T,-)_{\mid \operatorname{coh}_{+} \mathbb{X}}$ we have also $\eta_{\mathcal{F}}^{X}=\eta_{\Psi(\mathcal{F})}^{\Psi(X)}, \Omega_{\mathcal{F}}(B)=$ $\Omega_{\Psi(\mathcal{F})}(\Psi(X))$ and $\Upsilon_{\mathcal{F}}(X)=\Upsilon_{\Psi(\mathcal{F})}(\Psi(X))$. From now on we will freely use all these identifications.

Now we complete the proof of the theorem. Let now $B$ and $\mathcal{F}$ be as in the statement. (Note that we are exactly in the situation discussed above.) Then the dimension sequence

$$
\left(\operatorname{dim}_{k} \operatorname{Ext}_{\Lambda}^{1}\left(B(a), F_{i}\right)\right)_{a \in k_{f}}
$$

is constant for every $i=1, \ldots, \rho$, since $[B(a)]=[X]$ in $\mathrm{K}_{0}(\mathbb{X})$, for all $a \in k_{f}$, where $X$ is a quasi-simple object in a fixed homogeneous tube in $\mathcal{C}_{\mu^{\prime}}$, and hence,

$$
\operatorname{dim}_{k} \operatorname{Ext}_{\Lambda}^{1}\left(B(a), F_{i}\right)=-\operatorname{eulf}\left([X],\left[F_{i}\right]\right)
$$


where eulf $: \mathrm{K}_{0}(\mathbb{X})^{2} \rightarrow \mathbb{Z}$ is the Euler form. Then by Theorem 3.1, the universal extension $\eta_{B}^{\widetilde{\mathcal{F}}}$ and $k[\xi]_{f} \otimes_{k} \Lambda$-module $\Upsilon_{\widetilde{\mathcal{F}}}(B)$ in $\bmod _{k[\xi]_{f}} \Lambda$ are well defined. Moreover, for any pair $(a, l) \in k[\xi]_{f} \times \mathbb{N}_{+}$we have $\Lambda$-isomorphisms

$$
\Upsilon_{\widetilde{\mathcal{F}}}(B)(a, l) \cong \Upsilon_{\mathcal{F}}(B(a, l))
$$

On the other hand, from $[15,16], \mathcal{R}_{\mid}: \mathcal{X}_{\mu^{\prime}}^{0} \rightarrow \mathcal{X}_{\mu^{\prime \prime}}^{0}$ is an equivalence, where $\mathcal{X}_{\mu^{\prime}}^{0}$ and $\mathcal{X}_{\mu^{\prime \prime}}^{0}$ denote the subcategories of all homogeneous modules in $\mathcal{X}_{\mu^{\prime}}$ and $\mathcal{X}_{\mu^{\prime}}$ respectively, so by our assumption on $B$, the functor

$$
\mathcal{R}_{\mid} \circ\left(-\otimes_{k[\xi]_{f}} B\right): \bmod k[\xi]_{f} \longrightarrow \operatorname{add} \mathcal{X}_{\mu^{\prime \prime}}^{0}
$$

yields a bijection between the sets of isoclasses of indecomposable objects in $\bmod k[\xi]_{f}$ and $\mathcal{X}_{\mu^{\prime \prime}}^{0}$. Consequently, by $(* *)$, so does the functor

$$
-\otimes_{k[\xi]_{f}} \Upsilon_{\tilde{\mathcal{F}}}(B): \bmod k[\xi]_{f} \longrightarrow \operatorname{add} \mathcal{X}_{\mu^{\prime \prime}}^{0}
$$

and in this way the proof is complete.

It turns out that if the bimodule $B$ parametrizing all homogeneous indecomposable modules in the slope $\mu^{\prime}$ is a localization of the bimodule from $\bmod _{k[\xi]} \Lambda$ then the same holds true for the universal extension $\Upsilon_{\widetilde{\mathcal{F}}}(B)$ parametrizing all homogeneous indecomposable modules in the slope $\mu^{\prime \prime}$. More precisely, we have the following fact.

Corollary Let $\Lambda, B, \mu^{\prime}, \mathcal{F}, \widetilde{\mathcal{F}}$ and $\Upsilon_{\widetilde{\mathcal{F}}}(B)$, for a fixed $p \leq \mu<\mu^{\prime}$ and tube $\mathcal{T}$ in $\mathcal{X}_{\mu}$ be as in Theorem 3.2. If additionally $B \cong \dot{B}_{f}$ for some $\dot{B}$ in $\bmod _{k[\xi]} \Lambda$ such that $\dot{B}(a)$ belongs to $\mathcal{X}_{\mu^{\prime}}$ also for all $a \in \mathcal{Z}(f)$, then $\Upsilon_{\dot{\mathcal{F}}}(\dot{B})$ for the $k[\xi]$-semisimple category $\dot{\mathcal{F}}:=\left\{k[\xi] \otimes_{k} F_{1}, \ldots, k[\xi] \otimes_{k} F_{\rho}\right\}$ exits,

$$
\Upsilon_{\mathcal{F}}(\dot{B})_{f} \cong \Upsilon_{\widetilde{\mathcal{F}}}(B)
$$

and $\Upsilon_{\dot{\mathcal{F}}}(\dot{B})(a)$ belongs to $\mathcal{X}_{\mu^{\prime \prime}}$ for all $a \in \mathcal{Z}(f)$. Moreover, $\Upsilon_{\dot{\mathcal{F}}}(\dot{B})$ induces a functor $-\otimes_{k[\xi]} \Upsilon_{\mathcal{F}}(\dot{B}): \bmod k[\xi] \rightarrow$ add $\mathcal{X}_{\mu^{\prime \prime}}$, which yields an injection between the respective sets of isoclasses of indecomposable objects, provided so does $-\otimes_{k[\xi]} \dot{B}: \bmod k[\xi] \rightarrow$ add $\mathcal{X}_{\mu^{\prime}}$

Proof We show similarly as above that $\left(\operatorname{dim}_{k} \operatorname{Ext}_{\Lambda}^{1}\left(\dot{B}(a), F_{i}\right)\right)_{a \in k}$ is a constant sequence for every $i$, and then by Theorem 3.1, the universal extension $\eta_{\dot{B}}^{\dot{\mathcal{F}}}$ exists. Now applying Corollary 4.11, we obtain the required isomorphism. The remaining assertions follow by arguments analogous to those used in the previous proof.

\section{Remark}

(a) For any $d \in \mathbb{Z}_{\geq p}$, the matrix bimodule $\dot{B}:=B^{(d)}$ has the property that $\dot{B}(a)$ belongs to $\mathcal{X}_{\mu^{\prime}}$ for all $a \in \mathcal{Z}(f)$, where $B^{(d)}$ from $\bmod _{k[\xi]}^{\operatorname{mat}} \Lambda$ is as in [9, Table 4.7] and $\mu^{\prime}=d$. Clearly, due to [9, Theorem 4.1], $B:=(\dot{B})_{f}$ satisfies the assumptions of Theorem 3.2. Moreover, the functor $-\otimes_{k[\xi]} \dot{B}: \bmod k[\xi] \rightarrow$ add $\mathcal{X}_{d}$ yields an injection between the respective sets of isoclasses of indecomposable objects. All these properties follow by the very construction from the fact that they hold for $d=p($ see [9]). 
(b) Roughly speaking the corollary says that if we start with an integral slope and apply inductively our construction to create the bimodules $B^{(v)}$ (from $\bmod _{k[\xi]_{f}} \Lambda$ ) parametrizing $\mathcal{X}_{v}^{0}$, for the consecutive slopes $v$, then we can compute universal extensions $\dot{B}^{(v)}$ for modules over $k[\xi] \otimes_{k} \Lambda$ (instead of over $\left.k[\xi]_{f} \otimes_{k} \Lambda\right)$ and next to localize them to $k[\xi]_{f}$. Moreover, we have even that all the functors $-\otimes_{k[\xi]}\left(\dot{B}^{(v)}\right): \bmod k[\xi] \rightarrow$ add $\mathcal{X}_{v}$ yield injections between the respective sets of isoclasses of indecomposable objects.

\section{The Proof of the Main Result}

5.1

We start by some results which yield a precise control of the "slope changes" given by the formula $(*)$ in Section 3.2 and allow to reduce canonically the rational slopes from $\mathbb{Q}_{\geq p}$ to integers in $\mathbb{Z}_{\geq p}$, for a fixed $p \in \mathbb{N}_{+}$.

We set $\overline{\mathbb{Q}}:=\mathbb{Q} \cup\{\infty\}$. We will naturally identify $\overline{\mathbb{Q}}$ with the quotient set $\mathbb{Z}_{0}^{2} / \sim$, where $\mathbb{Z}_{0}^{2}:=\mathbb{Z}^{2} \backslash\{(0,0)\}$ and $\sim \subset \mathbb{Z}_{0}^{2} \times \mathbb{Z}_{0}^{2}$ is the proportionality relation.

\section{Lemma}

(a) For any $m \in \mathbb{N}_{+}$and $\mu=\frac{d}{r} \in \overline{\mathbb{Q}}$, where $\operatorname{gcd}(r, d)=1$, the function $\varsigma: \overline{\mathbb{Q}} \rightarrow \overline{\mathbb{Q}}$ defined by the mapping $\mu^{\prime} \mapsto \mu^{\prime \prime}\left(\mu, \mu^{\prime} ; m\right):=\frac{d m(r x-d y)+x}{r m(r x-d y)+y}$, for $\mu^{\prime}=\frac{x}{y} \in \overline{\mathbb{Q}}$, is a bijection. The unique $\mu^{\prime} \in \overline{\mathbb{Q}}$ such that $\varsigma\left(\mu^{\prime}\right)=\mu^{\prime \prime}$, for $\mu^{\prime \prime}=\frac{a}{b} \in \overline{\mathbb{Q}}$, has the form $\mu^{\prime}=\frac{x}{y}$, where $x=a+d^{2} m b-d m r a$ and $y=b-r^{2} m a+d m r b$.

(b) If $r=1$ then for any $\mu^{\prime \prime}=\frac{a}{b} \in \mathbb{Q} \backslash\left\{d+\frac{1}{m}\right\}$, with $b \in \mathbb{Z}_{\geq 1}$, we have $\mu<\mu^{\prime}$ if and only if $d<\mu^{\prime \prime}<d+\frac{1}{m}$, or equivalently $\mu=\left\lfloor\mu^{\prime \prime}\right\rfloor\left(=\mathrm{quo}_{b}(a)=d\right)$ and $0<\mu^{\prime \prime}-\left\lfloor\mu^{\prime \prime}\right\rfloor<\frac{1}{m}\left(\mu^{\prime}=\infty\right.$, if $\left.\mu^{\prime \prime}=d+\frac{1}{m}\right)$.

Proof (a) We start by noticing that for any matrix $M=\left[\begin{array}{ll}A & B \\ C & D\end{array}\right] \in \mathbb{M}_{2}(\mathbb{Z})$ with $\operatorname{det} M \neq 0$, the multiplication $M \cdot: \mathbb{Z}^{2} \rightarrow \mathbb{Z}^{2}$ induces a bijection $\varsigma=\varsigma_{M}: \overline{\mathbb{Q}} \rightarrow \overline{\mathbb{Q}}$, given by the formula $\varsigma\left(\mu^{\prime}\right)=\frac{A x+B y}{C x+D y}$, where $\mu^{\prime}=\frac{x}{y}$ is a fraction presentation of $\mu^{\prime} \in \overline{\mathbb{Q}}$. Clearly, if $M^{\prime}$ is another such matrix then we have $\varsigma_{M^{\prime}} \circ \varsigma_{M}=\varsigma_{M^{\prime}} M$. It is easily seen that the mapping $M \mapsto \varsigma_{M}$ yields a group homomorphism $\varsigma_{(-)}: \mathrm{Sl}_{2}(\mathbb{Z}) \rightarrow$ $\mathcal{S}(\overline{\mathbb{Q}})$, with $\operatorname{Ker} \varsigma_{(-)}=\left\{ \pm I_{2}\right\}$, where $\mathcal{S}(\overline{\mathbb{Q}})$ is the group of all bijections of the set $\overline{\mathbb{Q}}$. Hence, $\varsigma_{M}^{-1}=\varsigma_{M^{-1}}$, for any $M \in \mathrm{Sl}_{2}(\mathbb{Z})$, and the unique $\mu^{\prime} \in \overline{\mathbb{Q}}$ such that $\varsigma_{M}\left(\mu^{\prime}\right)=$ $\mu^{\prime \prime}$, for an arbitrary fraction presentation $\mu^{\prime \prime}=\frac{a}{b}$ of $\mu^{\prime \prime} \in \overline{\mathbb{Q}}$, is given by the formula $\mu^{\prime}=\frac{x}{y}$, where $x=D a-B b$ and $y=-C a+A b$.

Now observe that the map $\varsigma: \overline{\mathbb{Q}} \rightarrow \overline{\mathbb{Q}}$, defined by $\mu^{\prime} \mapsto \mu^{\prime \prime}\left(\mu, \mu^{\prime} ; m\right)$, has the form $\varsigma=\varsigma_{M}$ for the matrix $M:=\left[\begin{array}{cc}1+d m r & -d^{2} m \\ r^{2} m & 1-d m r\end{array}\right]$, and that det $M=1$. Consequently, the assertion follows immediately from the formulas for $x$ and $y$ given above.

(b) Follows easily by an analysis of the elementary inequality $\frac{a+d^{2} m b-d m a}{b-m a+d m b}>d$ dependently on the sign of denominator. 
Corollary If under the notation above $m, r=1$, then for $\mu^{\prime \prime}=\frac{a}{b} \in \mathbb{Q}_{\geq 0} \backslash \mathbb{Z}$, with $b \in$ $\mathbb{Z}_{\geq 1}$, we have

$$
\mu^{\prime}>\mu \Leftrightarrow \mu=\left\lfloor\mu^{\prime \prime}\right\rfloor\left(=\operatorname{quo}_{b}(a)=d\right),
$$

and then $\mu^{\prime}=\frac{a-\mathrm{quo}_{b}(a) \operatorname{rem}_{b}(a)}{b-\operatorname{rem}_{b}(a)}=\mathrm{quo}_{b}(a)+\frac{\operatorname{rem}_{b}(a)}{b-\operatorname{rem}_{b}(a)}\left(0<\operatorname{rem}_{b}(a) !\right) ;$ in particular, if $\mu^{\prime \prime} \in \mathbb{Q}_{\geq p}$, for a fixed $p \in \mathbb{N}$, then $\mu, \mu^{\prime} \in \mathbb{Q}_{\geq p}$, and $\mu<\mu^{\prime \prime}<\mu^{\prime}$.

Proof The first assertion follows immediately from (b), the formula for $\mu^{\prime}$ from the equalities $\mu^{\prime}=\frac{a+d^{2} b-d a}{b-a+d b}$ and $d=\mathrm{quo}_{b}(a)$. The remaining assertions are now straightforward.

\section{Remark}

(a) $b-\operatorname{rem}_{b}(a)<b$, since $\mu^{\prime \prime} \notin \mathbb{Z}$.

(b) $\operatorname{gcd}\left(a-l \mathrm{quo}_{b}(a) \operatorname{rem}_{b}(a), b-l \operatorname{rem}_{b}(a)\right)=\operatorname{gcd}\left(b-l \operatorname{rem}_{b}(a), \operatorname{rem}_{b}(a)\right)=1$, for any $l \in \mathbb{N}$, if $\operatorname{gcd}(a, b)=1$.

Further on we set for simplicity $\mu^{\prime \prime}\left(\mu, \mu^{\prime}\right):=\mu^{\prime \prime}\left(\mu, \mu^{\prime} ; 1\right)$.

Proposition Let $p \in \mathbb{N}$ be a fixed number. For any $\mu^{\prime \prime} \in \mathbb{Q} \geq p$ there exist uniquely determined $s=s\left(\mu^{\prime \prime}\right) \in \mathbb{N}$ and the pair $\mu^{\prime \prime}:=\left(\left(\mu_{i}\right)_{i=1, \ldots, s},\left(\mu_{i}^{\prime}\right)_{i=0, \ldots, s}\right)$ of sequences satisfying the following conditions:

- $s \geq 1$ if and only if $\mu^{\prime \prime} \notin \mathbb{Z}$,

- $\mu_{0}^{\prime}=\mu^{\prime \prime}$,

- $\mu_{1}, \ldots, \mu_{s} \in \mathbb{Z}_{\geq p}, \mu_{1}^{\prime}, \ldots, \mu_{s-1}^{\prime} \in \mathbb{Q}_{\geq p} \backslash \mathbb{Z}$ and $\mu_{s}^{\prime} \in \mathbb{Z}_{\geq p}$,

- $\mu_{i}<\mu_{i}^{\prime}$, for every $i \geq 1$,

- $\mu_{i-1}^{\prime}=\mu^{\prime \prime}\left(\mu_{i}, \mu_{i}^{\prime}\right)$, for every $i \geq 1$.

For any pair $\mu^{\prime \prime}$ as above we always have inequalities $\mu_{0}^{\prime}<\mu_{1}^{\prime}<\cdots<\mu_{s}^{\prime}$.

Proof Note that the uniqueness property is clear from the Corollary 5.1. We show the existence applying the induction on $b \in \mathbb{N}_{+}$, where $\mu^{\prime \prime}=\frac{a}{b}$ is such that $\operatorname{gcd}(a, b)=1$. If $b=1$ then $\mu^{\prime \prime}$ is an integer and we set $s:=0$ and $\mu_{0}^{\prime}=\mu^{\prime \prime}$ (the sequence is empty). If $b \geq 2$ then $\mu^{\prime \prime} \in \mathbb{Q}_{\geq p} \backslash \mathbb{Z}$. Now the assertion follows from the Corollary 5.1 and the inductive assumption, since we have the inequality $b-$ $\operatorname{rem}_{b}(a)<b$.

\section{Remark}

(a) The sequence $\mu^{\prime \prime}$ can be algorithmically determined from $\mu^{\prime \prime}$.

(b) If we know $\mu_{s}^{\prime}$ and the sequence $\mu_{s}, \ldots, \mu_{1}$ then we can easily reconstruct the values $\mu_{s-1}^{\prime}, \ldots, \mu_{0}^{\prime}=\mu^{\prime \prime}$.

(c) If $\left(\mu^{\prime \prime}\right)^{\star}=\mu_{i}^{\prime}$, for some $i$, then $s^{\star}=s-i$, where $s^{\star}:=s\left(\left(\mu^{\prime \prime}\right)^{\star}\right)$, and the pair $\left(\mu^{\prime \prime}\right)^{\star}=\left(\left(\mu_{i^{\star}}^{\star}\right)_{i^{\star}=1, \ldots, s^{\star}},\left(\left(\mu^{\prime}\right)_{i^{\star}}^{\star}\right)_{i^{\star}=0, \ldots, s^{\star}}\right)$ of sequences defined by $\left(\mu^{\prime \prime}\right)^{\star}$ is given by the formulas $\mu_{i^{\star}}^{\star}=\mu_{i^{\star}+i}$ and $\left(\mu^{\prime}\right)_{i^{\star}}^{\star}=\mu_{i^{\star}+i}^{\prime}$. 
Theorem Let $\Lambda$ be a canonical tubular algebra of the type $\mathbf{p}=\left(p_{1}, \ldots, p_{t}\right)$ and $f=$ $f_{\mathbf{p}}$. Then for any $q \in \mathbb{Q}_{\geq p}$, if $\mathbf{p}=(2,2,2,2),(3,3,3),(2,4,4)$; respectively, $q \in\{6\} \cup$ $\mathbb{Q} \geq 7$, if $\mathbf{p}=(2,3,6)$, the $k[\xi] \otimes_{k} \Lambda$-module $B^{(q)}$ defined by the formula

$$
B^{(q)}:=\left(\Upsilon_{\mu_{1}} \circ \cdots \circ \Upsilon_{\mu_{s}}\right)\left(B^{\left(\mu_{s}^{\prime}\right)}\right)
$$

is well defined, belongs to $\bmod _{k[\xi]} \Lambda$ and its localization $\left(B^{(q)}\right)_{f}$ yields a parametrizing bimodule for all indecomposable $\Lambda$-modules from the family of all homogeneous tubes in $\mathcal{X}_{q}$, where $\mu_{s}^{\prime}$ and $\mu_{1}, \ldots, \mu_{s}$ are elements of the sequences forming $\mu^{\prime \prime}$, for $\mu^{\prime \prime}:=q$.

Both, the formulation of the theorem and its proof, heavily depend on Lemma 3.3, formulated without the proof. Therefore we start by proving this important result.

Proof of Lemma 3.3 Fix p. Note first that for any $d \geq 2 p$ (resp. $d>2 p$, if $\mathbf{p}=$ $(2,3,6))$ and the sequence $\left(u ;\left(u_{i}\right)\right) \in \Theta^{(d)}$, the module $M^{\left(u-1 ;\left(u_{i}\right)\right)}$ is well defined, since $\left(u-1 ;\left(u_{i}\right)\right) \in \Theta^{(d-p)}$, and clearly

$$
\underline{\operatorname{dim}} M^{\left(u ;\left(u_{i}\right)\right)}=11+\underline{\operatorname{dim}} M^{\left(u-1 ;\left(u_{i}\right)\right)}
$$

where $11 \in \mathbb{N} Q_{0}$ is the vector with all coordinates equal to 1 . Next applying the definition of the degree function, we check case by case that $\operatorname{deg} M^{\left(u ;\left(u_{i}\right)\right)}=d$ for every $\left(u ;\left(u_{i}\right)\right) \in \Theta^{(d)}$, with $d=p, \ldots, 2 p-1$ (resp. $d=p+1, \ldots, 2 p$, if $\mathbf{p}=$ $(2,3,6))$. Now, since deg $11=p$, we infer from $(*)$ by induction arguments that the equality $\operatorname{deg} M^{\left(u ;\left(u_{i}\right)\right)}=d$ holds for all $d \geq p$ (resp. $\left.d>p\right)$. Consequently, each $\mathcal{F}^{(d)}$ consists of $p$ nonisomorphic quasi-simple objects in $\mathcal{X}_{d}$, since rk $F=1$ for every $F \in \operatorname{ob} \mathcal{F}^{(d)}$.

To prove that $\mathcal{F}^{(d)}$, for the respective $d$, has the form $\mathcal{F}^{(d)}=\mathcal{F}(\mathcal{T})$, where $\mathcal{T}$ is a tube in $\mathcal{X}_{d}$ of maximal rank $\rho_{\mathcal{T}}=p$, it suffices to show that for every $F \in$ ob $\mathcal{F}^{(d)}$, we have $\phi_{\Lambda}(\underline{\operatorname{dim}} F)=\underline{\operatorname{dim}} F^{\prime} \neq \underline{\operatorname{dim}} F$, for some $F^{\prime} \in \operatorname{ob} \mathcal{F}^{(d)}$, where $\phi_{\Lambda}$ is the Coxeter transformation for $\Lambda$ (see [2, III.3] for the definition). Note that this implies an isomorphism $\tau_{\Lambda}(F) \cong F^{\prime}$, since proj.dim $F \leq 1$ and $\operatorname{Hom}_{\Lambda}(F, \Lambda)=0, \operatorname{so} \underline{\operatorname{dim}} \tau_{\Lambda}(F)=$ $\phi_{\Lambda}(\underline{\operatorname{dim}} F)$, but on the other hand $F^{\prime}$, as a quasi-simple non-homogeneous object in $\mathcal{X}_{d}$, is the unique up to isomorphism indecomposable $\Lambda$-module with the dimension vector equal to $\underline{\operatorname{dim}} F^{\prime}$ (see [19,2.4] and [14]). Now we check case by case for each $\left(u ;\left(u_{i}\right)\right) \in \Theta^{(d)}$, with $d=p, \ldots, 2 p-1$ (resp. $d=p+1, \ldots, 2 p$, if $\left.\mathbf{p}=(2,3,6)\right)$, that $\phi_{\Lambda}\left(\underline{\operatorname{dim}} M^{\left(u ;\left(u_{i}\right)\right)}\right)=\underline{\operatorname{dim}} M^{\left(u^{\prime} ;\left(u_{i}^{\prime}\right)\right)}$, for some $\left(u^{\prime} ;\left(u_{i}^{\prime}\right)\right) \in \Theta^{(d)},\left(u^{\prime} ;\left(u_{i}^{\prime}\right)\right) \neq\left(u ;\left(u_{i}\right)\right)$. Next, using the induction arguments based on $(*)$ and the fact that $\phi_{\Lambda}(11)=11$, we show that our claim holds for all $d$. In this way the proof of the lemma is complete.

Proof of Theorem 5.2 Fix $q$ as in the statement. We define inductively a sequence $B_{s}, \ldots, B_{0}$ of $k[\xi] \otimes_{k} \Lambda$-modules with the following properties: for every $i$, the module $B_{i}$ belongs to $\bmod _{k[\xi]} \Lambda$ and its localization $\left(B_{i}\right)_{f}$ yields a parametrizing bimodule for $\mathcal{X}_{\mu_{i}^{\prime}}^{0}$; moreover, $B_{0}=B^{(q)}$. Without loss of generality we can assume that $q \notin \mathbb{Z}$, since otherwise $s=0$ and the assertion trivially holds.

We set $B_{s}:=B^{\left(\mu_{s}^{\prime}\right)}\left(\mu_{s}^{\prime} \in \mathbb{Z}_{\geq p}\right.$, so $B^{\left(\mu_{s}^{\prime}\right)}$ is known!; cf.Proposition 5.2). It is clear that $B_{s}$ satisfies all the required conditions. Assume that $B_{i}$ with the required properties is already defined, where $1 \leq i \leq s$. Note that by Proposition 5.2 we have $\mu_{i}<\mu_{i}^{\prime}$ and $\mu_{i} \in \mathbb{Z}_{\geq p}\left(\right.$ resp. $\mu_{i} \in \mathbb{Z}_{\geq 7}$, if $\left.\mathbf{p}=(2,3,6)\right)$, since $\mu^{\prime \prime} \in \mathbb{Q}_{\geq p}\left(\right.$ resp. $\mu^{\prime \prime} \in \mathbb{Q}_{\geq 7} ; \mu^{\prime \prime} \notin \mathbb{Z}$ !). Then, by Lemma $3.3, \mathcal{F}^{\left(\mu_{i}\right)}$ is a semisimple category of the type $\mathcal{F}(\mathcal{T})$, where $\mathcal{T}$ 
is a stable tube in $\mathcal{X}_{\mu_{i}^{\prime}}$ of the maximal rank $\rho_{\mathcal{T}}=p$ (hence, $m_{\mathcal{T}}=1$ ). Moreover, the category $\tilde{\mathcal{F}}^{\left(\mu_{i}\right)}$ is $R$-semisimple, for $R=k[\xi]$, the universal extension of $B_{i}$ with respect to $\widetilde{\mathcal{F}}^{\left(\mu_{i}\right)}$ exists, the $k[\xi] \otimes_{k} \Lambda$-module $\Upsilon_{\mu_{i}}\left(B_{i}\right)=\Upsilon_{\widetilde{\mathcal{F}}\left(\mu_{i}\right)}\left(B_{i}\right)$ belongs to $\bmod _{R} \Lambda$ and its localization $\left(\Upsilon_{\mu_{i}}\left(B_{i}\right)\right)_{f}$ is a parametrizing bimodule for $\mathcal{X}_{\mu_{i-1}^{\prime}}^{0}$ (see Theorem 3.2, Corollary 4.12 and Remark 4.12). Now, we set $B_{i-1}:=\Upsilon_{\mu_{i}}\left(B_{i}\right)$.

In this way the construction of the sequence $B_{s}, \ldots, B_{0}$ of modules is complete. It is clear that $B_{0}=B^{(q)}$. Consequently, the module $B^{(q)}$ satisfies all the properties from the assertion.

Corollary For any $i$, we have $B^{\left(\mu_{i}^{\prime}\right)}=B_{i}$, where $B_{i}$ are constructed for $q=\mu^{\prime \prime}$ as above.

Proof Follows immediately by Remark 5.2 (c).

To prove the main result, Theorem 3.3, we need the precise constructive description of the sequences $\mu^{\prime \prime}$, for $\mu^{\prime \prime} \in \mathbb{Q}_{\geq p} \backslash \mathbb{Z}$ (cf. Remark 5.2 (a) and (b)). For this aim more detailed analysis of the inductive proof of Proposition 5.2 is necessary.

Let $a\left(\mu^{\prime \prime}\right):=\left(a_{0}, \ldots, a_{n+1}, a_{n+2}\right)$, where $a_{0}=a, a_{1}=b, a_{n+1}=1, a_{n+2}=0$, and $c\left(\mu^{\prime \prime}\right):=\left(c_{1}, \ldots, c_{n+1}\right)$ be the two sequences constructed for $\mu^{\prime \prime}$ as in 3.3 , where $\mu^{\prime \prime}=\frac{a}{b}$ is the fraction presentation such that $\operatorname{gcd}(a, b)=1$. Note that $\mu_{0}^{\prime}=\mu^{\prime \prime}=$ $c_{1}+\frac{a_{2}}{a_{1}}$ and $n \geq 1$, since $\mu^{\prime \prime} \notin \mathbb{Z}$, so $a_{2}>0$. Moreover, $s=s\left(\mu^{\prime \prime}\right) \geq 1, \mu_{1}=c_{1}$ and $\mu_{1}^{\prime}=$ $\frac{a_{0}-c_{1} a_{2}}{a_{1}-a_{2}}=c_{1}+\frac{a_{2}}{a_{1}-a_{2}}$ (see Corollary 5.1). Now the following three cases are possible:

$1^{o} \quad a_{2}=a_{1}-a_{2}$, equivalently, $a_{1}=2 a_{2}$ : Then $c_{2}=2, a_{3}=0$ and $\mu_{1}^{\prime}=c_{1}+1 \in \mathbb{Z}_{\geq p}$.

$2^{o} \quad a_{2}<a_{1}-a_{2}$, equivalently, $2 a_{2}<a_{1}$ : Then quo $a_{1-a_{2}}\left(a_{0}-c_{1} a_{2}\right)=c_{1}$ and $\operatorname{rem}_{a_{1}-a_{2}}\left(a_{0}-c_{1} a_{2}\right)=a_{2}$, hence, by the formula from Corollary $5.1 \mu_{2}=c_{1}$ and $\mu_{2}^{\prime}=\frac{\left(a_{0}-c_{1} a_{2}\right)-c_{1} a_{2}}{\left(a_{1}-a_{2}\right)-a_{2}}=\frac{a_{0}-2 c_{1} a_{2}}{a_{1}-2 a_{2}}=c_{1}+\frac{a_{2}}{a_{1}-2 a_{2}}$.

$3^{o} \quad a_{2}>a_{1}-a_{2}$, equivalently, $2 a_{2}>a_{1}$ : Then $c_{2}=1$ and $a_{1}-a_{2}=a_{1}-c_{2} a_{2}=a_{3}$, so $a_{3}>0$. Hence $a_{0}-c_{1} a_{2}=c_{1} a_{3}+a_{2}=\left(c_{1}+c_{3}\right) a_{3}+a_{4}$, so from the formula for $\mu_{1}^{\prime}$ we have $\mu_{1}^{\prime}=c_{1}+c_{3}+\frac{a_{4}}{a_{3}}$. Moreover quo $a_{a_{1}-a_{2}}\left(a_{0}-c_{1} a_{2}\right)=c_{1}+c_{3}$ and $\operatorname{rem}_{a_{1}-a_{2}}\left(a_{0}-c_{1} a_{2}\right)=a_{4}$. Consequently, $\mu_{2}=c_{1}+c_{3}$ and $\mu_{2}^{\prime}=c_{1}+c_{3}+\frac{a_{4}}{a_{3}-a_{4}}$, if $a_{4} \neq 0$. Note that $\mu_{1}^{\prime}=c_{1}+c_{3} \in \mathbb{Z}_{\geq p}$, in case $a_{4}=0$.

Now repeating inductively the arguments above, one can give the close formulas for all members of the sequences $\mu^{\prime \prime}$ and the formulas reconstructing $\mu_{0}^{\prime}=$ $\mu^{\prime \prime}, \ldots \mu_{s-1}^{\prime}$ from $\mu_{s}^{\prime} \in \mathbb{Z}_{\geq p}$. To present them precisely we fix some notation.

For any $d \in \mathbb{N}$, we denote by $\varsigma_{(d)}$ the map $\varsigma_{M_{d}}: \overline{\mathbb{Q}} \rightarrow \overline{\mathbb{Q}}$, where $M_{d}:=\left[\begin{array}{cc}1+d & -d^{2} \\ 1 & 1-d\end{array}\right]$, i.e. given by $\varsigma_{(d)}\left(\mu^{\prime}\right):=\mu^{\prime \prime}\left(d, \mu^{\prime}\right)=\frac{d x-d^{2} y+x}{x-d y+y}$, for $\mu^{\prime}=\frac{x}{y} \in \overline{\mathbb{Q}}$.

Remark If $x=d y+z$ then $\varsigma_{(d)}\left(\frac{x-l d z}{y-l z}\right)=\frac{x-(l-1) d z}{y-(l-1) z}$, for $l \in \mathbb{N}$. 
Proposition For any $\mu^{\prime \prime} \in \mathbb{Q}_{\geq p}$ the following formula holds

$$
\mu^{\prime \prime}= \begin{cases}\left(\varsigma_{\left(c_{1}\right)}^{c_{2}} \circ \cdots \circ \varsigma_{\left(c_{1}+c_{3}+\cdots+2 m-1\right)}^{c_{2 m}}\right)\left(c_{1}+c_{3}+\cdots+c_{2 m+1}\right) & \text { if } n=2 m, \\ \left(\varsigma_{\left(c_{1}\right)}^{c_{2}} \circ \cdots \circ \varsigma_{\left(c_{1}+c_{3}+\cdots+2 m-1\right)}^{c_{2 m}} \circ \varsigma_{\left(c_{1}+c_{3}+\cdots+c_{2 m+1}\right)}^{c_{2(m+1}-1}\right) & \text { if } n=2 m+1, \\ \times\left(c_{1}+c_{3}+\cdots+c_{2 m+1}+1\right) & \end{cases}
$$

where $c\left(\mu^{\prime \prime}\right)=\left(c_{1}, \ldots, c_{n+1}\right)$ (cf. Theorem 3.3). Moreover, $s\left(\mu^{\prime \prime}\right)=c_{0}+c_{2} \cdots+c_{2 m}$, if $n=2 m$; respectively, $s\left(\mu^{\prime \prime}\right)=c_{0}+c_{2} \cdots+c_{2(m+1)}-1$, if $n=2 m+1$, where $c_{0}:=0$, and for any $i \leq s\left(\mu^{\prime \prime}\right)$ such that $c_{0}+c_{2}+\cdots+c_{2 j}+1 \leq i \leq c_{0}+c_{2}+\cdots+c_{2(j+1)}$, we always have $\mu_{i}=c_{1}+c_{3} \cdots+c_{2 j+1}$, and

$$
\begin{aligned}
\mu_{i}^{\prime} & =c_{1}+c_{3}+\cdots+c_{2 j+1}+\frac{a_{2(j+1)}}{a_{2 j+1}-\left(i-\left(c_{0}+c_{2}+\cdots+c_{2 j}\right)\right) a_{2(j+1)}} \\
& =\left(\varsigma_{\left(c_{1}+c_{3} \cdots+c_{2 j+1}\right)}^{c_{2}+\cdots+c_{2(j+1)}-i} \circ \cdots \circ \varsigma_{\left(c_{1}+c_{3} \cdots+c_{2 m-1}\right)}^{c_{2 m}}\right)\left(c_{1}+c_{3}+\cdots+c_{2 m+1}\right),
\end{aligned}
$$

if $n=2 m$; respectively,

$$
\begin{aligned}
\mu_{i}^{\prime} & =c_{1}+c_{3}+\cdots+c_{2 j+1}+\frac{a_{2(j+1)}}{a_{2 j+1}-\left(i-\left(c_{0}+c_{2}+\cdots+c_{2 j}\right)\right) a_{2(j+1)}} \\
& =\left(\varsigma_{\left(c_{1}+c_{3} \cdots+c_{2 j+1}\right)}^{c_{2}+\cdots+c_{2(j+1)}-i} \circ \cdots \circ \varsigma_{\left(c_{1}+c_{3} \cdots+c_{2 m-1}\right)}^{c_{2 m}} \circ \varsigma_{\left(c_{1}+c_{3} \cdots+c_{2 m+1}\right)}^{c_{2(m+1)}-1}\right)\left(c_{1}+c_{3}+\cdots+c_{2 m+1}+1\right), \\
\text { if } n & =2 m+1, \text { where } a\left(\mu^{\prime \prime}\right)=\left(a_{0}, a_{1}, \ldots, a_{n+1}, a_{n+2}\right) .
\end{aligned}
$$

Proof We prove the assertion by induction on $n:=n\left(\mu^{\prime \prime}\right)$. The case $n=0$ is obvious, since then $m=0, a_{2}=0$ and $\mu^{\prime \prime}=c_{1} \in \mathbb{Z}$, so all the formulas trivially hold. If $n=1$ then $m=0, a_{3}=0, \mu^{\prime \prime} \in \mathbb{Q}_{\geq p} \backslash \mathbb{Z}$ and $s:=s\left(\mu^{\prime \prime}\right) \geq 1$. Applying inductively the arguments as in $2^{o}$ and $1^{o}$, we infer that $s=c_{2}-1$, moreover, $\mu_{i}=c_{1}$ and $\mu_{i}^{\prime}=$ $c_{1}+\frac{a_{2}}{a_{1}-i a_{2}}$, for every $i=1, \ldots, c_{2}-1$. (Note that $\mu_{c_{2}-1}^{\prime}=c_{1}+1$, since $a_{1}=c_{2} a_{2}$ ). Consequently, in this situation all the required formulas follow easily (cf. Section 5.1 and Remark).

Assume now that $n \geq 2$. Then the formulas for $\mu_{i}$ and $\mu_{i}^{\prime}$, where $i=1, \ldots, c_{2}-$ 1 , remain still valid, but $a_{3} \neq 0$, so $a_{1}-c_{2} a_{2}>0$; hence, $\mu_{c_{2}}=c_{1}$ and $\mu_{c_{2}}^{\prime}=c_{1}+$ $\frac{a_{2}}{a_{1}-c_{2} a_{2}}=c_{1}+c_{3}+\frac{a_{4}}{a_{3}}$, since $a_{1}-c_{2} a_{2}=a_{3}$ and $a_{2}=c_{3} a_{3}+a_{4}$ (cf. $2^{o}$ and $3^{o}$ ). Note that we have $\mu_{i}^{\prime}=\varsigma_{\left(c_{1}\right)}^{c_{2}-i}\left(\mu_{c_{2}}^{\prime}\right)$, for every $i=1, \ldots, c_{2}$ and also for $i=0$ (see Section 5.1 and Remark). In particular, if $a_{4}=0$, then $n=2, m=1$ and $\mu_{c_{2}}^{\prime}=c_{1}+c_{3} \in \mathbb{Z}_{\geq p}$, so all the formulas hold true.

Consider the case $a_{4} \neq 0$. Set $a^{\star}:=\left(c_{1}+c_{3}\right) a_{3}+a_{4}, b^{\star}:=a_{3}$ and $\left(\mu^{\prime \prime}\right)^{\star}:=\frac{a^{\star}}{b^{\star}}$. Then $\left(\mu^{\prime \prime}\right)^{\star}=\mu_{c_{2}}^{\prime} \notin \mathbb{Z}$, so $s^{\star}=s-c_{2} \geq 1$, where $s^{\star}:=s\left(\left(\mu^{\prime \prime}\right)^{\star}\right)$, and $n^{\star}=n-2$, where $n^{\star}:=n\left(\left(\mu^{\prime \prime}\right)^{\star}\right)$. Moreover, the sequence $\left(\mu^{\prime \prime}\right)^{\star}=\left(\mu_{i^{\star}}^{\star},\left(\mu^{\prime}\right)_{i^{\star}}\right)_{i^{\star}=1, \ldots, s^{\star}}$ is given by the equalities (i): $\mu_{i^{\star}}^{\star}=\mu_{c_{2}+i^{\star}}$ and $\left(\mu^{\prime}\right)_{i^{\star}}^{\star}=\mu_{c_{2}+i^{\star}}^{\prime}(\mathrm{cf}$. Remark 5.2(c)). Observe that $\operatorname{gcd}\left(a^{\star}, b^{\star}\right)=\operatorname{gcd}\left(a_{3}, a_{4}\right)=1$ and the sequences $a\left(\left(\mu^{\prime \prime}\right)^{\star}\right)=\left(a_{1}^{\star}, \ldots, a_{n^{\star}+2}^{\star}\right)$ and $c\left(\left(\mu^{\prime \prime}\right)^{\star}\right)=\left(c_{1}^{\star}, \ldots, c_{n^{\star}+1}^{\star}\right)$ have the following shape (ii): $a_{0}^{\star}=\left(c_{1}+c_{3}\right) a_{3}+a_{4}, a_{1}^{\star}=$ $a_{3}, \ldots, a_{n}^{\star}=a_{n+2}$ and $c_{1}^{\star}=c_{1}+c_{3}, c_{2}^{\star}=c_{4}, \ldots, c_{n-1}^{\star}=c_{n+1}$.

Now we can close the proof. By the inductive assumption we infer the validity of the formulas as in the statement for members of the sequence $\left(\mu^{\prime \prime}\right)^{\star}$. They are actually expressed in terms of the sequences $a\left(\left(\mu^{\prime \prime}\right)^{\star}\right)$ and $c\left(\left(\mu^{\prime \prime}\right)^{\star}\right)$. Applying them together 
with the equalities (i) and (ii), and next combining with the formulas for $\mu_{i}$ and $\mu_{i}^{\prime}$, with $i \leq c_{2}$, from the beginning of the discussion for the case $n \geq 2$, we immediately obtain our assertion (cf. Section 5.1 and Remark).

Now we can prove the first part of Theorem 3.3.

Proof of Theorem 3.3(a) Fix $q$ as in the statement (a). Then by Theorem 5.2, the $k[\xi] \otimes_{k} \Lambda$-module $B^{(q)}:=\left(\Upsilon_{\mu_{1}} \circ \cdots \circ \Upsilon_{\mu_{s}}\right)\left(B^{\left(\mu_{s}^{\prime}\right)}\right)$ is well defined, belongs to $\bmod _{k[\xi]} \Lambda$ and its localization $\left(B^{(q)}\right)_{f}$ yields a parametrizing bimodule for $\mathcal{X}_{q}^{0}$, where $\mu_{s}^{\prime}$ and $\mu_{1}, \ldots, \mu_{s}$ are elements of the sequences forming $\mu^{\prime \prime}$, for $\mu^{\prime \prime}:=q$. Now inserting into the formula above the values for $\mu_{s}^{\prime}$ and $\mu_{1}, \ldots, \mu_{s}$, given in Proposition 5.3, we immediately obtain the assertion (a) of Theorem 3.3.

The assertion (b) of Theorem 3.3 has a quite different character than (a). It covers a very specific case and can be treated as a "patch" which fills in the gap being a result of certain nonregularity of tubular structure of $\bmod \Lambda$, for the weight sequence $\mathbf{p}=(2,3,6)$. To prove (b) we will apply the technique which is in some sense dual to (co)universal extensions of bimodules. In our situation this technique occurs to be less complicated than the previous one. Therefore we treat this case more loosely, sketching only briefly some parts of our exposition and omitting sometimes the technical details.

Let $\Lambda=k Q / I$ be a (finite dimensional) $k$-algebra of a bound quiver $(Q, I)$ and $R$ a commutative $k$-algebra. Then for any $R \otimes_{k} \Lambda$-module $B$ in $\bmod _{R} \Lambda$ and full subcategory $\mathcal{F}$ formed by pairwise nonisomorphic objects in $\bmod _{R} \Lambda$, by

$$
\operatorname{can}_{B}^{\mathcal{F}}: \bigoplus_{F \in \mathrm{ob} \mathcal{F}} \operatorname{Hom}_{R \otimes \Lambda}(F, B) \otimes_{R} F \longrightarrow B
$$

we always mean the canonical $R \otimes_{k} \Lambda$-homomorphism, given by the mappings $\varphi \otimes$ $f_{x} \mapsto \varphi_{x}\left(f_{x}\right)$, for $F \in$ ob $\mathcal{F}$ and $x \in Q_{0}$, where $\varphi=\left(\varphi_{x}\right)_{x \in Q_{0}} \in \operatorname{Hom}_{R \otimes \Lambda}(F, B)$ and $f_{x} \in F_{x}$, and by $\mathcal{K}_{\mathcal{F}}(B)$ we denote the $R \otimes_{k} \Lambda$-module

$$
\mathcal{K}_{\mathcal{F}}(B):=\operatorname{Ker}\left(\operatorname{can}_{B}^{\mathcal{F}}\right) .
$$

The following analog of Theorem 3.1 will play a basic role.

Theorem Let $\Lambda=k Q /$ I be a finite dimensional $k$-algebra of a bound quiver $(Q, I)$, $R=k[\xi]_{f}$ the localization of $k[\xi]$, where $f \in k[\xi]$ is a nonzero polynomial, and $\mathcal{F}:=$ $\left\{F_{1}, \ldots, F_{\rho}\right\}$ a subcategory in $\bmod \Lambda$. Then for any $R \otimes_{k} \Lambda$-module $B$ from $\bmod _{R} \Lambda$ satisfying the following two conditions:

- the sequence $\left(\operatorname{dim}_{k} \operatorname{Hom}_{\Lambda}\left(F_{i}, B(a)\right)\right)_{a \in k_{f}}$ is constant for every $i=1, \ldots, \rho$,

- the canonical map $\operatorname{can}_{B(a)}^{\mathcal{F}}: \bigoplus_{i=1}^{\rho} \operatorname{Hom}_{\Lambda}\left(F_{i}, B(a)\right) \otimes_{k} F_{i} \rightarrow B(a)$ in $\bmod \Lambda$ is surjective for all $a \in k_{f}$,

the canonical map $\operatorname{can}_{B}^{\widetilde{\mathcal{F}}}: \bigoplus_{i=1}^{\rho} \operatorname{Hom}_{R \otimes \Lambda}\left(\tilde{F}_{i}, B\right) \otimes_{R} \tilde{F}_{i} \longrightarrow B$ in $\bmod R \otimes_{k} \Lambda$ is surjective, $\mathcal{K}_{\widetilde{\mathcal{F}}}(B)$ belongs to $\bmod _{R} \Lambda$ and

$$
\left(\mathcal{K}_{\widetilde{\mathcal{F}}}(B)\right)(a, l) \cong \mathcal{K}_{\mathcal{F}}(B(a, l))
$$


in $\bmod \Lambda$, for all $a \in k_{f}$ and $l \in \mathbb{N}_{+}$, where $\tilde{\mathcal{F}}:=\left\{\tilde{F}_{1}, \ldots, \tilde{F}_{\rho}\right\}$ and $\tilde{F}_{i}=R \otimes_{k} F_{i}$ for every $i$.

The proof of this result needs some preparation and will be given in Section 5.5.

We start with the information on behaviour of homomorphism spaces for $R \otimes_{k} \Lambda$ modules from $\bmod _{R} \Lambda$ under the "change of coefficients" (cf. Section 4.11).

Proposition Let $R=k[\xi]_{f}$ be a localization of the polynomial ring $k[\xi]$, where $f \in$ $k[\xi]$, and $B, B^{\prime}$ a pair of $R \otimes_{k} \Lambda$-modules from $\bmod _{R} \Lambda$. If the dimension sequence

$$
\left(\operatorname{dim}_{k} \operatorname{Hom}_{\Lambda}\left(B(a), B^{\prime}(a)\right)\right)_{a \in k_{f}}
$$

is constant, then for $\left(B, B^{\prime}\right)$ the following condition holds:

(H2) $\breve{h}_{B, B^{\prime}}: \bar{R} \otimes_{R} \operatorname{Hom}_{R \otimes \Lambda}\left(B, B^{\prime}\right) \rightarrow \operatorname{Hom}_{\bar{R} \otimes \Lambda}\left(\bar{B}, \bar{B}^{\prime}\right)$ is a $\bar{R}$-isomorphism, for every algebra homomorphism $h: R \rightarrow \bar{R}$, where $\bar{B}=\bar{R} \otimes_{R} B$ and $\bar{B}^{\prime}=\bar{R} \otimes$ ${ }_{R} B^{\prime}$ (see Section 4.3).

Proof Without loss of generality we can assume as in Section 4.11 that $B, B^{\prime}$ belong to $\bmod _{R}^{\text {mat }} \Lambda$. Let $\mathcal{M}^{0}=\mathcal{M}^{0}\left(B, B^{\prime}\right) \in \mathbb{M}_{v_{1} \times v_{0}}(R)$ be a matrix as in Lemma 4.8. Since $R$ is a principle ideal domain, there exists a pair of invertible matrices $\mathcal{W} \in \mathrm{u}\left(\mathbb{M}_{v_{1} \times v_{1}}(R)\right)$ and $\mathcal{X} \in \mathrm{u}\left(\mathbb{M}_{v_{0} \times v_{0}}(R)\right)$ such that $\mathcal{W} \mathcal{M}^{0} \mathcal{X}=\mathcal{D}^{0}$, where $\mathcal{D}^{0} \in$ $\mathbb{M}_{v_{1} \times v_{0}}(R)$ is a diagonal matrix as in Corollary 4.9(b) with $d_{1}^{0}, \ldots, d_{r^{0}}^{0} \neq 0$ (see [20]). We first show that our assumption implies that $d_{1}^{0}, \ldots, d_{r^{0}}^{0} \in \mathrm{u}(R)$. For this aim we use the formula $\operatorname{Hom}_{\bar{R} \otimes \Lambda}\left(\bar{B}, \bar{B}^{\prime}\right) \cong \bar{R}^{v_{0}-r^{0}} \oplus \operatorname{ann}_{\bar{R}} \bar{d}_{1}^{0} \oplus \cdots \oplus \operatorname{ann}_{\bar{R}} \bar{d}_{r^{0}}^{0}$ in $\bmod \bar{R}$, which holds for any ring homomorphism $h: R \rightarrow \bar{R}$ (cf. Corollary 4.9(a)). Applying it for $\bar{R}=R /(\xi-a) \cong k$ and $h=\pi_{a}: R \rightarrow k$, for all $a \in k_{f}$ as in Section 4.11, we obtain our claim (see [Dowbor P. and Mróz A., 2013, On the normal forms of modules with respect to parametrizing bimodules, unpublished] for details).

To prove the main assertion observe that $R^{v_{0}}=\bigoplus_{i=1}^{v_{0}} R \mathcal{X}^{(i)}$ and $\bar{R}^{v_{0}}=$ $\bigoplus_{i=1}^{v_{0}} R \overline{\mathcal{X}}^{(i)}$, moreover, $\operatorname{Hom}_{R \otimes \Lambda}\left(B, B^{\prime}\right)=\bigoplus_{i=r^{0}+1}^{v_{0}} R \mathcal{X}^{(i)}$ and $\operatorname{Hom}_{\bar{R} \otimes \Lambda}\left(\bar{B}, \bar{B}^{\prime}\right)=$ $\bigoplus_{i=r^{0}+1}^{v_{0}} R \overline{\mathcal{X}}^{(i)}$, since $d_{1}^{0}, \ldots, d_{r^{0}}^{0} \in \mathrm{u}(R)$ (see also Lemmata 4.8(b) and 4.9(b)). The standard isomorphism $\breve{h}: \bar{R} \otimes_{R} R^{v_{0}} \rightarrow \bar{R}^{v_{0}}$ sends $1 \otimes \mathcal{X}^{(i)}$ to $\overline{\mathcal{X}}^{(i)}$, so $\breve{h}^{h o m}{ }_{B, B^{\prime}}$ is the restriction of $\breve{h}$ to the respective direct summand. Consequently, $\breve{h}^{\text {hom }}{ }_{B, B^{\prime}}$ is an $R$ isomorphism.

Remark $\operatorname{Hom}_{R \otimes \Lambda}\left(B, B^{\prime}\right)$ is always a finitely generated free $R$-module (cf. (E1) in Section 4.10).

In the proof of Theorem 5.4 we use also the following fact.

Lemma Let $R$ be as above. Then for a matrix $\mathcal{N} \in \mathbb{M}_{v_{2}^{\prime} \times v_{1}^{\prime}}(R)$, the $R$-homomorphism $\mathcal{N} \cdot: R^{v_{1}^{\prime}} \rightarrow R^{v_{2}^{\prime}}$ is surjective if and only if $\mathcal{N}(a) \cdot: k^{v_{1}^{\prime}} \rightarrow k^{v_{2}^{\prime}}$ is surjective for every $a \in k_{f}$, where $\mathcal{N}(a):=\overline{\mathcal{N}} \in \mathbb{M}_{v_{2}^{\prime} \times v_{1}^{\prime}}(k)$, for $h=\pi_{a}: R \rightarrow k$ as above (see Section 4.9 ). 
Proof Let $\mathcal{D} \in \mathbb{M}_{v_{2}^{\prime} \times v_{1}^{\prime}}(R)$ be a diagonal matrix with $d_{1}, \ldots, d_{r} \neq 0$ of the shape as in Corollary $4.9(\mathrm{a})$ (we replace only $v_{i}$ by $v_{i}^{\prime}$ ) such that $\mathcal{D}=\mathcal{U} \mathcal{N} \mathcal{V}$, where $\mathcal{U} \in$ $\mathrm{u}\left(\mathbb{M}_{v_{2}^{\prime} \times v_{2}^{\prime}}(R)\right)$ and $\mathcal{V} \in \mathrm{u}\left(\mathbb{M}_{v_{1}^{\prime} \times v_{1}^{\prime}}(R)\right)$ is a pair of invertible matrices (the existence of $\mathcal{D}, \mathcal{U}, \mathcal{V}$ for $\mathcal{N}$ follow by [20]). Observe that $\mathcal{N}$. in surjective if and only if $r=v_{2}^{\prime}$ and $d_{1}, \ldots, d_{r} \in \mathrm{u}(R)$, since $R^{v_{2}^{\prime}} / \operatorname{Im} \mathcal{N} . \cong R^{v_{2}^{\prime}-r} \oplus R /\left(d_{1}\right) \oplus \cdots \oplus R /\left(d_{r}\right)$. Moreover, similarly $\mathcal{N}(a)$, for $a \in k_{f}$, is surjective if and only if $d_{1}(a), \ldots, d_{r}(a) \neq 0$ and $r=v_{2}^{\prime}$. Now the assertion follows from the fact that $d_{i} \in \mathrm{u}(R)$ if and only if $\pi_{a}\left(d_{i}\right)=d_{i}(a) \neq 0$, for every $a \in k_{f}$.

Proof of Theorem 5.4 W start by constructing a $\Lambda$-isomorphism

$$
\psi=\psi_{(a, l)}: \bar{R} \otimes_{R}\left(\bigoplus_{i=1}^{\rho} \operatorname{Hom}_{R \otimes \Lambda}\left(\tilde{F}_{i}, B\right) \otimes_{R} \tilde{F}_{i}\right) \rightarrow \bigoplus_{i=1}^{\rho} \operatorname{Hom}_{\Lambda}\left(F_{i}, B(a, l)\right) \otimes_{k} F_{i}
$$

where $\bar{R}=k[\xi] /(\xi-a)^{l}$ for $(a, l) \in k_{f} \times \mathbb{N}_{+}$. The map $\psi$ is given by the composition of the following isomorphisms:

$$
\begin{aligned}
\bar{R} \otimes_{R}\left(\bigoplus_{i=1}^{\rho} \operatorname{Hom}_{R \otimes \Lambda}\left(\tilde{F}_{i}, B\right) \otimes_{R} \tilde{F}_{i}\right) & \cong \bigoplus_{i=1}^{\rho} \bar{R} \otimes_{R} \operatorname{Hom}_{R \otimes \Lambda}\left(\tilde{F}_{i}, B\right) \otimes_{k} F_{i} \\
& \cong \bigoplus_{i=1}^{\rho} \operatorname{Hom}_{\bar{R} \otimes \Lambda}\left(\bar{R} \otimes_{R} \tilde{F}_{i}, \bar{R} \otimes_{R} B\right) \otimes_{k} F_{i} \\
& \cong \bigoplus_{i=1}^{\rho} \operatorname{Hom}_{\bar{R} \otimes \Lambda}\left(\bar{R} \otimes_{k} F_{i}, \bar{R} \otimes_{R} B\right) \otimes_{k} F_{i} \\
& \cong \bigoplus_{i=1}^{\rho} \operatorname{Hom}_{\Lambda}\left(F_{i}, \operatorname{Hom}_{\bar{R}}\left(\bar{R}, \bar{R} \otimes_{R} B\right)\right) \otimes_{k} F_{i} \\
& \cong \bigoplus_{i=1}^{\rho} \operatorname{Hom}_{\Lambda}\left(F_{i}, \bar{R} \otimes_{R} B\right) \otimes_{k} F_{i}
\end{aligned}
$$

where the first and the third isomorphisms are result of the definition of $\tilde{F}_{i}$, the second one is induced by the homomorphisms $\breve{h}^{\text {hom }} \tilde{F}_{i, B} \otimes \mathrm{id}_{F_{i}}$ for the canonical algebra projections $h: R \rightarrow \bar{R}$ (under our assumption all $\breve{h}^{\text {hom }} \tilde{F}_{i, B}$ are isomorphisms by Proposition 5.5), the fourth one is induced by the appropriate version of the classical adjunction formula and the fifth by the identification $\operatorname{Hom}_{\bar{R}}(\bar{R}, M) \cong M$, for $\bar{R}$-module $M$.

It is not hard to check that the value of $\psi$ on the typical element $s \otimes \varphi \otimes(r \otimes f) \in$ $\bar{R} \otimes_{R} \operatorname{Hom}_{R \otimes \Lambda}\left(\tilde{F}_{i}, B\right) \otimes_{R} \tilde{F}_{i}$, where $s \in \bar{R}, \varphi \in \operatorname{Hom}_{R \otimes \Lambda}\left(\tilde{F}_{i}, B\right), r \in R$ and $f \in F_{i}$, is equal to $\varphi_{s, r}^{\prime} \otimes f \in \operatorname{Hom}_{\Lambda}\left(F_{i}, B(a, l)\right) \otimes_{k} F_{i}$, where $\varphi_{s, r}^{\prime}\left(f^{\prime}\right)=s \bar{r} \otimes \varphi\left(1 \otimes f^{\prime}\right)$, for $f^{\prime} \in$ $F_{i}$. Consequently, we have

$$
\bar{R} \otimes_{R} \operatorname{can}_{B}^{\widetilde{\mathcal{F}}}=\operatorname{can}_{B(a, l)}^{\mathcal{F}} \circ \psi
$$

since $s \otimes \varphi(r \otimes f)=s \otimes r \varphi(1 \otimes f)=\varphi_{s, r}^{\prime}(f)$. In particular, by the second assertion we infer that $\bar{R} \otimes_{R} \operatorname{can}_{B}^{\widetilde{\mathcal{F}}}$ is an $\Lambda$-epimorphism for each pair $(a, 1)$, where $a \in k_{f}$. Then, by Lemma $5.5, \operatorname{can}_{B}^{\widetilde{\mathcal{F}}}$ is an epimorphism of $\operatorname{modules}$ from $\bmod _{R} \Lambda$, so it splits 
over $R$ and $\mathcal{K}_{\widetilde{\mathcal{F}}}(B)$ belongs to $\bmod _{R} \Lambda$. Hence, for every $(a, l) \in k_{f} \times \mathbb{N}_{+}$we have $\left(\mathcal{K}_{\widetilde{\mathcal{F}}}(B)\right)(a, l) \cong \operatorname{Ker}\left(\bar{R} \otimes_{R} \operatorname{can}_{B}^{\widetilde{\mathcal{F}}}\right)$ and by $(*)$ we obtain the $\Lambda$-isomorphisms

$$
\left(\mathcal{K}_{\widetilde{\mathcal{F}}}(B)\right)(a, l) \cong \mathcal{K}_{\mathcal{F}}(B(a, l)) .
$$

In this way the proof is complete.

Proof of Theorem 3.3(b) We start by some general considerations. Let $\Lambda, \mu, \mathcal{F}=$ $\mathcal{F}(\mathcal{T})$ and $\mathcal{L}$ be as in the proof of Theorem 3.2. (We keep also the all other necessary notations from Section 4.12.) One can show that for an indecomposable $X$ in $\operatorname{coh} \mathbb{X}$ such that $\mu(X)>\mu+\frac{1}{r^{2} m}$, the canonical map can ${ }_{X}^{\mathcal{F}}$ of sheaves, defined in an analogous way as in 5.4, is an epimorphism in coh $\mathbb{X}$ and we have the isomorphism

$$
\mathcal{L}(X) \cong \mathcal{K}_{\mathcal{F}}(X)
$$

where $\mathcal{K}_{\mathcal{F}}(X):=\operatorname{Ker}\left(\operatorname{can}_{X}^{\mathcal{F}}\right)$ (a slight generalization of [16, Corollary 5.2.2]). Now, using $(*)$ and proceeding analogously as in Section 4.12, we compute precisely $\mu(\mathcal{L}(X))$, for a fixed $X$ in $\mathcal{C}_{\dot{\mu}^{\prime}}$, where $\dot{\mu}^{\prime}>\mu+\frac{1}{r^{2} m}$. Since $\mu<\dot{\mu}^{\prime}$ implies $\operatorname{Ext}^{1}\left(\tau^{j} F_{1}, X\right)=0$, we have

$$
\begin{aligned}
\sum_{j=1}^{\rho} \operatorname{dim}_{k} \operatorname{Hom}\left(\tau^{j} F_{1}, X\right) & =\frac{1}{m}\left(\sum_{j=1}^{p} \operatorname{dim}_{k} \operatorname{Hom}\left(\tau^{j} F_{1}, X\right)\right) \\
& =\frac{1}{m}\left|\begin{array}{cc}
\operatorname{rk} F_{1} & \text { rk } X \\
\operatorname{deg} F_{1} & \operatorname{deg} X
\end{array}\right|=\operatorname{lm}^{\prime}\left(r d^{\prime}-r^{\prime} d\right)
\end{aligned}
$$

Consequently, $\operatorname{deg} \mathcal{L}(X)=\operatorname{deg} \mathcal{K}_{\mathcal{F}}(X)=l m^{\prime}\left(r d^{\prime}-r^{\prime} d\right) \operatorname{deg} F_{1}-\operatorname{deg} X$ and $\operatorname{rk} \mathcal{L}(X)=$ rk $\mathcal{K}_{\mathcal{F}}(X)=\operatorname{lm}^{\prime}\left(r d^{\prime}-r^{\prime} d\right)$ rk $F_{1}-$ rk $X$; hence,

$$
\begin{aligned}
& \mu(\mathcal{L}(X))=\frac{\operatorname{deg} \mathcal{L}(X)}{\operatorname{rk} \mathcal{L}(X)}=\frac{\operatorname{lm^{\prime }}\left(r d^{\prime}-r^{\prime} d\right) m d-l m^{\prime} d^{\prime}}{l m^{\prime}\left(r d^{\prime}-r^{\prime} d\right) m r-l m^{\prime} r^{\prime}} \\
& =\frac{d m\left(r d^{\prime}-r^{\prime} d\right)-d^{\prime}}{r m\left(r d^{\prime}-r^{\prime} d\right)-r^{\prime}}\left(=: \dot{\mu}^{\prime \prime}\left(\mu, \dot{\mu}^{\prime} ; m\right)\right)
\end{aligned}
$$

In particular, we see that for any $\mu^{\prime}$ as above, $\mathcal{L}$ induces a functor $\mathcal{L}_{\mid}: \mathcal{C}_{\dot{\mu}^{\prime}} \longrightarrow \mathcal{C}_{\dot{\mu}^{\prime \prime}}$, where $\dot{\mu}^{\prime \prime}=\dot{\mu}^{\prime \prime}\left(\mu, \dot{\mu}^{\prime} ; m\right)$.

Assume that the inequalities (**) : $\mu \geq p, \dot{\mu}^{\prime}>\mu+\frac{1}{r^{2} m}$ and $\dot{\mu}^{\prime \prime} \geq p$ hold (hence $d, r, d^{\prime}, r^{\prime} \in \mathbb{Z}_{\geq 1}$ and $r m\left(r d^{\prime}-r^{\prime} d\right)>r^{\prime}$; note also that since $\dot{\mu}^{\prime \prime}=\mu-\delta-\delta^{2} \frac{1}{\dot{\mu}^{\prime}-\mu-\delta}$, where $\delta:=\frac{1}{r^{2} m}$, so the third inequality does not follow from the first two). Assume additionally that $\mathcal{T} \neq \mathcal{T}^{0}$, if $\mu=p$. Then $\mathcal{F}, \mathcal{C}_{\dot{\mu}^{\prime}}$ and $\mathcal{C}_{\dot{\mu}^{\prime \prime}}$ are contained in $\operatorname{coh}_{+} \mathbb{X}$ so they can be regarded as subcategories of $\bmod _{+} \Lambda$ and identified with $\Psi(\mathcal{F}), \mathcal{X}_{\dot{\mu}^{\prime}}$ and $\mathcal{X}_{\dot{\mu}^{\prime \prime}}$, respectively. Moreover, observe that via $\Psi_{\mid \operatorname{coh}_{+} \mathbb{X}}=\operatorname{Hom}(T,-)_{\mid \operatorname{coh}_{+} \mathbb{X}}$ we have also $\operatorname{can}_{X}^{\mathcal{F}}=\operatorname{can}_{\Psi(X)}^{\Psi(\mathcal{F})}$ and $\mathcal{K}_{\mathcal{F}}(X)=\mathcal{K}_{\Psi(\mathcal{F})}(\Psi(X))$; in particular, $\operatorname{can}_{\Psi(X)}^{\Psi(\mathcal{F})}$ is an epi$\operatorname{morphism} \operatorname{in} \bmod \Lambda$. Let $\tilde{\mathcal{F}}_{f}:=\left\{k[\xi]_{f} \otimes_{k} F_{1}, \ldots, k[\xi]_{f} \otimes_{k} F_{\rho}\right\}$ be a $k[\xi]_{f}$-semisimple category associated with with the semisimple category $\mathcal{F}=\mathcal{F}(\mathcal{T})$ formed by all quasi-simple objects in the stable tube $\mathcal{T}$ in $\mathcal{X}_{\mu}$. Now using the identifications above and repeating the arguments from the second part of Section 4.12, we can prove that if $k[\xi]_{f} \otimes_{k} \Lambda$-module $B$ in $\bmod _{k[\xi]_{f}} \Lambda$ is a parametrizing bimodule for $\mathcal{X}_{\dot{\mu}^{\prime}}^{0}$, then the 
$k[\xi]_{f} \otimes_{k} \Lambda$-module $\mathcal{K}_{\widetilde{\mathcal{F}}_{f}}(B)$ belongs to $\bmod _{k[\xi]_{f}} \Lambda$ and it is a parametrizing bimodule for $\mathcal{X}_{\dot{\mu}^{\prime \prime}}^{0}$, where $\dot{\mu}^{\prime \prime}$ is given as above, provided the triple $\left(\mu, \dot{\mu}^{\prime}, \dot{\mu}^{\prime \prime}\right)$ satisfies the inequalities (**). (We have to replace $\widetilde{\mathcal{F}}$ by $\widetilde{\mathcal{F}}_{f}$, the sequences $\left(\operatorname{dim}_{k} \operatorname{Ext}_{\Lambda}^{1}\left(B(a), F_{i}\right)\right)_{a \in k_{f}}$ by $\left(\operatorname{dim}_{k} \operatorname{Hom}_{\Lambda}\left(F_{i}, B(a)\right)\right)_{a \in k_{f}}$, the functors $\mathcal{R}, \Upsilon_{\mathcal{F}}$ and $\Upsilon_{\widetilde{\mathcal{F}}}$ by $\mathcal{L}, \mathcal{K}_{\mathcal{F}}$ and $\mathcal{K}_{\widetilde{\mathcal{F}}_{f}}$, respectively, and apply Theorem 5.4 together with the isomorphism $(*))$.

Next observe that for any fixed $\mu=\frac{d}{r} \in \overline{\mathbb{Q}}$, with $\operatorname{gcd}(r, d)=1$, the function $\dot{\zeta}$ : $\overline{\mathbb{Q}} \rightarrow \overline{\mathbb{Q}}$ defined by the mapping $\dot{\mu}^{\prime} \mapsto \dot{\mu}^{\prime \prime}\left(\mu, \dot{\mu}^{\prime} ; m\right):=\frac{d m(r x-d y)-x}{r m(r x-d y)-y}$, for $\dot{\mu}^{\prime}=\frac{x}{y} \in \overline{\mathbb{Q}}$, has the form $\dot{\zeta}=\varsigma_{M}$ for the matrix $N:=\left[\begin{array}{cc}-1+d m r & -d^{2} m \\ r^{2} m & -1-d m r\end{array}\right]$, and $\operatorname{det} N=1$. Hence, $\dot{\zeta}$ is a bijection with the inverse $\dot{\zeta}^{-1}=\varsigma_{N^{-1}}$, which in the case $m, r=1$ is given by the formula $\dot{\zeta}^{-1}\left(\frac{a}{b}\right)=\frac{d^{2} b-(1+d) a}{(d-1) b-a}$, for $\frac{a}{b} \in \overline{\mathbb{Q}}$ (see Section 2.1 ; independently note that $N=-M^{-1}$, so $\dot{\zeta}=\varsigma^{-1}$, which reflects the fact that $\mathcal{R}$ and $\mathcal{L}$ are mutually quasiinverse). Moreover, if $m, r=1$ and $\mu=8(=d)$ then elementary checking shows that for any $\frac{a}{b} \in \overline{\mathbb{Q}}$ such that $6<\frac{a}{b}<7$, with $a, b \in \mathbb{Z}_{\geq 1}$, we have $\dot{\mu}^{\prime}>\mu+\frac{1}{r^{2} m}=9$, where $\dot{\mu}^{\prime}:=\dot{\zeta}^{-1}\left(\frac{a}{b}\right)=\frac{64 b-9 a}{7 b-a}$. (In fact $\dot{\zeta}^{-1}\left(\frac{a}{b}\right)>d+1$ holds for all $d \in \mathbb{Z}_{\geq 8}$ and $d=8$ is the smallest integer satisfying this inequality).

Finally, let $\mathbf{p}=(2,3,6), \mu=\frac{8}{1}$ and $\mathcal{F}=\mathcal{F}^{(8)}$ (so $r=1, d=8, \rho=6=p, m=1$ ). Assume that given $q=\frac{a}{b} \in \mathbb{Q}$ such that $6<q<7$, the module $B^{(\hat{q})}$ in $\bmod _{k[\xi]} \Lambda$ has the property that $B:=\left(B^{(\hat{q})}\right)_{f}$ is a parametrizing bimodule for $\mathcal{X}_{\hat{q}}^{0}$, where $\hat{q}:=\frac{64 b-9 a}{7 b-a}$. Then for $\dot{\mu}^{\prime}:=\hat{q}$, we clearly have $\dot{\mu}^{\prime \prime}\left(\mu, \dot{\mu}^{\prime} ; m\right)=q$ and the triple $\left(\mu, \dot{\mu}^{\prime}, \dot{\mu}^{\prime \prime}\right)$ satisfies the inequalities (**). Consequently, the $k[\xi]_{f} \otimes_{k} \Lambda$-module $\mathcal{K}_{\widetilde{\mathcal{F}}_{f}}(B)$ from $\bmod _{k[\xi]_{f}} \Lambda$ is a parametrizing bimodule for $\mathcal{X}_{q}^{0}$. In this way the proof of Theorem 3.3 is complete, by the remark below (see also Remark 4.12(a)).

Remark Let $\dot{\mathcal{F}}:=\left\{k[\xi] \otimes_{k} F_{1}, \ldots, k[\xi] \otimes_{k} F_{\rho}\right\}, \widetilde{\mathcal{F}}_{f}:=\left\{k[\xi]_{f} \otimes_{k} F_{1}, \ldots, k[\xi]_{f} \otimes_{k} F_{\rho}\right\}$ be the categories associated with the semisimple category $\mathcal{F}=\mathcal{F}(\mathcal{T})$ formed by all quasi-simple objects in the stable tube $\mathcal{T}$ in $\mathcal{X}_{\mu}$, and $\dot{B}$ be a $k[\xi] \otimes_{k} \Lambda$-module from $\bmod _{k[\xi]} \Lambda$. Applying the arguments analogous to those from Corollaries 4.11 and 4.12, in particular Proposition 5.5, we have the following:

(a) If $(* * *)$ : the sequence $\left(\operatorname{dim}_{k} \operatorname{Hom}_{\Lambda}\left(F_{i}, \dot{B}(a)\right)\right)_{a \in k}$ is stable for every $i=1, \ldots, \rho$, then $\mathcal{K}_{\widetilde{\mathcal{F}}_{f}}(B) \cong\left(\mathcal{K}_{\dot{\mathcal{F}}}(\dot{B})\right)_{f}$ in $\bmod _{k[\xi]_{f}} \Lambda$ and $\left(\mathcal{K}_{\widetilde{\mathcal{F}}_{f}}(B)\right)(a, l) \cong$ $\left(\mathcal{K}_{\dot{\mathcal{F}}}(\dot{B})\right)(a, l) \cong \mathcal{K}_{\mathcal{F}}(\dot{B}(a, l))$ in $\bmod \Lambda$, for all $a \in k_{f}$ and $l \in \mathbb{N}_{+}$, where $B:=$ $(\dot{B})_{f}$.

(b) The condition $(* * *)$ always holds if $B$ is a parametrizing bimodule for $\mathcal{X}_{\dot{\mu}^{\prime}}^{0}$ and $\dot{B}(a)$ belong to $\mathcal{X}_{\dot{\mu}^{\prime}}$ for all $a \in \mathcal{Z}(f)$, provided $\left(\mu, \dot{\mu}^{\prime}, \dot{\mu}^{\prime \prime}\right)$ satisfies inequality $(* *)$. (Hence we also have that all $\left(\mathcal{K}_{\dot{\mathcal{F}}}(\dot{B})\right)(a)$ belong to $\mathcal{X}_{\dot{\mu}^{\prime \prime}}$, for $\left.a \in \mathcal{Z}(f)\right)$. Moreover, the functor $-\otimes_{k[\xi]} \mathcal{K}_{\dot{\mathcal{F}}}(\dot{B}): \bmod k[\xi] \rightarrow$ add $\mathcal{X}_{\dot{\mu}^{\prime \prime}}$ yields an injection between the respective sets of isoclasses of indecomposable objects, provided so does $-\otimes_{k[\xi]} \dot{B}: \bmod k[\xi] \rightarrow \operatorname{add} \mathcal{X}_{\dot{\mu}^{\prime}}$.

We finish the paper with an example which shows that the presented procedure (see Main Theorem 3.3, also Proposition 5.2 and Theorem 5.2) really yields an 
effective method for computing the matrix bimodule parametrizing all homogeneous indecomposable modules with a fixed slope.

Example Let $\Lambda=\Lambda((2,2,2,2), \lambda)$, for fixed $\lambda \in k, \lambda \neq 0,1$. Set $q:=\frac{18}{7} \geq p=2$. Then the sequences $c, a$ and $\mu^{\prime \prime}$ have the form $c=(2,1,1,3), a=(18,7,4,3,1,0)$, $\mu^{\prime \prime}=\left((2,3,3),\left(\frac{18}{7}, \frac{10}{3}, \frac{7}{2}, 4\right)\right)$, respectively. (Apply the definitions and Proposition $\overline{5.3}$, note also that $n=3, s=3)$. Now, by Theorem 3.3, we have

$$
B^{\left(\frac{18}{7}\right)}=\left(\Upsilon_{c_{1}}^{c_{2}} \circ \Upsilon_{c_{1}+c_{3}}^{c_{4}-1}\right)\left(B^{\left(c_{1}+c_{3}+1\right)}\right)=\left(\Upsilon_{2} \circ \Upsilon_{3}^{2}\right)\left(B^{(4)}\right)
$$

Let $R=k[\xi]_{f}$, for $f=\xi(\xi-1)(\xi-\lambda)$ (cf. 3.2). Below we present matrix bimodules $B$ from $\bmod _{R}^{\text {mat }} \Lambda$, in fact from $\bmod _{k[\xi]}^{\text {mat }} \Lambda$ (cf. Section 2.1 ), as $4 \times 2$ arrays, where $(i, j)$ th entry is an $R$-matrix $B_{\alpha_{j}^{(i)}}$ (see Sections 2.1 and 2.2). The $R$-semisimple categories $\widetilde{\mathcal{F}}^{(2)}$ and $\widetilde{\mathcal{F}}^{(3)}$ described in Section 3.3 look as follows:

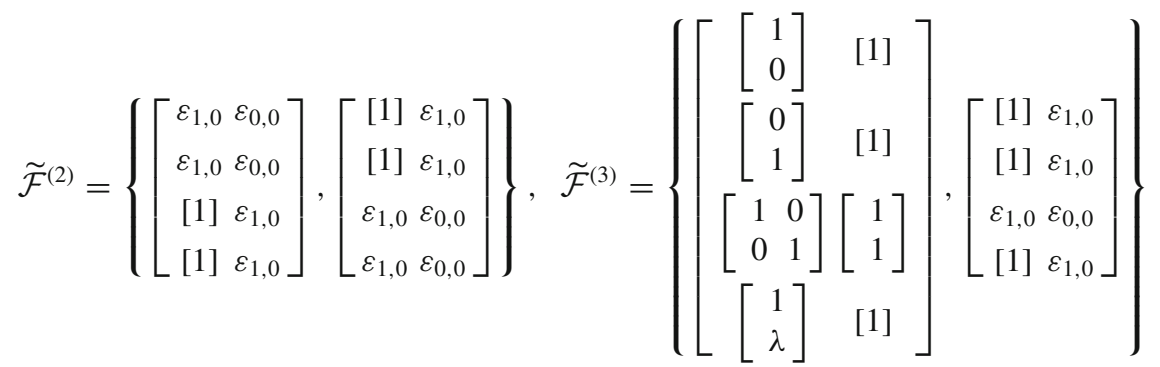

where $\varepsilon_{x, y} \in \mathbb{M}_{x \times y}(R)$ denotes the trivial matrix, for $x=0$ or $y=0$. We take the bimodule $B^{(4)}$ from [9, Table 4.7]:

$$
B^{(4)}=\left[\begin{array}{ll}
{\left[\begin{array}{lll}
0 & 1 & 0 \\
\xi & 0 & 0 \\
0 & 0 & 1 \\
1 & 0 & 0
\end{array}\right]\left[\begin{array}{ll}
0 & 0 \\
1 & 0 \\
0 & 1
\end{array}\right]} \\
{\left[\begin{array}{lll}
1 & 0 & 0 \\
0 & 1 & 0 \\
1 & 0 & 0 \\
0 & 0 & 1
\end{array}\right]\left[\begin{array}{ll}
0 & 0 \\
1 & 0 \\
0 & 1
\end{array}\right]} \\
{\left[\begin{array}{lll}
1 & 0 & 0 \\
0 & 1 & 0 \\
0 & 0 & 1 \\
0 & 0 & 1
\end{array}\right]\left[\begin{array}{ll}
1 & 0 \\
1 & 0 \\
0 & 1
\end{array}\right]} \\
{\left[\begin{array}{lll}
1 & 0 & 0 \\
\lambda & 0 & 0 \\
0 & 1 & 0 \\
0 & 0 & 1
\end{array}\right]\left[\begin{array}{ll}
1 & 0 \\
0 & 1 \\
0 & \lambda
\end{array}\right]}
\end{array}\right] .
$$

Next applying the description of universal extension given in Theorem 4.1, expressed via the interpretation of the extension group in terms of derivation modules 
(see Lemma 4.2), we compute inductively the consecutive bimodule universal extensions appearing in $(*)$ :

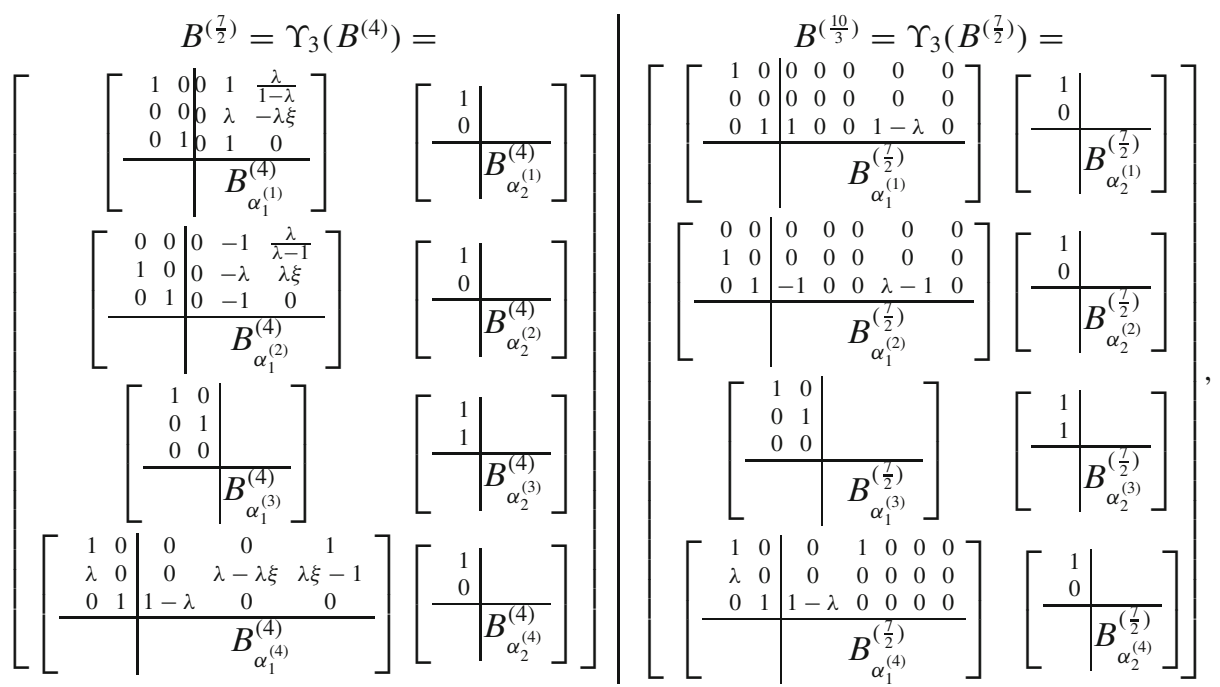

and finally we obtain $B^{\left(\frac{18}{7}\right)}=\Upsilon_{2}\left(B^{\left(\frac{10}{3}\right)}\right)=$

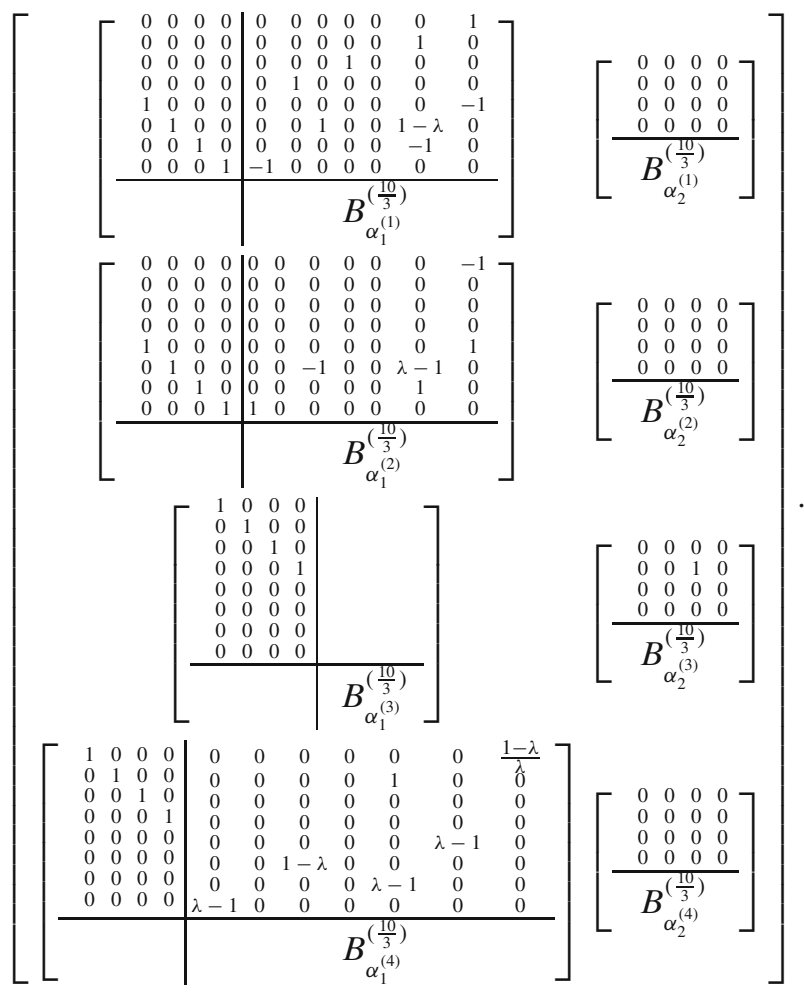

(The empty spaces in the matrices above always mean the zero blocks). Note that $\operatorname{rk}_{R}\left(\operatorname{Ext}_{R \otimes \Lambda}^{1}\left(B^{(4)}, \widetilde{F}\right)\right)=1=\operatorname{rk}_{R}\left(\operatorname{Ext}_{R \otimes \Lambda}^{1}\left(B^{\left(\frac{7}{2}\right)}, \widetilde{F}\right)\right)$, for each $\widetilde{F} \in \widetilde{\mathcal{F}}^{(3)}$, and 
$\operatorname{rk}_{R}\left(\operatorname{Ext}_{R \otimes \Lambda}^{1}\left(B^{\left(\frac{10}{3}\right)}, \widetilde{F}\right)\right)=4$, for each $\widetilde{F} \in \widetilde{\mathcal{F}}^{(2)}$. To compute the respective ranks and bases we use the formulas for derivation modules given in Lemma 4.8 and apply a more sophisticated version of the algorithm creating Smith diagonal form for the $R$-matrices from Section 4.8 (cf. [20]).

The details concerning linear algebra calculus realizing all the $R \otimes_{k} \Lambda$-module constructions, which appear in our method, will be presented in forthcoming publication.

Remark Independently on the theoretical considerations, all the bimodules computed above have passed detailed tests in order to make sure we did not make any miscalculations during the process of their construction. In particular, we checked that the specializations $B^{(q)}(a, l):=R /(\xi-a)^{l} \otimes_{R} B^{(q)}$, for $(a, l) \in(k \backslash\{0,1, \lambda\}) \times$ $\mathbb{N}_{+}$even for $(a, l) \in k \times \mathbb{N}_{+}$, and $q \in\left\{\frac{18}{7}, \frac{10}{3}, \frac{7}{2}\right\}$ (cf. Section 2.1) indeed:

- have appropriate dimension vectors and satisfy relations,

- are indecomposable (their endomorphism algebras turned out to be local),

- $\quad$ are Hom-orthogonal (i.e. for fixed slope $q, \operatorname{Hom}_{\Lambda}\left(B^{(q)}(a, l), B^{(q)}\left(a^{\prime}, l^{\prime}\right)\right)=0$ for any $a \neq a^{\prime}$ and $\left.l, l^{\prime} \geq 1\right)$.

Open Access This article is distributed under the terms of the Creative Commons Attribution License which permits any use, distribution, and reproduction in any medium, provided the original author(s) and the source are credited.

\section{References}

1. Anderson, F.W., Fuller, K.R.: Rings and categories of modules. In: Graduate Texts in Math., vol. 13. Springer, New York (1992)

2. Assem, I., Simson, D., Skowroński, A.: Elements of Representation Theory of Associative Algebras, vol. 1. Techniques of Representation Theory, London Math. Soc. Student Texts 65. Cambridge University Press, Cambridge (2006)

3. Cartan, H., Eilenberg, S.: Homological algebra. In: Princeton Mathematical Series, vol. 19. Princeton University Press, Princeton (1956)

4. Crawley-Boevey, W.W.: Tame algeras and generic modules. Proc. Lond. Math. Soc. 63(3), 241265 (1991)

5. Dowbor, P., Mróz, A.: The multiplicity problem for indecomposable decompositions of modules over domestic canonical algebras. Colloq. Math. 111(2), 221-282 (2008)

6. Dowbor, P., Mróz, A.: On a separation of orbits in the module variety for domestic canonical algebras. Colloq. Math. 111(2), 283-295 (2008)

7. Dowbor, P., Mróz, A.: On a separation of orbits in the module variety for special biserial algebras. J. Pure Appl. Algebra 213(9), 1804-1815 (2009)

8. Dowbor, P., Meltzer, H., Mróz, A.: An algorithm for the construction of exceptional modules over tubular canonical algebras. J. Algebra 323(10), 2710-2734 (2010)

9. Dowbor, P., Meltzer, H., Mróz, A.: Parametrizations for integral slope homogeneous modules over tubular canonical algebras. Algebr. Represent. Theory (2013). doi:10.1007/s10468-012-9386-7

10. Gantmacher, F.R.: Matrix Theory. Chelsea, New York (1959)

11. Geigle, W., Lenzing, H.: A class of weighted projective curves arising in representation theory of finite dimensional algebras. In: Singularities, Representations of Algebras, and Vector Bundles. Lecture Notes in Mathematics, vol. 1273, pp. 265-297. Springer (1987)

12. Happel, D.: Triangulated categories in the representation theory of finite dimensional algebras. In: London Math. Soc. Lecture Notes Series, vol. 119 (1988)

13. Kędzierski, D., Meltzer, H.: Indecomposable represenations for extended Dynkin quivers of type $\tilde{\mathbb{E}}_{8}$. Colloq. Math. 111(1), 95-116 (2011) 
14. Lenzing, H., Meltzer, H.: Sheaves on a weighted projective line of genus one and representations of a tubular algebra. In: Representations of Algebras, Sixth International Conference, Ottawa 1992. CMS Conf. Proc., vol. 14, pp. 317-337 (1993)

15. Meltzer, H.: Tubular mutations. Colloq. Math. 74(2), 267-274 (1997)

16. Meltzer, H.: Exceptional vector bundles, tilting sheves and tilting complexes for weighted projective lines. Memoirs of AMS, vol. 171, no. 808 (2004)

17. Meltzer, H.: Exceptional modules for tubular canonical algebras. Algebr. Represent. Theor. 10, 481-496 (2007)

18. Simson, D., Skowroński, A.: Elements of the Representation Theory of Associative Algebras, vol. 3. Representation-Infinite Tilted Algebras, London Math. Soc. Student Texts 71. Cambridge University Press, Cambridge (2007)

19. Ringel, C.M.: Tame algebras and integral quadratic forms. In: Springer Lecture Notes in Mathematics, vol. 1099 (1984)

20. Smith, H.J.S.: On systems of linear indeterminate equations and congruences. In: Collected Math. Papers, vol. 1, pp. 367-409. Chelsea (1979) 